\title{
Motion and Force Transmission of a Flexible Instrument
}

Inside a Curved Endoscope

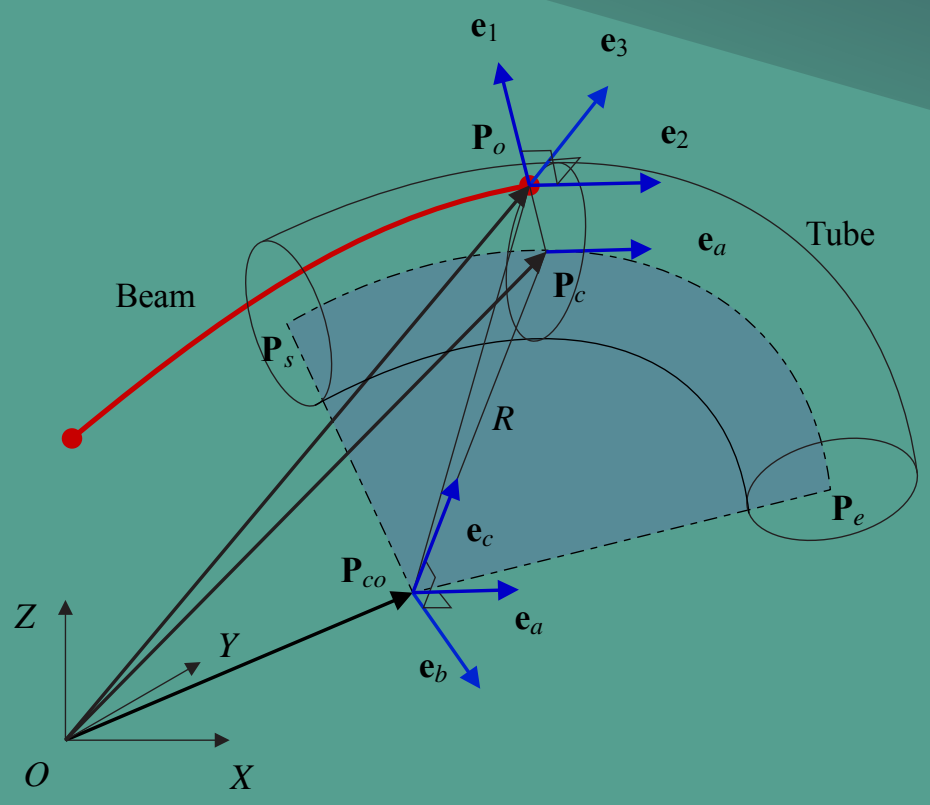

Jitendra Prasad Khatait 



\section{MOTION AND FORCE TRANSMISSION OF A FLEXIBLE INSTRUMENT INSIDE A CURVED ENDOSCOPE}




\section{Composition of the Graduation Committee:}

Chairman and Secretary:

Prof. Dr. F. Eising University of Twente

Promoter and Assistant Promoters:

Prof. Dr. Ir. J.L. Herder

University of Twente

Dr. Ir. D.M. Brouwer

University of Twente

Dr. Ir. R.G.K.M. Aarts

University of Twente

\section{Members:}

Prof. Dr. S. Mukherjee

Indian Institute of Technology Delhi

Prof. Dr. Ir. P. Breedveld

Delft University of Technology

Prof. Dr. Ir. S. Stramigioli

University of Twente

Prof. Dr. Ir. F.J.A.M. van Houten

University of Twente

This research is funded with the TeleFLEX project, number PID07038, by the Dutch Department of Economic Affairs, Agriculture and Innovation and the Province of Overijssel, within the Pieken in de Delta (PIDON) initiative.

Cover Design: The background includes a photo of the experimental set-up. The figure on the front cover shows the contact triad at the interacting node of the beam inside a circular tube. The figure on the back cover is a painting from my little daughter, Bhairavi.

Title: Motion and Force Transmission of a Flexible Instrument Inside a Curved Endoscope

Author: Jitendra Prasad Khatait

ISBN: $\quad$ 978-90-365-0010-4

DOI: $\quad$ http://dx.doi.org/10.3990/1.9789036500104

Copyright (C) 2013 by Jitendra Prasad Khatait, Enschede, The Netherlands. All rights reserved. No part of this publication may be reproduced by print, photocopy or any other means without the prior written permission from the copyright owner.

Printed by Ipskamp Drukkers, Enschede, The Netherlands. 


\section{MOTION AND FORCE TRANSMISSION OF A FLEXIBLE INSTRUMENT INSIDE A CURVED ENDOSCOPE}

\section{DISSERTATION}

to obtain

the degree of doctor at the University of Twente, on the authority of the rector magnificus,

Prof. Dr. H. Brinksma,

on account of the decision of the graduation committee,

to be publicly defended

on Thursday 5th of September 2013 at 14.45 hours

by

Jitendra Prasad Khatait

born on 21 September 1977

in Narayan Garh, West Champaran, INDIA 
This thesis has been approved by:

Promoter: Prof. Dr. Ir. J.L. Herder

Assistant Promoters: Dr. Ir. D.M. Brouwer

Dr. Ir. R.G.K.M. Aarts 
Dedicated to my parents 



\section{ConTENTS}

Contents

vii

1 Introduction 1

1.1 Background . . . . . . . . . . . . . . . 1

1.1.1 Minimally Invasive and Robotic Surgery _... . . . 2

1.1.2 Flexible Endoscopic Surgery . . . . . . . . . . . . . . 4

1.2 Motivation . . . . . . . . . . . . . . . . 7

1.3 Research Objective . . . . . . . . . . . . . . . 8

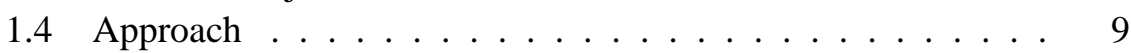

1.5 Contributions of the Thesis . . . . . . . . . . . 10

1.6 Outline of the Thesis . . . . . . . . . . . . . . 11

2 2-D Modelling of a Flexible Instrument 13

2.1 Introduction . . . . . . . . . . . . . . . . 14

2.2 Modelling of a Surgical Instrument . . . . . . . . . . . . 16

2.2.1 Finite Element Model of the Surgical Instrument . . . 17

2.2.2 Model of a Curved Endoscope . . . . . . . . . . . 20

2.2.3 Interaction of Beam with Inner Wall of Tube . . . . . 23

2.2 .4 Discussion . . . . . . . . . . . . . . . 27

2.3 Simulation . . . . . . . . . . . . . . . . 27

2.3.1 Simulation of Flexible Beam Insertion . . . . . . . . . 29

2.3.2 Simulation of Fine Manipulation . . . . . . . . . . . 33

2.4 Discussion . . . . . . . . . . . . . . . . 36

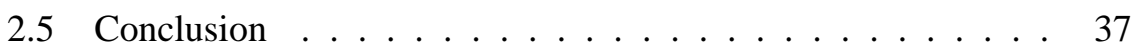

3 Design of an Experimental Set-up 39

3.1 Introduction . . . . . . . . . . . . . . 40 
3.2 Design Objective . . . . . . . . . . . . . . . . . . . 41

3.2 .1 Design Requirements . . . . . . . . . . . . . . . . 42

3.2 .2 Design Specifications . . . . . . . . . . . . . . . 43

3.3 Design of the Experimental Set-up . . . . . . . . . . . . . 44

3.3.1 Design of the AM . . . . . . . . . . . . . . . 44

3.3.2 Design of the FSM . . . . . . . . . . . . . . 48

3.3.3 Design of the T3M . . . . . . . . . . . . . 52

3.3.4 Real-time Measurement System . . . . . . . . . . 54

3.3.5 Discussion . . . . . . . . . . . . 55

3.4 Design Evaluation . . . . . . . . . . . . . . . . 56

3.4.1 Evaluation of the AM and the T3M . . . . . . 56

3.4 .2 Evaluation of the FSM . . . . . . . . . . . 63

3.5 Discussion . . . . . . . . . . . . . . . . . . 68

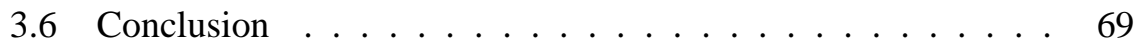

4 3-D Modelling of a Surgical Instrument 71

4.1 Introduction . . . . . . . . . . . . . . . . 72

4.2 Modelling of a Surgical Instrument . . . . . . . . . . . . 74

4.2.1 Instrument as Beam Elements . . . . . . . . . . . . 74

4.2.2 An Endoscope as a Rigid Curved Tube . . . . . . . 79

4.2.3 Contact Force Model . . . . . . . . . . . . . . . . 82

4.3 Simulation . . . . . . . . . . . . . . . . 84

4.3.1 Fine Manipulation in Translation . . . . . . . . . . . . . 85

4.3.2 Fine Manipulation in Rotation . . . . . . . . . . . 91

4.3.3 Discussion . . . . . . . . . . . . . . . 95

4.4 Experimental Set-up and Validation . . . . . . . . . . 95

4.4.1 Motion and Force Transmission During Translation . . 96

4.4.2 Motion Transmission During Rotation . . . . . . . . 100

4.5 Discussion . . . . . . . . . . . . . . . . . . 102

4.6 Conclusion . . . . . . . . . . . . . . . 103

5 Improved Force Transmission $\quad 105$

5.1 Introduction . . . . . . . . . . . . . . 106

5.2 Methods . . . . . . . . . . . . . . . . 107

5.2.1 Analytical Formulation . . . . . . . . . . . . . 108

5.2.2 Flexible Multibody Model . . . . . . . . . . . . . 114

5.2 .3 Experiments . . . . . . . . . . . . . . 117

5.3 Results . . . . . . . . . . . . . . . . . . . . . . . 119

5.3.1 Simulation Results . . . . . . . . . . . . . . . . . 119 
5.3.2 Experimental Results . . . . . . . . . . . . . . . . 121

5.4 Discussion . . . . . . . . . . . . . . . . . . . . . 134

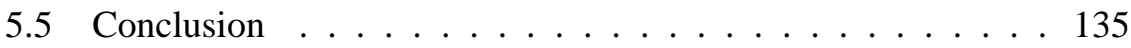

6 Conclusions and Recommendations 137

6.1 Conclusions . . . . . . . . . . . . . . . . . 137

6.1.1 2-D Flexible Multibody Modelling . . . . . . . . . 138

6.1.2 Design and Evaluation of an Experimental Set-up . . . 138

6.1.3 3-D Modelling and Experimental Validation . . . . . . 139

6.1 .4 Improved Force Transmission . . . . . . . . . . . 140

6.1.5 Overall Conclusion . . . . . . . . . . . . . . . . . 140

6.2 Recommendations . . . . . . . . . . . . . . . . . . . . 142

6.2.1 Modelling and Simulation . . . . . . . . . . . 142

6.2.2 Design of a Flexible Instrument . . . . . . . . . . . 142

6.2.3 Improvement in the Experimental set-up . . . . . . . . 143

6.2 .4 Other Applications ． . . . . . . . . . . . . . . . . 144

$\begin{array}{ll}\text { References } & 145\end{array}$

A The Planar Beam Element 153

$\begin{array}{ll}\text { Summary } & 155\end{array}$

$\begin{array}{ll}\text { Acknowledgements } & 157\end{array}$

About the Author 159 



\section{INTRODUCTION}

This chapter introduces the motivation and the objective of this research. A brief introduction on the technological advancement in modern surgery is presented, particularly how the minimally invasive surgery has enhanced the quality of the surgical outcome. The chapter highlights the technical challenges and the gaps towards achieving the best surgical care to the patient while minimizing the trauma during and after the surgical intervention. The research objective is defined and the individual research goals are identified. The methodology is outlined to achieve the research objective. Finally, the chapter highlights the contributions of this research and outlines the structure of the thesis.

\subsection{Background}

Modern surgery has progressed rapidly with advances in medical science and technology. Three key developments in medical science-the control of bleeding, pain, and infection-have allowed surgeons to treat any part of the body by making a large incision to access the surgical site $[16,51]$. They have a large dexterous space and they can make all the six degrees-of-freedom movements required to complete the surgical procedure. The surgical procedure requires several layers of incision and dissection of healthy tissues. However, unnecessary damage and trauma to healthy tissue is clearly undesirable and can lead to post-operative pain, lengthened hospital stays, and sometimes to serious complications. The excessive mutilation of the healthy tissues occurs mostly to gain access to the area to perform the intended procedure rather than 


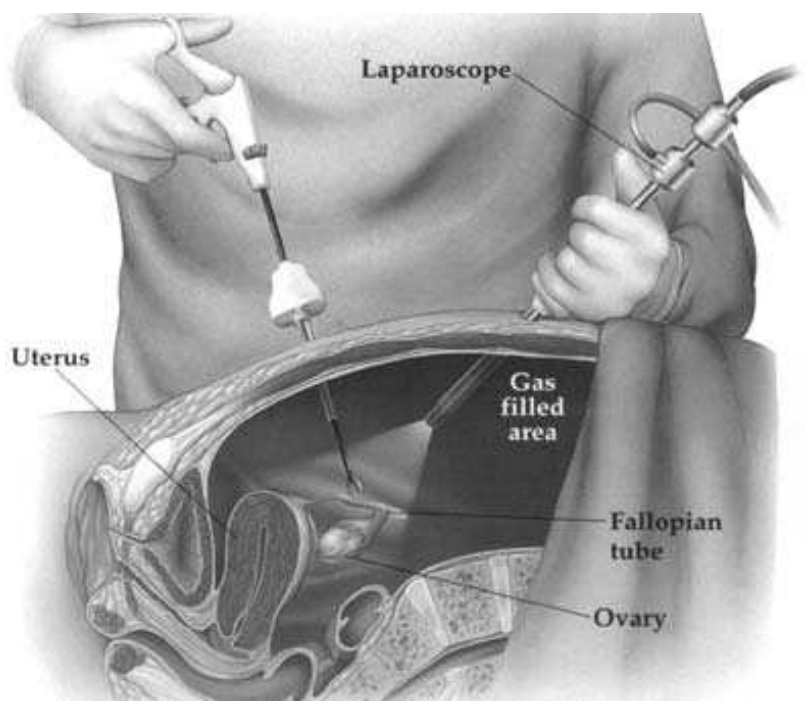

Fig. 1.1: Laparoscopic surgery using rigid instruments where internal organs are accessed through small incisions in the abdominal wall [33]

the procedure itself $[6,34]$. The advent of laparoscopic techniques-in which rigid instruments are inserted through a small incision in the abdominal wall to access the interior of the peritoneal cavity-to perform surgical procedures has revolutionized the surgery in the $21^{s t}$ century [50]. The laparoscopic procedures have greatly reduced the excessive damage to the healthy tissues.

\subsubsection{Minimally Invasive and Robotic Surgery}

\section{Minimally Invasive Surgery}

Minimally invasive surgery (MIS) or a keyhole surgery is a modern technique in which the surgical site is accessed via small incisions on the skin unlike the larger incisions needed in the conventional surgical procedure [8]. A small cut in the skin allow surgeons to introduce a harmless gas, such as carbon dioxide as the insufflation gas, into the body cavity for the creation of the pneumoperitoneum; and, thus, it provides a large working space. A limited number of round cannulas (trocars) are inserted through the small incisions. Long thin instruments are introduced through the trocars to perform the necessary surgical procedures inside the body of the patient (Fig. 1.1). A small camera is also introduced through one of the trocars to observe the actions from outside. MIS can be applied to different parts of the body. Laparoscopy refers to the application of the technique to the abdomen. 
The introduction of laparoscopic procedures has benefited the patients immensely by greatly reducing the risk of wound infections, reduced postoperative pain, an earlier return to normal function, and improved patient satisfaction $[8,50]$. With the technical improvements and the developments of laparoscopic instruments, more and more minimally invasive interventions have replaced the conventional open surgery.

However, laparoscopic technique has introduced several challenges by changing the way the surgeon observes and manipulates the tissue. Now, the surgeon has to manipulate the tissue via laparoscopic instruments, inserted through small incisions, with limited freedom of movement. The dexterity of the surgeon is compromised. The surgeon has no direct contact with the tissue. There is a loss of haptic feedback (force and tactile) and natural hand-eye coordination. Nonetheless, there are clear and proven benefits to the patients. The limitations and challenges are mainly technological and can be overcome with technological advancement.

\section{Robotic Surgery}

The motivation to develop surgical robots is rooted in the desire to overcome the limitations of current laparoscopic technologies and to make the clear benefits of MIS more widely available. Robotic systems have the potential to improve dexterity, restore proper hand-eye coordination and enhance ergonomics of the working position, and improve visualization [32].

Dexterity is enhanced by decoupling the surgeon's and instrument's workspace. The surgeon has a dexterous workspace at the surgeon's console while manipulating the remote surgical instruments via a computer (Fig. 1.2). Instruments with increased degrees of freedom greatly improve the surgeon's ability to manipulate instruments and tissues [34].

In addition, these robotic systems can scale movements so that long movements of the control grips can be transformed into micro-motions inside the patient. These systems are designed so that the surgeon's hand tremor can be compensated for at the end-effector motion. Another important advantage is the restoration of effective hand-eye coordination and an ergonomic working position. These robotic systems eliminate the fulcrum effect, making instrument manipulation more intuitive. There is remarkable improvement in the vision afforded by these systems. The three-dimensional view with depth perception is a marked improvement over the conventional laparoscopic camera views. However, these systems still have many limitations-efficacy, cost, size, lack of compatible instruments and equipments, etc. 


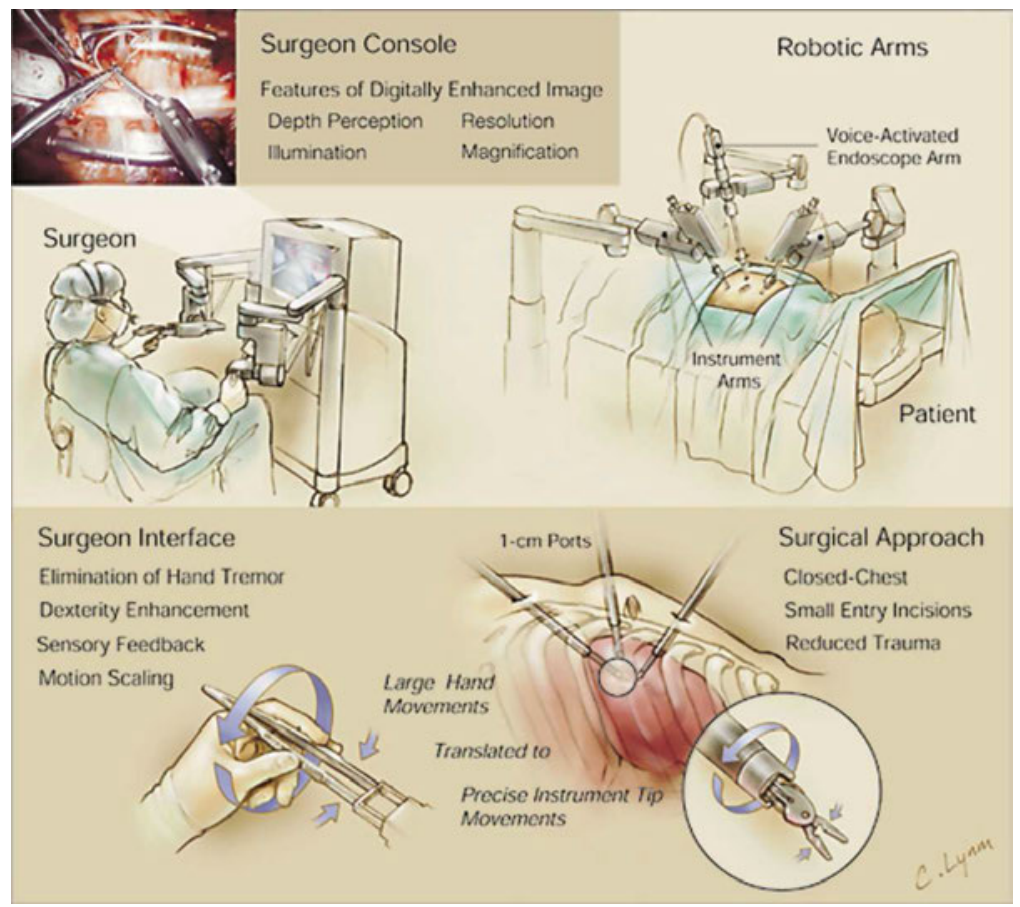

Fig. 1.2: Surgery with a robotic system where a surgeon controls the instrument tip movements remotely from the surgeon console [34]

\subsubsection{Flexible Endoscopic Surgery}

Endoscopy is a major advance in the treatment of gastrointestinal (GI) and pulmonary diseases. The word endoscopy [48] is of Greek origin and literally means observing within or looking inside. The original concept of endoscopic procedures was driven by the need to evaluate the inner surface (the lumen) of internal organs [4]. Endoscopy is often performed in an outpatient setting, and is well-tolerated by patients, allowing doctors to detect ulcers, cancers, polyps, internal bleeding and other disorders of the GI tract and pulmonary tree (Fig. 1.3). Endoscopic techniques are safe, cost-effective and have a very low rate of complications.

An endoscope is a long and thin flexible tube consisting of several channels for visualization, light transmission, suction and irrigation. Some more access channels are provided for flexible instruments to perform various surgical interventions (Fig. 1.4). The flexible instrument is inserted through the access channel of the endoscope; and the instrument tip is manipulated from proximal end of the instrument. 


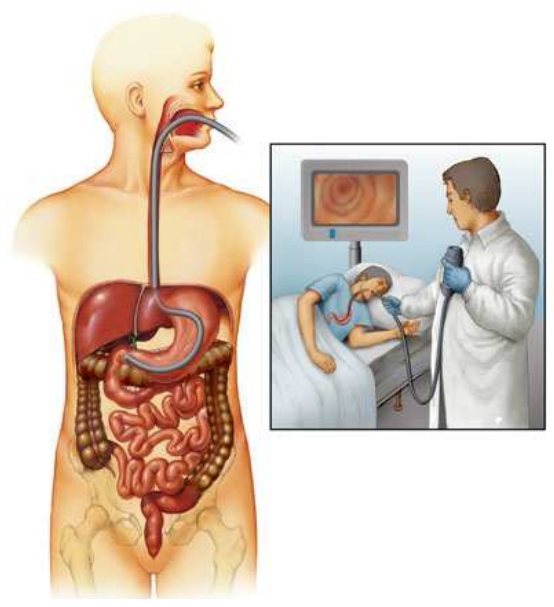

Fig. 1.3: Endoscopy used to examine upper digestive system [39]

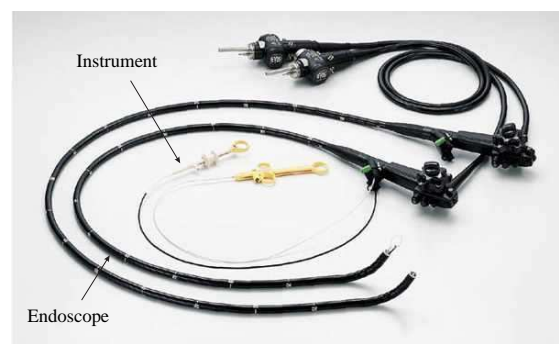

Fig. 1.4: Flexible endoscope with instrument [49]

\section{Emergence of NOTES and SILS procedures}

Endoscopy was initially used for mainly diagnosis purposes. Later, therapeutic procedures were also performed through flexible endoscopy, such as repairing bleeding ulcers and veins, removing non-cancerous growth (polyps), or early stage cancerous tumours, endoscopic submucosal dissection, etc. Flexible endoscopy is becoming increasingly invasive.

On the other hand, conventional surgery is becoming less invasive. It has already moved from open surgery to MIS. Increasingly, more surgical procedures are carried out through MIS. The list is still growing at a pace consistent with technological improvements and surgeon's technical skills.

Considering these two trends in medical science, it is evident that surgery and endoscopy will eventually work closely together (Fig. 1.5). Technological developments in this direction are already taking place and a plethora of research among different scientific communities is reported in scientific journals [57]. This leads to two very topical and challenging research directions both in the surgical and the engineering communities, namely Natural Orifice Transluminal Endoscopic Surgery (NOTES) and Single Incision Laparoscopic Surgery (SILS) [7,57].

NOTES and SILS definitely show a paradigm shift in the area of endoscopic surgery. Several researchers have already started incorporating advance features to the current endoscopic systems so that complex surgical manipulations can be accomplished $[58,59]$. 


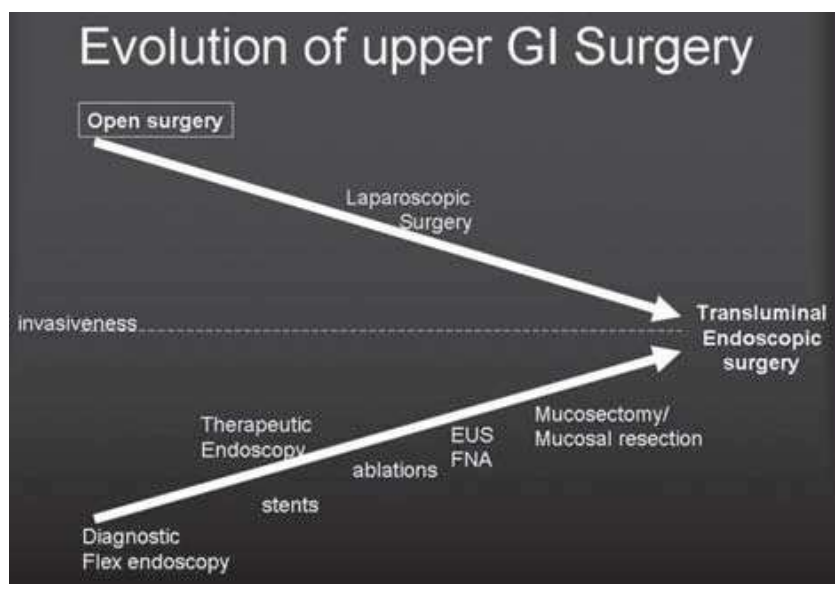

Fig. 1.5: Merging of surgery and therapeutic endoscopy [57]

However, several important challenges, including the safety of these approaches, must be resolved before the new techniques are widely introduced into clinical use. Moreover, currently available flexible endoscopes and instruments are inadequate for performing complex surgical procedures. The next generation endoscopic systems should include advance features like shapelocking ability to position and then stiffen the endoscopic platform to allow stable and robust exposure and retraction, adequate number and size of access channels for different kinds of instruments. There is a need to develop true flexible surgical tools for aggressive retraction of organs, tissue approximation and deep closure [59].

There are several developments being investigated among the research communities to achieve better endoscopic systems. Several leading medical device manufacturers-such as Boston Scientific, USGI Medical, Olympus, and Karl Storz-are already developing new generation flexible endoscopic operating systems that promise to meet the needs of complex surgery (Fig. 1.6) [24,59]. Though these endoscopic systems have started to incorporate tools that can enable NOTES-like procedures, they are still difficult and cumbersome to use. They face limitations similar to those already encountered with normal laparoscopic procedures. Integrating robotic technology with the flexible endoscopic system is the logical step in developing fully integrated solution. Nonetheless, the development of such systems opens up an immense opportunity for endo/transluminal endoscopic surgery. A better platform, tools and techniques for carrying out endoscopic surgery can be of great help to surgeons. 


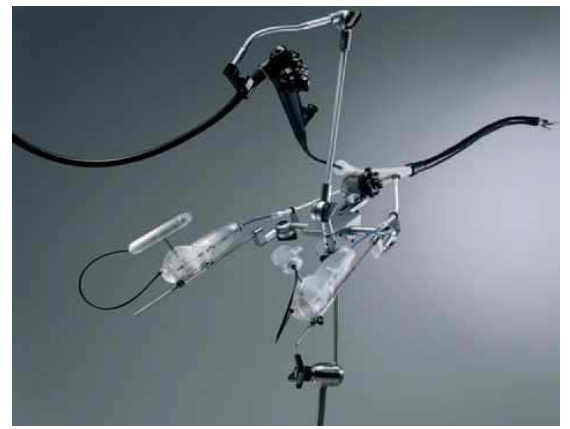

(a) DDES

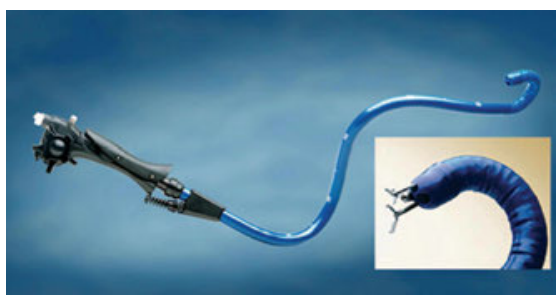

(b) EOS

Fig. 1.6: (a) Direct Drive Endoscopic System prototype (DDES, Boston Scientific) [24]. (b) Endosurgical Operating System (EOS, USGI Medical) [59].

\subsection{Motivation}

Development of a fully integrated system — where the surgeon manipulates the surgical instruments from his/her master console and the surgical instrument navigates through the lumen of the body and completes the surgical intervention with precision, accuracy and safety at the command of the surgeon-is the next step towards accomplishing complex surgical procedures with minimum trauma and damage to the healthy tissues.

A flexible endoscopic system has well understood advantages towards navigating and accessing the interior organs in comparison to the rigid instruments. A flexible instrument is guided through the endoscope. Integrating the instrument with a master controller, the instrument tip can be controlled more intuitively and efficiently. A master-slave concept of the type shown in Fig. 1.7 can solve many of the technical challenges encountered with such integrated endoscopic systems.

The flexible instruments have to perform more complex surgical manipulations like needle steering, suturing, cutting, tissue manipulation, etc. The instrument tip needs an accurate positioning and orientation. The motion of the tip should be smooth and free from unwanted jitters and sudden movements. The tip also needs to apply requisite amount of force to complete the intended task safely. Use of excessive force can damage the healthy tissues or organs and can lead to undesirable outcomes. Therefore, the motion and force fidelity of the instrument is of paramount importance for the safety of the patient as well as for the overall performance of the system. 


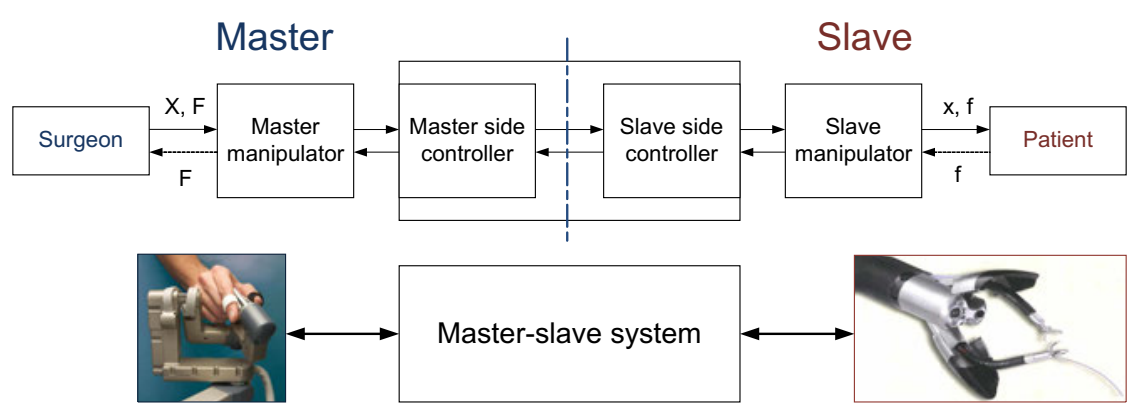

Fig. 1.7: Master-slave concept for robotic surgery using flexible endoscopic system

Control of the flexible instrument tip is very challenging. The motion is not as smooth as in the case of rigid instruments. The surgeon may need to retract and readjust for the overshoot. The tip motion is accompanied with sudden movements. These kinds of movements, if very large, make the instrument unusable and unsafe. These undesirable motion characteristics of the instrument can be compensated to some extent by a controller if the characteristic behaviour of the instrument is known. However, a conventional controller cannot accomplish accurate manipulation with an instrument, if the motion is hampered by a large static friction force. Proper strategies have to be investigated to improve the force transmission.

The motion and force transmission of a flexible instrument inside the access channel of the endoscope or endoscopic platform is governed by the mechanical properties of the instrument and the endoscope, contact parameters, and the overall shape of the endoscope inside the body. The shape of the endoscope is not fixed and depends on the surgical site. Friction and the finite stiffness of the instrument limit the motion and force transmission. A prior knowledge of the static and dynamic behaviour of the instrument in the presence of friction under varying geometry of the endoscope will help in an accurate and precise control of motion and force delivery at the instrument tip.

\subsection{Research Objective}

A thorough understanding of flexible instrument behaviour inside the access channel of an endoscope is needed for its successful and safe integration in a master-slave robotic system. This brings more transparency and confidence in using flexible devices for clinical usage. However, the fundamental research 
towards characterizing flexible instrument behaviour inside an endoscope is missing. Therefore, the objective of this research is stated as:

Characterization of static and dynamic behaviour of a flexible surgical instrument inside a curved rigid tube-both for translation and rotation motion input; develop a 2-D and 3-D flexible multibody model; design a dedicated experimental set-up for the actuation and measurement of motion and interaction forces; and to derive strategies to improve motion and force fidelity of the instrument.

\subsection{Approach}

In order to achieve the research objective, a flexible multibody model is developed for the instrument. A proper model for the curved tube is proposed to define the shape of the tube. A contact model is defined, which includes the wall stiffness, damping and the friction between the contacting surfaces.

A computer program, SPACAR [21], is used for the modelling and simulation of the flexible surgical instrument inside a curved tube. SPACAR is a modelling and simulation tool based on finite element method (FEM) for multibody dynamic analysis of planar and spatial mechanisms and manipulators with flexible links.

The modelling and simulation has been developed in two stages. Firstly, a 2-D model is developed which is used to study the sliding behaviour of the instrument in a planar tube. The simulation results are compared with the analytical results. Secondly, a 3-D model is developed to study the behaviour of the instrument both in translation and rotation. A curved rigid tube is defined in a 3-D space. The simulation results are compared with the analytical and the experimental results.

An experimental set-up is designed based on the technical specifications required to validate the model and to characterize the behaviour of a range of instruments. It can provide the required actuation, measure the input and output motions, and measure the forces due to the interaction. The set-up includes a measurement and a data acquisition system, i.e., real-time PC-based system. The set-up is evaluated against the required technical specifications.

The set-up is used to validate the developed model for different motion inputs. The characteristic behaviour of a flexible instrument is studied for translation and rotation motion input using the developed model and the experimental set-up. The motion and force transmission of the instrument is investigated 
Chapter 1. Introduction

for unloaded and loaded case. Loading is applied at the distal end through a spring load.

A strategy to improve the force transmission along the axial direction is proposed based on the initial modelling and experimental results. An analytical model is developed to study the effect of the combined motion on alleviating friction and, thus, improving the force transmission. The developed model and the experimental set-up are used to verify the analytical results.

The methodology adopted to achieve the research objective is summarized as:

- Develop a 2-D flexible multibody model of an instrument to study its sliding behaviour inside a curved rigid tube

- Design and evaluation of an experimental set-up for the validation of the developed flexible multibody model and characterization of flexible instruments for surgical application

- Set up a 3-D flexible multibody model of a surgical instrument to study its translational and rotational behavior in a 3-D environment, and validate the model with the experimental results

- Improve the force transmission of a flexible instrument along the axial direction through a curved rigid tube by combining the translation input motion with rotational motion

\subsection{Contributions of the Thesis}

The outcomes of this research are presented to various international conferences and submitted to international journals. The scientific outputs as peerreviewed journal articles and conference papers are:

\section{Journal articles}

- J.P. Khatait, D.M. Brouwer, R.G.K.M. Aarts, J.L. Herder (2013). Improved force transmission of a flexible surgical instrument by combined input motion, Precision Engineering, Draft submitted.

- J.P. Khatait, D.M. Brouwer, J.P. Meijaard, R.G.K.M. Aarts, J.L. Herder (2013). Flexible multibody modelling of a surgical instrument inside a curved endoscope, Journal of Computational and Nonlinear Dynamics, Under review. 
- J.P. Khatait, D.M. Brouwer, H.M.J.R. Soemers, R.G.K.M. Aarts, J.L. Herder (2013). Design of an experimental set-up to study the behavior of a flexible surgical instrument inside an endoscope, Journal of Medical Devices, Volume 7, Issue 3, 031004(2013)(12 pages), DOI: 10.1115/1.4024660.

- J.P. Khatait, D.M. Brouwer, R.G.K.M. Aarts, J.L. Herder (2012). Modeling of a flexible instrument to study its sliding behavior inside a curved endoscope, Journal of Computational and Nonlinear Dynamics, Volume 8, Issue 3, 031002(2012)(10 pages), DOI: 10.1115/1.4007539.

\section{Conference papers}

- J.P. Khatait, D.M. Brouwer, J.P. Meijaard, R.G.K.M. Aarts, J.L. Herder (2012). 3-D multibody modeling of a flexible surgical instrument inside an endoscope, ASME 2012 International Mechanical Engineering Congress $\mathcal{E}$ Exposition, November 9-15, 2012, Houston, Texas, USA.

- J.P. Khatait, D.M. Brouwer, R.G.K.M. Aarts, J.L. Herder (2012). Test Set-up to Study the Behavior of a Flexible Instrument in a Bent Tube, ASME 2012 Design of Medical Devices Conference (DMD2012), April 10-12, 2012, Minneapolis, Minnesota, USA.

- J.P. Khatait, M. Krijnen, J.P. Meijaard, R.G.K.M. Aarts, D.M. Brouwer, J.L. Herder (2011). Modelling and Simulation of a Flexible Endoscopic Surgical Instrument in a Tube, ASME 2011 International Mechanical Engineering Congress $\mathcal{E}$ Exposition, Volume 2, Pages 557-566, ISBN: 978-0-7918-5488-4, DOI:10.1115/IMECE2011-65189.

\subsection{Outline of the Thesis}

The journal articles constitute the main chapters of the thesis. The topics and contents of the journal articles are conceived to maintain the flow and the structure of the research and, therefore, of the thesis as well. The journal articles are self-contained and can be read independently. As they are the part of the main research objective, they share the introduction and motivation; but they also fulfill their individual objectives. The contents of the individual chapters are described below.

Chapter 2 describes the 2-D modelling of the flexible instrument inside a curved rigid tube. The flexible instrument, the endoscope, and the interaction 
between the instrument and the endoscope are appropriately defined. Simulations are performed for the insertion and fine manipulation of the instrument inside the endoscope. The sliding behaviour of the instrument is characterised under variation of the coefficient of friction and the bending stiffness of the instrument. The stick-slip behaviour and the motion hysteresis is investigated.

Chapter 3 covers the design and evaluation of the experimental set-up to study the behaviour of a flexible instrument inside an endoscope. The design specifications are established based on the requirements. The concepts and the detailed design are discussed for the various modules. The performance is evaluated against the stated specifications.

Chapter 4 contains the 3-D modelling of the flexible surgical instrument inside a curved endoscope. A general 3-D model is developed to incorporate all the mechanical properties of the instrument so that both translational and rotational behaviour can be studied in the presence of friction. Various simulations are performed for the axially-loaded and no-load cases. The simulation results are compared with the analytical capstan equation. Various experiments are performed using the designed set-up and the results are used to validate the developed model.

Chapter 5 describes the strategy to improve force transmission of a flexible instrument along the axial direction by combining the translation motion input with the rotation input. An analytical formula is derived and the effect of the combined motion is studied. It is shown that friction force along the axial direction can be reduced by combining the translation motion input with the rotation input. The developed 3-D model is used to perform various simulations and the results are compared with the analytical results. Several experiments are performed with constant translation motion input combined with constant and sinusoidal rotational motion input. Input and output forces are measured and the results are presented.

Chapter 6 concludes the research findings. The individual contributions of each chapter are discussed with respect to the overall research objective. It also highlights the recommendations which can be pursued further to achieve the underlined research objective. 
Modelling of A FleXIBLE Instrument to StUdy ITS SLIDING Behaviour Inside a Curved ENDOSCOPE

Flexible instruments are increasingly used to carry out surgical procedures. The instrument tip is remotely controlled by the surgeon. The flexibility of the instrument and the friction inside the curved endoscope jeopardize the control of the instrument tip. Characterization of the surgical instrument behaviour enables the control of the tip motion. A flexible multibody modelling approach was used to study the sliding behaviour of the instrument inside a curved endoscope. The surgical instrument was modelled as a series of interconnected planar beam elements. The curved endoscope was modelled as a rigid curved tube. A static friction based contact model was implemented. The simulations were carried out both for the insertion of the flexible instrument and for fine manipulation. A computer program SPACAR was used for the modelling and simulation. The simulation result shows the stick-slip behaviour and the motion hysteresis because of the friction. The coefficient of friction has a large influence on the motion hysteresis, whereas the bending rigidity of the instrument has little influence.

J.P. Khatait, D.M. Brouwer, R.G.K.M. Aarts, \& J.L. Herder (2012). Modeling of a flexible instrument to study its sliding behavior inside a curved endoscope, Journal of Computational and Nonlinear Dynamics, 8(3), 031002(Oct 30, 2012)(10 pages). [DOI: 10.1115/1.4007539] 


\subsection{Introduction}

Surgical robotic systems are revolutionizing healthcare and medical services. Minimally-Invasive-Surgery (MIS), also termed as laparoscopic surgery, has greatly reduced the unnecessary damage and trauma to healthy tissues, leading to faster recovery, reduced infection rate, and reduced post-operative complications. Most of the limitations imposed by the conventional laparoscopic system are well addressed by the surgical robotic system by increasing dexterity, restoring proper hand-eye coordination and an ergonomic working position, and improving visualization [14,32]. Furthermore, the ability of integrating and interfacing with various technologies has expanded the horizon of these robotic systems.

The state-of-the-art robotic surgery systems employ rigid instruments [6]. However, with conventional colonoscopy and with the emergence of Natural Orifice Transluminal Endoscopic Surgery (NOTES) and Single Incision Laparoscopic Surgery (SILS) procedures, the use of flexible instruments is inevitable. These flexible instruments are fed through access channels provided in the endoscope or endoscopic platform. The instrument tip is remotely controlled. The inherent flexibility of the instrument, coupled with the friction inside the endoscope channel and the convoluted shape of the endoscope inside the body, makes the control of the instrument tip difficult and cumbersome. As the flexible endoscopy continues evolving more into a therapeutic tool and as the endoscopic procedures are becoming more invasive, the surgical instruments require complex manipulations [7,57]. The instrument tip needs to deliver a certain amount of force or to orient in a particular way. The motion and force fidelity of these instruments is critical for achieving good surgical outcomes.

In an endoscope-like surgical system, the instrument is controlled from the proximal end [57]. Nonlinearities are introduced in motion transmission by the friction forces between the instrument and the access channel. Moreover, the shape of the endoscope is not fixed. It changes depending on the location of the surgical site. There will be a change in the force/torque delivered, which is dependent on the friction properties and the shape of the contacting surfaces. Since it is difficult to place the sensors at the distal end of the instrument, the actual position and the force delivered at the instrument tip are difficult to estimate and control. This makes the control of the instrument tip difficult and challenging.

A thorough understanding of the flexible instrument behaviour inside the access channel of the endoscope can lead to proper design of controller and 
eventually leads to automatic control of the instrument tip for desired motion or force. This also leads to design of the instruments not only for the functionality but also for the control. The focus of this paper is to understand the sliding behaviour of the flexible instrument inside a curved endoscope in the presence of friction. The shape of the curve and the bending rigidity of the instrument can have large influence on the sliding behaviour of the instrument. The rotational behaviour of the instrument and the experimental validation of simulation results will be addressed in future work and, therefore, out of the scope of this paper. This paper addresses only the unloaded case, i.e., there is no load applied at the instrument tip.

A flexible multibody modelling approach has been used in the past to study the nonlinear dynamic behaviour of a flexible drive-shaft. Ten Hoff [60] has used a flexible multibody model to study the behaviour of a catheter for intravascular imaging. Jansen [19] has used similar approach for the modelling of oil drillstring to study its nonlinear dynamics. Though the application is very diverse, the nature of the problem is very similar. In this paper, the flexible multibody modelling approach was also used.

In this paper, an endoscope refers to flexible endoscope typically used for the examination of gastrointestinal tract, for example, during colonoscopy and gastroscopy procedures. The instrument refers to the flexible instruments used for biopsy or for simple surgical procedures, which are fed through the access channel of the endoscope. The proximal end of the instrument is the base end from where the surgeon manipulates the instrument. The distal end is the tip of the instrument which interacts with the tissue directly.

The model of the instrument was defined using similar approach. The modelling of the flexible surgical instrument is described in Section 2.2. The model of the flexible instrument together with that of the curved endoscope is given. Contact model is also described. Section 2.3 describes the simulation for the insertion and fine manipulation of the instrument. Translation motion was given as an input motion to the proximal end. The forces exerted at the distal node and all intermediate nodes were observed in the presence of different coefficients of friction. Simulations were carried out for fine manipulation by giving sinusoidal motion along the axis of the tube at the proximal end. The motion at the end tip were observed for different coefficients of friction and for different values of bending rigidities for the instrument. The effect of friction and that of bending rigidity were studied and discussed in Section 2.3.2. The modelling and simulation of the instrument inside the curved tube is discussed in Section 2.4. The conclusion is presented at the end. 


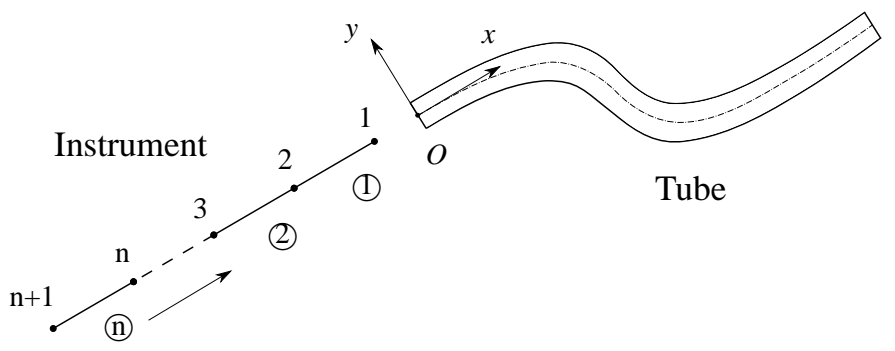

Fig. 2.1: Model of the instrument with the curved tube at the beginning of insertion

\subsection{Modelling of a Surgical Instrument Inside a Curved Endoscope}

A flexible multibody modelling approach was used for the modelling of a flexible surgical instrument inside an access channel of a curved endoscope. The surgical instrument was modelled as a series of interconnected two-noded beam elements. The beam elements were connected in series, defining the full length of the instrument. A curved rigid tube of uniform circular cross-section was used to define the shape of the access channel of the curved endoscope. The shape of the curved tube was defined by a centre line. The size of the access channel was defined by the tube diameter. As the instrument was fed through the access channel and being manipulated, the instrument was always confined within the access channel. The contact between the instrument and the inside wall of the access channel was defined at the nodes of the beam elements. As the node penetrates the inside wall of the tube, reaction force is exerted at the nodes.

A computer program, SPACAR [21], is used for the modelling and simulation of the flexible surgical instrument inside a curved tube. SPACAR is a modelling and simulation tool based on finite element method (FEM) for multibody dynamic analysis of planar and spatial mechanisms and manipulators with flexible links.

The model of the flexible instrument together with the model of the curved tube is shown in Fig. 2.1. The origin of the fixed coordinate system, $\mathrm{O}$, is situated at the beginning of the tube and the initial tangential direction is the $x$-axis. The encircled number, (II, represents the $n^{\text {th }}$ beam element. The node 1 is the tip of the instrument.

The FEM model of the instrument is described in Section 2.2.1. The model of the curved endoscope is presented in Section 2.2.2. Parametric form of a 
Bézier curve is given. The equation for the orthogonal vectors at the point of contact is presented. Contact model is given in Section 2.2.3. Normal force because of interaction at the point of contact is obtained. Depending on the sliding motion at the point of contact, tangential force is exerted at the node based on the friction model.

\subsubsection{Finite Element Model of the Surgical Instrument}

The surgical instrument was modelled as a series of interconnected planar beams elements. The planar beam elements are connected at common nodal points and the assembly of all elements describes the entire length of the instrument. The finite element model of the instrument is shown in Fig. 2.1.

The location of each element $k$ is described relative to a fixed coordinate system $(x y)$ by a set of nodal coordinates $\boldsymbol{x}^{(k)}$. It includes the Cartesian coordinates of the element nodes and the rotation angles describing the angular orientation of the base vectors rigidly attached at the nodes.

With respect to some reference configuration of the element, the instantaneous values of the nodal coordinates represent a fixed number of independent deformation modes for the element. The deformation modes are specified by a set of generalized deformation mode coordinates $e_{i}^{(k)}$. The vector of generalized deformation mode coordinates $\boldsymbol{e}^{(k)}$ is expressed as analytical function of the vector of element nodal coordinates $\boldsymbol{x}^{(k)}$. These functions are known as deformation functions. They are expressed as a vector function $\mathcal{D}^{(k)}$, as follows [21,63]

$$
\boldsymbol{e}^{(k)}=\mathcal{D}^{(k)}\left(\boldsymbol{x}^{(k)}\right)
$$

or, in component form

$$
e_{i}^{(k)}=\mathcal{D}_{i}^{(k)}\left(\boldsymbol{x}^{(k)}\right)
$$

where $i$ represents the deformation mode number. The number of deformation modes is equal to the number of nodal coordinates minus the number of degrees of freedom of the element as a rigid body.

A detailed description of the planar beam element is given in Appendix A. The kinematic and dynamic model is described in the following section with a discussion on the practical limitations of the model.

\section{Equations of Motion}

Kinematic Analysis

The kinematic model was built up by assembling the finite elements. The 
elements are connected at the common nodal points. The vector of nodal coordinates $\boldsymbol{x}$, thus formed, defines the configuration of the instrument. The vector $\boldsymbol{x}$ is, therefore, given by

$$
\boldsymbol{x}=\left[\begin{array}{llll}
x_{1} & x_{2} & \ldots & x_{n x}
\end{array}\right]^{T}
$$

where $n x$ represents the total number of nodal coordinates.

The vector of deformation modes $\boldsymbol{e}$ of all elements are then written as a vector function of the nodal coordinates

$$
\left[\begin{array}{c}
e_{1} \\
\vdots \\
e_{n e}
\end{array}\right]=\left[\begin{array}{c}
\mathcal{D}_{1}(\boldsymbol{x}) \\
\vdots \\
\mathcal{D}_{n e}(\boldsymbol{x})
\end{array}\right]
$$

or,

$$
\boldsymbol{e}=\mathcal{D}(\boldsymbol{x})
$$

where $n e$ is the number of deformation mode coordinates. Kinematic constraints are introduced by imposing conditions on both nodal coordinates $x_{i}$ and deformation mode coordinates $e_{i}$.

The configuration and the deformation state of the instrument is defined by the independent generalized coordinates $q_{i}$. The vector of all the independent generalized coordinates $\boldsymbol{q}$ is, therefore, called the vector of degrees of freedom. It includes the vector of independent generalized nodal coordinates $\boldsymbol{x}^{(m)}$ and the vector of independent deformation mode coordinates $\boldsymbol{e}^{(m)}$

$$
\boldsymbol{q}=\left[\begin{array}{l}
\boldsymbol{x}^{(m)} \\
\boldsymbol{e}^{(m)}
\end{array}\right]
$$

The motion of the instrument is described by the vector $\boldsymbol{q}$. The solution is expressed by the functions as

$$
\begin{aligned}
\boldsymbol{x} & =\mathcal{F}^{(x)}(\boldsymbol{q}) \\
\boldsymbol{e} & =\mathcal{F}^{(e)}(\boldsymbol{q})
\end{aligned}
$$

where $\mathcal{F}^{(x)}$ and $\mathcal{F}^{(e)}$ are called the geometric transfer functions. The geometric transfer functions cannot be calculated explicitly from Eqn. (2.5). They have to be numerically determined in an iterative way [21].

The velocity vectors $\dot{\boldsymbol{x}}$ and $\dot{\boldsymbol{e}}$ are obtained by differentiating Eqn. (2.7) with respect to time

$$
\begin{aligned}
\dot{\boldsymbol{x}} & =\mathbf{D} \mathcal{F}^{(x)} \dot{\boldsymbol{q}} \\
\dot{\boldsymbol{e}} & =\mathbf{D} \mathcal{F}^{(e)} \dot{\boldsymbol{q}}
\end{aligned}
$$


where the differentiation operator $\mathbf{D}$ represents the partial differentiation with respect to the vector $\boldsymbol{q}$. Here, $\mathbf{D} \mathcal{F}^{(x)}$ and $\mathbf{D} \mathcal{F}^{(e)}$ are known as the first-order geometric transfer functions. Differentiating Eqn. (2.8) again with respect to time yields the acceleration vectors $\ddot{\boldsymbol{x}}$ and $\ddot{\boldsymbol{e}}$

$$
\begin{aligned}
\ddot{\boldsymbol{x}} & =\left(\mathbf{D}^{2} \mathcal{F}^{(x)} \dot{\boldsymbol{q}}\right) \dot{\boldsymbol{q}}+\mathbf{D} \mathcal{F}^{(x)} \ddot{\boldsymbol{q}} \\
\ddot{\boldsymbol{e}} & =\left(\mathbf{D}^{2} \mathcal{F}^{(e)} \dot{\boldsymbol{q}}\right) \dot{\boldsymbol{q}}+\mathbf{D} \mathcal{F}^{(e)} \ddot{\boldsymbol{q}}
\end{aligned}
$$

where $\mathbf{D}^{2} \mathcal{F}^{(x)}$ and $\mathbf{D}^{2} \mathcal{F}^{(e)}$ are the second-order geometric transfer functions.

\section{Dynamic Analysis}

In a dynamic analysis, the equation of motion is solved. The equations relate forces and torques to positions, velocities, and accelerations. The inertia properties of the concentrated and distributed mass of the elements are described by means of lumped and consistent mass matrices [20]. The global mass matrix is obtained by assembling the lumped and consistent element mass matrices. Stiffness properties are also defined for the flexible elements by means of stiffness matrices relating stress resultants and deformations.

The equations of motion are derived using the principle of virtual power and the principle of d'Alembert. By making use of the geometric transfer function (Eqn. (2.7)), the equations of motion are written as a set of second order ordinary differential equations for the independent generalized coordinates $\boldsymbol{q}$. The detailed description and derivation of kinematic and dynamic analysis of elements are beyond the scope of this paper and the readers are referred to [20,63].

\section{Numerical Integration Methods}

The equations of motion are solved numerically using SPACAR. It has a comprehensive list of integrators, which includes various explicit, implicit, and semi-implicit methods [41,53]. It has Shampine-Gordon based multistep predictorcorrector method as the default integrator. This is an explicit method with a variable time step. Explicit methods of Runge-Kutta type of different orders, and of fixed and variable step size, are also available. A family of semi-implicit methods, which belong to the class of Runge-Kutta-Rosenbrock methods, can be also used.

We used the Shampine-Gordon based integrator for this problem. An initial time step of $1.0 \times 10^{-3} \mathrm{~s}$ was chosen. An error tolerance of $5.0 \times 10^{-7}$ was used for the solution. The error tolerance is used to control the step size in each step of the integration process. 


\section{Discussion}

To describe the complex shape of the surgical instrument, multiple beam elements are connected. More beam elements are required to accurately model the instrument behaviour inside a tube with a complex shape. The higher the curvature of the tube, the smaller the length of beam element is required. That also means more beam elements are required for the same length of the instrument. A length ratio $\left(l_{e} / R_{\min }\right)$ can be defined, where $l_{e}$ is the element length and $R_{\min }$ is the minimum radius of curvature of the tube. As the instrument is always confined within the tube, this also ensures limited deformation within an individual element. This is also equal to the angle subtended by the element at the centre of curvature. Moreover, more elements also mean high computational time required for the simulation. An element length, corresponding to $15 \mathrm{deg}$ angle subtended, has been used for the study of rotation transmission behaviour of a flexible drive-shaft [60]. This corresponds to the length ratio of 0.26 . This is used as the basis for the selection of number of elements.

In the simulations, the curvature of the tube is reduced with respect to real anatomical features. This leads to fewer number of elements required to capture the typical characteristics without unduly increasing the computation time. This paper focuses on characteristic behaviour of the instrument inside a curved tube in translation. However, modelling is an iterative process and depending on accuracy required, the model may have to be refined.

\subsubsection{Model of an Access Channel of a Curved Endoscope}

The access channel in the curved endoscope was modelled as a rigid curved tube. The curved shape of the tube was defined by a centre line. The centre line of the tube can be defined by a straight line, a circular arc, a Bézier curve, or a combination of these. Figure 2.2 illustrates a part of a curved tube defined by a Bézier curve. The curve defines the centre line of the tube. The first control point $\mathbf{P}_{\mathbf{1}}$ and the last control point $\mathbf{P}_{\mathbf{4}}$ define the end points of the curve. Intermediate control points $\mathbf{P}_{\mathbf{2}}$ and $\mathbf{P}_{\mathbf{3}}$ influence the path of the curve. The first two and the last two control points define lines which are tangent to the beginning and the end of the curve [9]. Any point $\mathbf{P}(u)=\left[\begin{array}{ll}x(u) & y(u)\end{array}\right]$ on a 


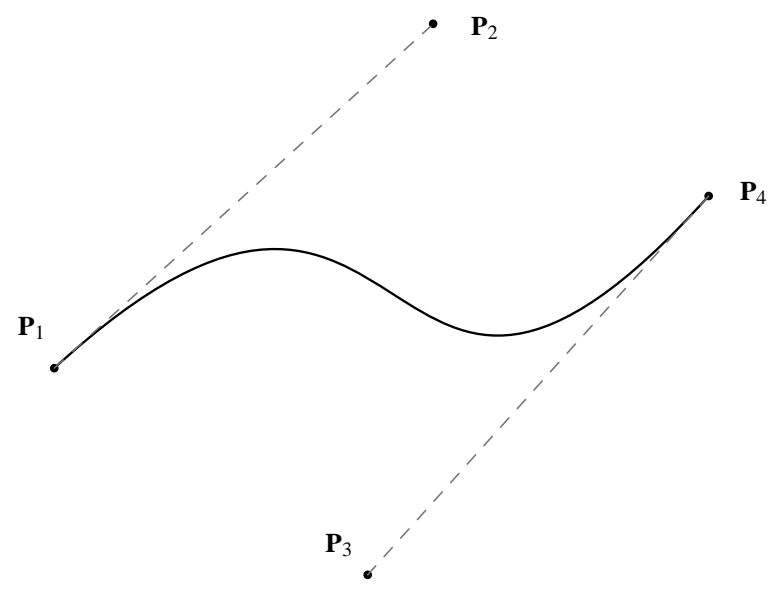

Fig. 2.2: Cubic Bézier curve defined by four points

parametric cubic Bézier curve is given by

$$
\begin{aligned}
\mathbf{P}(u) & =(1-u)^{3} \mathbf{P}_{1}+3 u(1-u)^{2} \mathbf{P}_{2}+3 u^{2}(1-u) \mathbf{P}_{3}+u^{3} \mathbf{P}_{4} \\
& =\left[\begin{array}{llll}
(1-u)^{3} & 3 u(1-u)^{2} & 3 u^{2}(1-u) & u^{3}
\end{array}\right]\left[\begin{array}{l}
\mathbf{P}_{1} \\
\mathbf{P}_{2} \\
\mathbf{P}_{3} \\
\mathbf{P}_{4}
\end{array}\right]
\end{aligned}
$$

where the parameter $0 \leq u \leq 1$ and the control points $\mathbf{P}_{1}, \mathbf{P}_{2}, \mathbf{P}_{3}$, and $\mathbf{P}_{4}$ define a Bézier polygon. Equation (2.10) can be written in algebraic form as

$$
\begin{aligned}
\mathbf{P}(u) & =\mathbf{a}_{1}+\mathbf{a}_{2} u+\mathbf{a}_{3} u^{2}+\mathbf{a}_{4} u^{3} \\
& =\left[\begin{array}{llll}
1 & u & u^{2} & u^{3}
\end{array}\right]\left[\begin{array}{llll}
\mathbf{a}_{1} & \mathbf{a}_{2} & \mathbf{a}_{3} & \mathbf{a}_{4}
\end{array}\right]^{T} \\
& =\left[\begin{array}{llll}
1 & u & u^{2} & u^{3}
\end{array}\right][\mathbf{A}]
\end{aligned}
$$

where $\mathbf{a}_{i}$ is the vector-valued algebraic coefficients. From Eqn. (2.10) and Eqn. (2.11), $\mathbf{A}$ can be written as

$$
\mathbf{A}=\left[\begin{array}{l}
\mathbf{a}_{1} \\
\mathbf{a}_{2} \\
\mathbf{a}_{3} \\
\mathbf{a}_{4}
\end{array}\right]=\left[\begin{array}{l}
\mathbf{P}_{1} \\
-3 \mathbf{P}_{1}+3 \mathbf{P}_{2} \\
3 \mathbf{P}_{1}-6 \mathbf{P}_{2}+3 \mathbf{P}_{3} \\
-\mathbf{P}_{1}+3 \mathbf{P}_{2}-3 \mathbf{P}_{3}+\mathbf{P}_{4}
\end{array}\right]
$$

A tangent vector at any point on the curve is given by the parametric derivative of the curve. The parametric derivative of a cubic Bézier curve from 
Eqn. (2.11) is

$$
\mathbf{P}^{\prime}(u)=\left[\begin{array}{llll}
0 & 1 & 2 u & 3 u^{2}
\end{array}\right][\mathbf{A}]
$$

\section{Normal From a Point $P_{o}$ to the Centre Line}

When the beam is in contact with the inner wall of the tube, the interaction force acts at the node. This requires calculation of the normal and tangent at the contact point. The normal distance between a point $\mathbf{P}_{o}$ and a curve $\mathbf{P}(u)$ is the length of the line perpendicular to a tangent to the curve, as shown in Fig. 2.3. The problem is to find the value of $u$ which locates $\mathbf{P}_{c}$, the point on the curve where a line from $\mathbf{P}_{o}$ is perpendicular to the tangent line. The vector from $\mathbf{P}_{o}$ to the curve is $\left(\mathbf{P}_{c}-\mathbf{P}_{o}\right)$. The tangent vector $\mathbf{P}_{c}^{\prime}$ is given by the parametric derivative of the curve at point $\mathbf{P}_{c}$ (Eqn. (2.13)). For this vector and the tangent vector to be perpendicular

$$
\left(\mathbf{P}_{c}-\mathbf{P}_{o}\right) \cdot \mathbf{P}_{c}^{\prime}=0
$$

In terms of the algebraic coefficients describing $\mathbf{P}_{c}$ (Eqn. (2.11)), Eqn. (2.14) can be expanded to give

$$
c_{0}+c_{1} u+c_{2} u^{2}+c_{3} u^{3}+c_{4} u^{4}+c_{5} u^{5}=0
$$

where

$$
\begin{aligned}
& c_{0}=\mathbf{a}_{1} \cdot \mathbf{a}_{2}-\mathbf{P}_{o} \cdot \mathbf{a}_{2} \\
& c_{1}=2 \mathbf{a}_{1} \cdot \mathbf{a}_{3}+\mathbf{a}_{2} \cdot \mathbf{a}_{2}-2 \mathbf{P}_{o} \cdot \mathbf{a}_{3} \\
& c_{2}=3 \mathbf{a}_{1} \cdot \mathbf{a}_{4}+3 \mathbf{a}_{2} \cdot \mathbf{a}_{3}-3 \mathbf{P}_{o} \cdot \mathbf{a}_{4} \\
& c_{3}=4 \mathbf{a}_{2} \cdot \mathbf{a}_{4}+2 \mathbf{a}_{3} \cdot \mathbf{a}_{3} \\
& c_{4}=5 \mathbf{a}_{3} \cdot \mathbf{a}_{4} \\
& c_{5}=3 \mathbf{a}_{4} \cdot \mathbf{a}_{4}
\end{aligned}
$$

Equation (2.15) is solved for $u$ between 0 and 1, yielding one or more real roots. The point on the Bézier curve, thus obtained by putting the value of $u$ in Eqn. (2.10), locates the point $\mathbf{P}_{c}$. The normal distance $d_{\text {normal }}$, is given by

$$
d_{\text {normal }}=\left|\mathbf{P}_{c}-\mathbf{P}_{o}\right|
$$

If the point $\mathbf{P}_{o}$ is in the vicinity of the curve in such a way that $d_{\text {normal }}$ is smaller than the minimum radius of curvature, $R_{\min }$ of the Bézier curve, there will be a unique solution for the most of the cases, which can represent the anatomical features. Nevertheless, the uniqueness of the solution is checked before the simulation is carried out. 


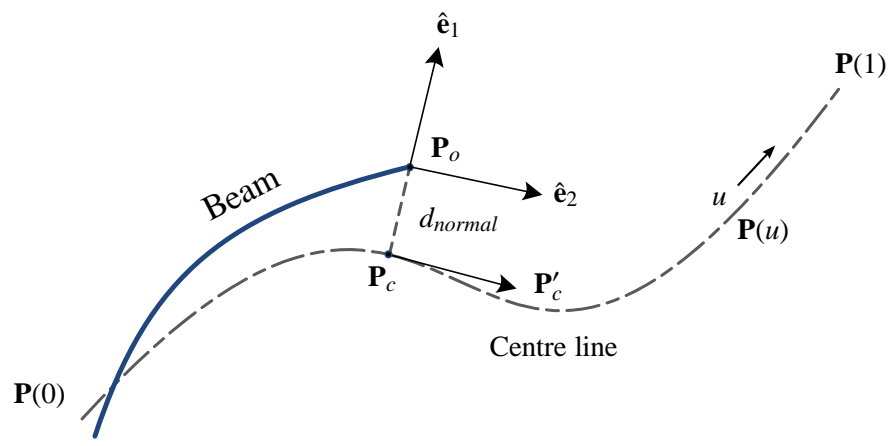

Fig. 2.3: Normal distance between a point, $\mathbf{P}_{o}$, and a Bézier curve. Orthogonal vectors are also shown at the contact node.

\section{Defining Normal and Tangential Directions at the Contact Node}

There are two orthogonal vectors defined at the contact node (Fig. 2.3). The unit vector along the normal $\hat{\mathbf{e}}_{1}$ is defined by

$$
\hat{\mathbf{e}}_{1}=\frac{\left(\mathbf{P}_{o}-\mathbf{P}_{c}\right)}{\left|\mathbf{P}_{o}-\mathbf{P}_{c}\right|}
$$

The tangent at the base point $\mathbf{P}_{c}$ on the centre line of the curve defines the second orthogonal unit vector $\hat{\mathbf{e}}_{2}$

$$
\hat{\mathbf{e}}_{2}=\frac{\mathbf{P}_{c}^{\prime}}{\left|\mathbf{P}_{c}^{\prime}\right|}
$$

\subsubsection{Interaction of Beam with Inner Wall of Tube}

The contact between the beam and the wall is defined at the nodes of the beam elements. As the node approaches the wall, the node experiences an equivalent normal force depending on the depth of penetration and the rate of penetration. Wall stiffness and damping are defined normal to the surface. Friction at the contact point is also defined. Therefore, depending on whether there is any sliding motion at the contact point or not, the node can experience a friction force in the tangential direction.

There are three contact regions defined depending on the position of the node (Fig. 2.4) [60]:

- Region I: No contact

- Region II: Transition 


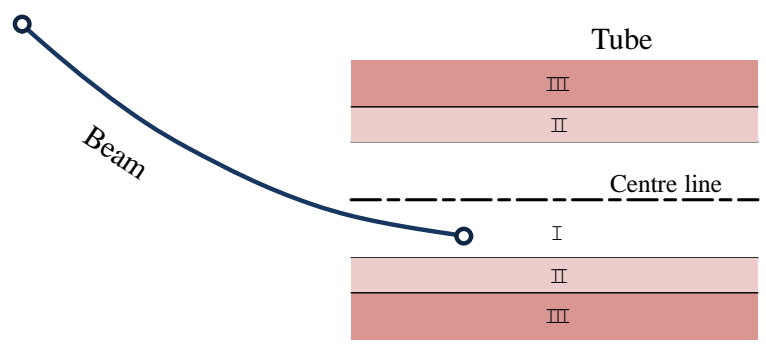

Fig. 2.4: Three regions of contact

- Region III: Full contact

Region I defines the zone when there is no contact at all. Region III is the zone where the beam is in full contact with the inner wall of the tube and there is a linear increase in the normal reaction force with a defined slope (equal to the wall stiffness). Region II defines the transition zone where the reaction force starts increasing from zero as the beam comes in contact with the inner surface of the tube. The $C^{1}$ continuity, that is, the continuity of the force and the tangent stiffness, is considered for the derivation of the fitting polynomial in the transition zone.

Between $a$ and $b$ the reaction force due to the wall stiffness increases according to a second order polynomial (Fig. 2.5). Similarly, the reaction force due to wall damping increases according to a third order polynomial in the transition zone. The damping force is zero in Region I. There is linear damping in Region III.

Therefore, the net normal reaction force $F_{n}$, depending on the normal displacement $x_{n}$ and the normal velocity $v_{n}$, is given by

$$
F_{n}= \begin{cases}0 & \text { if } x_{n}<a \\ -(k / 2)(b-a) \xi^{2}-c_{w}(3-2 \xi) \xi^{2} v_{n} & \text { if } a \leq x_{n} \leq b \\ -k(b-a)(\xi-1 / 2)-c_{w} v_{n} & \text { if } x_{n}>b\end{cases}
$$

where $\xi$ is dimensionless parameter defined as $\xi=\left(x_{n}-a\right) /(b-a), k$ is the wall stiffness, and $c_{w}$ is the wall damping coefficient. Here, $v_{n}$ is the velocity in the normal direction.

\section{Friction Force}

A static friction model, in which the friction force depends on the normal force and the relative velocity only, is used for the calculation of friction forces. The 


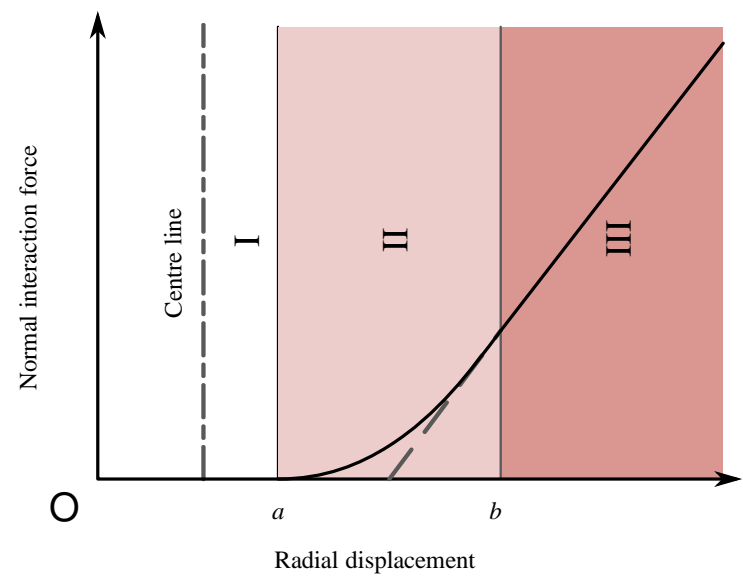

Fig. 2.5: Modelling of contact between the beam and inner wall of the tube

friction model is based on the Coulomb model and has a continuous dependence on the sliding velocity near the zero sliding velocity region

$$
\begin{aligned}
& F_{c}=-\mu F_{n} \\
& F_{t}=F_{c} \tanh \left(c_{v} v_{t}\right)
\end{aligned}
$$

where $\mu$ is the coefficient of friction between the contacting surfaces. Here, $F_{n}$ is the normal reaction force acting at the node (Eqn. (2.19)). Equation (2.20a) gives the Coulomb friction. Equation (2.20b) is the continuous model, where $c_{v}$ is the velocity coefficient which determines the width of the transition region near zero sliding velocity $v_{t}$. Equation $(2.20 \mathrm{~b})$ is used in the simulation as the friction force converges quickly to the Coulomb friction for increasing sliding speeds.

Figure 2.6 shows the comparison of the continuous model for different values of velocity coefficient $c_{v}$. The plots are compared with the Coulomb friction model. The friction force is normalized against the maximum friction force $\mu F_{n}$. The normalized friction force is plotted against the sliding velocity $v_{t}$. The friction force rises continuously and quickly in the transition region near the zero sliding velocity. The transition region is narrower for higher values of the velocity coefficient $c_{v}$. The continuous friction model helps in terms of computation time. However, if the transition region is too narrow, the computation time again starts increasing considerably. Therefore, the parameter $c_{v}$ is optimized in such a way that the model converges quickly and the Coulomb friction is still approached. We used a value of $1.0 \times 10^{4}(\mathrm{~m} / \mathrm{s})^{-1}$ for the velocity coefficient $c_{v}$ in the simulations. 


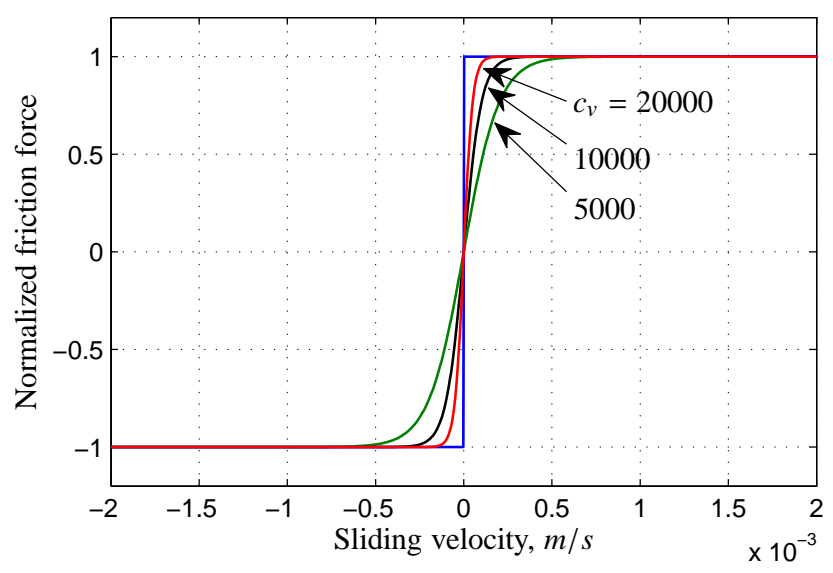

Fig. 2.6: Comparison of continuous friction model based on Coulomb for various values of velocity coefficient, $c_{v}$

\section{Resultant Force Acting at The Node}

A friction force always acts in the opposite direction of the motion on the tangent plane at the point of contact. If $\mathbf{v}$ is the instantaneous velocity vector of the node in contact, it can be written as

$$
\mathbf{v}=\underbrace{\left(\mathbf{v} \cdot \hat{\mathbf{e}}_{1}\right) \hat{\mathbf{e}}_{1}}_{\text {normal to the wall }}+\underbrace{\left(\mathbf{v} \cdot \hat{\mathbf{e}}_{2}\right) \hat{\mathbf{e}}_{2}}_{\text {on tangent plane }}
$$

The first component on the right hand side of Eqn. (2.21) defines the velocity component $\mathbf{v}_{n}$, which is acting normal to the wall. The other component defines the motion on the tangent plane $\mathbf{v}_{t}$. Therefore, the unit vector along the direction of motion on the tangent plane is given by

$$
\hat{\mathbf{e}}_{t}=\frac{\left(\mathbf{v} \cdot \hat{\mathbf{e}}_{2}\right) \hat{\mathbf{e}}_{2}}{\left|\left(\mathbf{v} \cdot \hat{\mathbf{e}}_{2}\right) \hat{\mathbf{e}}_{2}\right|}
$$

Figure 2.7 shows the normal and tangential directions at the point of contact $P$.

Therefore, the resultant force acting at the interacting node by the inner wall of the tube is given by

$$
\mathbf{F}_{t o t}=F_{n} \hat{\mathbf{e}}_{n}+F_{t} \hat{\mathbf{e}}_{t}
$$

where $F_{n}$ and $F_{t}$ are the normal and tangential forces given by Eqn. (2.19) and Eqn. (2.20) respectively. Here, $\hat{\mathbf{e}}_{n}$ is the unit vector along the normal direction and equal to $\hat{\mathbf{e}}_{1}$ (Eqn. (2.17)) and $\hat{\mathbf{e}}_{t}$ the unit vector along the resultant motion in the tangent direction (Eqn. (2.22)). 


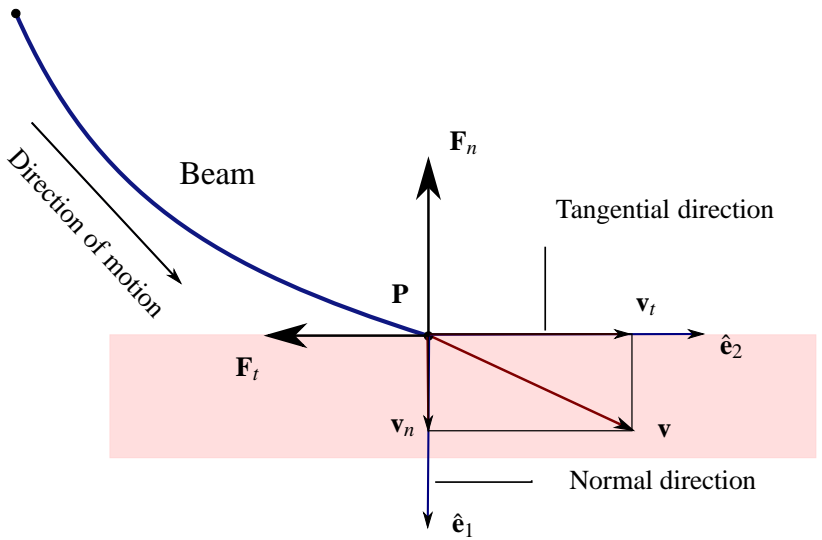

Fig. 2.7: Normal and tangential directions defined at the point of contact

\subsubsection{Discussion}

The model of the instrument is defined using planar beam element. It is assumed that the deformation in the individual elements are small. However, the entire instrument can show large deformation. When the instrument interacts with the wall of the access channel of the instrument, it is assumed that the endoscope does not deform. Though the endoscope itself is very flexible in bending, it may not deform because of the interaction with the instrument in unloaded condition. It is also assumed that there is no interaction with the tissues at the tip. Nevertheless, the magnitude of the resultant force can give an indication whether the assumption is valid. The wall of the tube is defined by wall stiffness and damping ratio. The damping allows better convergence and non-oscillating behaviour.

\subsection{Simulation}

Simulation can provide insight into instrument behaviour during its insertion and fine manipulation thereafter. We are interested in the following attributes of the instrument:

- Force exerted on the instrument by the tube wall

- Maximum stress developed in the instrument

- Motion transmission at the tip

- Force transmission at the tip 
The first two quantities will affect the life time of the instrument. Higher forces exerted on the instrument can lead to higher frictional wear. The maximum stress level provides a guideline to the instrument designers. Motion and force transmission characteristics are important for control purposes. Motion hysteresis can give rise to many control problems. Force transmission is important when the instrument tip is interacting with the tissues.

Table 2.1: Mechanical properties

\begin{tabular}{lllll}
\hline Mechanical Property & Unit & Stainless steel & Kevlar $\left.^{(}\right)$ & Nylon \\
\hline Density, $\rho$ & $\left(10^{3} \mathrm{~kg} / \mathrm{m}^{3}\right)$ & 7.8 & 1.44 & 1.04 \\
Modulus of elasticity, $E$ & $\left(10^{9} \mathrm{~N} / \mathrm{m}^{2}\right)$ & 200 & 80 & 3 \\
\hline
\end{tabular}

Table 2.2: Parameters for FEM calculations

\begin{tabular}{|c|c|c|c|c|}
\hline Parameter & Unit & SS wire & Kevlar $^{\circledR}$ wire & Nylon wire \\
\hline Diameter & $\mathrm{mm}$ & 0.50 & 0.50 & 0.50 \\
\hline Mass/length & $\left(10^{-3} \mathrm{~kg} / \mathrm{m}\right)$ & 1.53 & 0.283 & 0.204 \\
\hline Rotational inertia/length & $\left(10^{-11} \mathrm{~kg} \cdot \mathrm{m}^{2} / \mathrm{m}\right)$ & 2.39 & 0.442 & 0.319 \\
\hline Axial stiffness, $E A$ & $\left(10^{3} \mathrm{~N}\right)$ & 39.3 & 15.7 & 0.589 \\
\hline Bending rigidity, $E I$ & $\left(10^{-6} \mathrm{~N} \cdot \mathrm{m}^{2}\right)$ & 614 & 245 & 9.20 \\
\hline
\end{tabular}

The following parameters are chosen as variables for the simulation:

- Stiffness of the instrument

- Friction between the instrument and the tube

- Shape of the tube

- Clearance in the tube

We chose three different materials—-stainless steel (SS), $\operatorname{Kevlar}^{\circledR}$, and nylon wire-for the study. They can cover different stiffness ranges for the instrument. The material properties are given in Tab. $2.1[10,13]$. The shape of the 
tube can be varied by choosing different shapes for the centreline defining the tube. Circular arcs of $90^{\circ}$ and Bézier curves are used for the simulation. Different friction values $(\mu=0.2,0.5$, and1.0) are used for the various simulations. Clearance in the tube can have a large influence on the instrument behaviour. However, the effect of clearance is not studied in this paper. The clearance can be fixed for the most practical cases and it is minimal. The clearance in all the simulations are fixed and exaggerated to provide more insight.

In SPACAR, the mechanical properties are defined with the following input parameters:

- Mass per unit length

- Axial stiffness, $E A$

- Bending rigidity, $E I$

We chose a wire of diameter $0.5 \mathrm{~mm}$. Input parameters are calculated accordingly for the different materials. They are shown in Tab. 2.2.

\subsubsection{Simulation of Flexible Beam Insertion}

Simulation of the insertion of the flexible instrument is carried out in SPACAR as shown in Fig. 2.1. The instrument is constrained in rotation at the proximal end and the input motion is applied at the node along the axial direction, i.e. the $x$-axis. A constant linear velocity, $0.010 \mathrm{~m} / \mathrm{s}$, is applied at the proximal end till the distal end, the node 1, is already out of the curved tube. A constant acceleration, $0.010 \mathrm{~m} / \mathrm{s}^{2}$, is applied in the beginning. Similarly, a constant deceleration, $0.010 \mathrm{~m} / \mathrm{s}^{2}$, is applied at the end, so that the initial and final velocities are zero (Fig. 2.8). It remains at rest after the insertion. A $0.9 \mathrm{~m}$ length of the instrument is inserted inside the curved tube. After the complete insertion of the instrument, the distal end is already out of the tube. Here, the total length of the instrument considered is $1.0 \mathrm{~m}$. The number of elements used to define the entire length of the instrument is 10 .

The curved tube has circular cross-section. Two inner diameters are defined. $D_{a}$ is the diameter when the transition zone starts, and $D_{b}$ is the diameter when the full contact begins. Here, $D_{a}$ is $4.0 \mathrm{~mm}$ and $D_{b}$ is $5.0 \mathrm{~mm}$. The wall stiffness, $k$, used for the model is $1.0 \times 10^{3} \mathrm{~N} / \mathrm{m}$.

The shape of the tube is defined by an arc of 90 deg with straight sections at both ends of the arc. The radius of the arc is $0.5 \mathrm{~m}$. The arc defines the centre line of the tube. The straight section in the beginning of the curved tube provides a guide way to the instrument and ensures that the instrument 

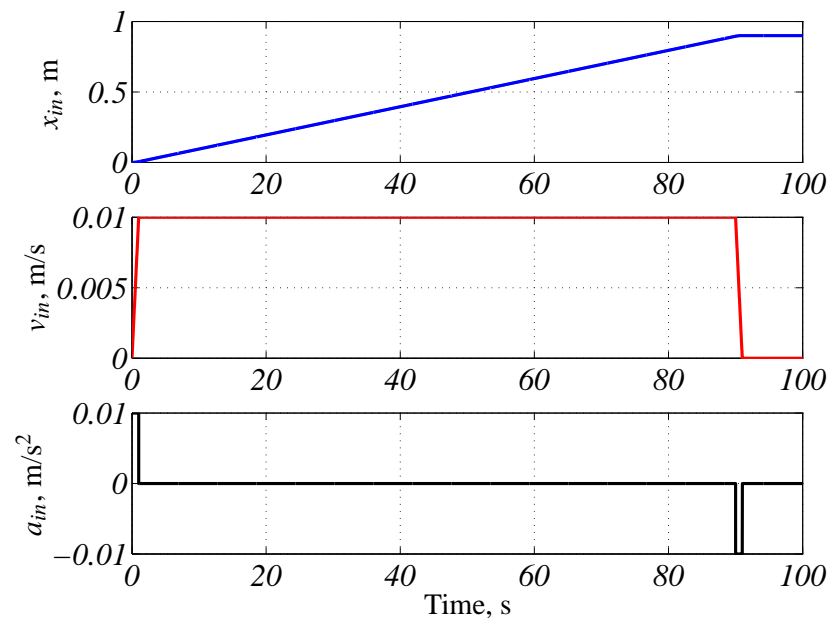

Fig. 2.8: Input motion profile for the insertion

does not buckle under the influence of interacting forces in the beginning of the insertion.

Figure 2.9 shows the plot of forces acting at the first two translational nodes at the distal end when the instrument is inserted into the tube. Only the first two translational nodes are considered for the sake of clarity, though similar plots can be obtained for all the nodes. The friction is assumed to be zero. A stainless steel wire of diameter $0.5 \mathrm{~mm}$ is considered for the instrument. $X 1$ and $X 2$ are the nodal positions of the first two translational nodes. $F 1$ and $F 2$ are the total interaction force acting at the respective nodes. It can be observed that the end node is making contact with the outer wall of the tube all the time, whereas the penultimate node is making contact with the inner wall as expected. Figure 2.10 shows the magnitude of forces acting at the first four distal nodes. The penultimate node experiences a larger force in the beginning when the end node started making contact with the tube. As the instrument advances further into the tube, the first two distal nodes experience larger forces compared to other nodes.

Similarly, a tube can be also defined using a Bézier curve. The control points, which define the Bézier curve, are $\mathbf{P}_{\mathbf{1}}(0.0,0.0), \mathbf{P}_{\mathbf{2}}(0.6,0.0), \mathbf{P}_{\mathbf{3}}(0.0,-0.4)$, and $\mathbf{P}_{4}(0.6,-0.4)$. Figure 2.11 shows the plot of forces acting at the first two distal nodes when the instrument is inserted into the tube. Straight sections are defined at the entry and exit of the tube. The friction is assumed to be zero. A stainless steel wire of diameter $0.5 \mathrm{~mm}$ is considered for the instrument. The end node is experiencing a larger force as it goes through the first bend and 


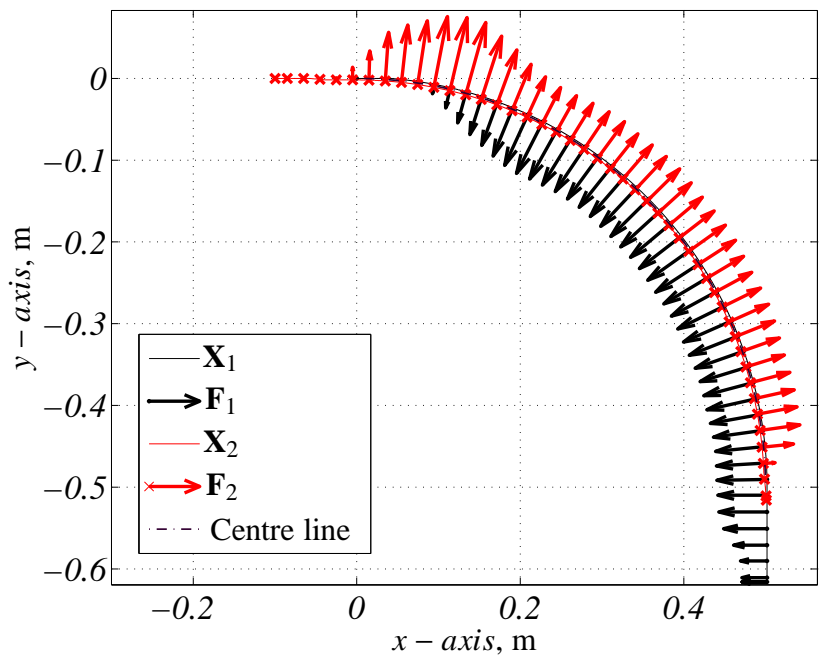

Fig. 2.9: Time history of the forces exerted at nodes 1 and 2 while inserting in circular tube $(\mu=0.0) . F 1$ and $F 2$ are the total interaction force acting at different time instances at the nodes 1 and 2 , respectively.

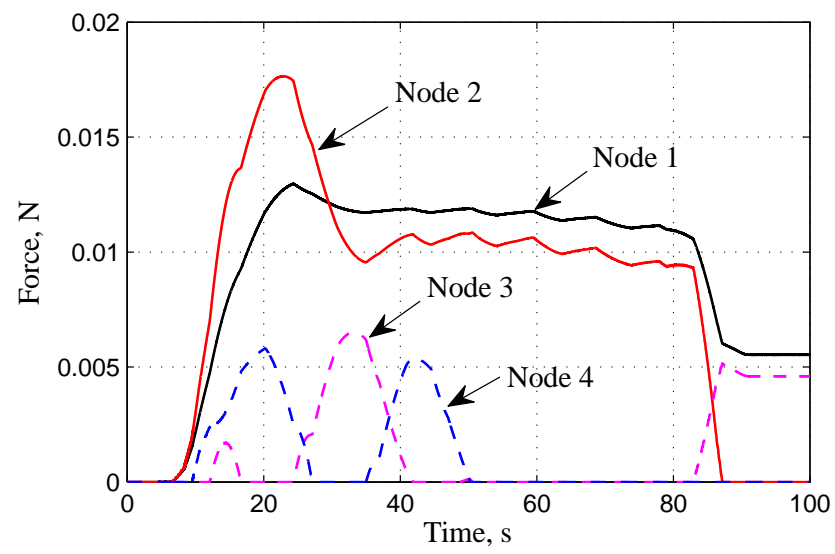

Fig. 2.10: Time history of the forces exerted at first four distal nodes while inserting in circular tube $(\mu=0.0)$

then slowly decreases as the tube straightens. As the node advances further into the second bend, it experiences larger forces again. It can be observed that it is making contact with the concave surface, i.e. the outer wall of the tube. The penultimate node makes contact with the inner wall of the tube and the force acting at the node varies in a similar fashion. Figure 2.12 shows the magnitude of forces acting at the first four distal nodes as it moves inside the 


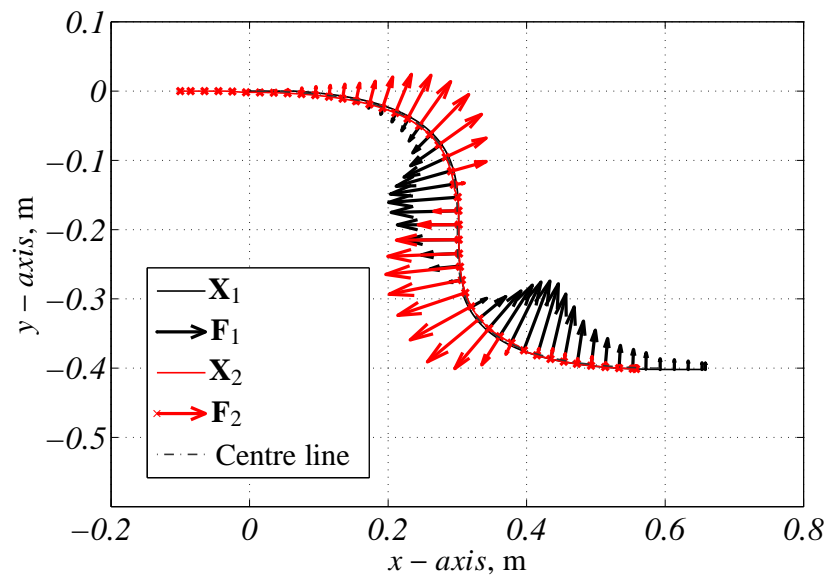

Fig. 2.11: Time history of the forces exerted at nodes 1 and 2 while inserting in a Bézier tube $(\mu=0.0)$. $F 1$ and $F 2$ are the total interaction force acting at different time instances at the nodes 1 and 2 , respectively.

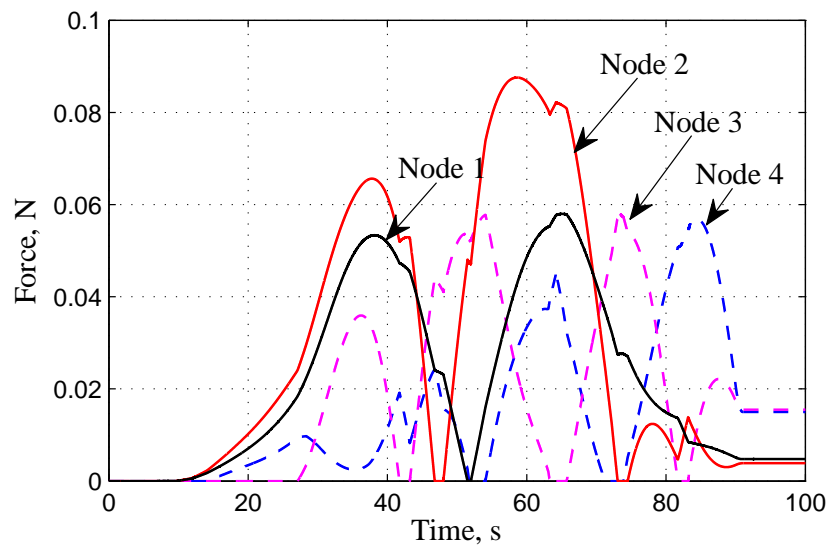

Fig. 2.12: Time history of the forces exerted at first four distal nodes while inserting in a Bézier tube $(\mu=0.0)$

tube. The magnitude of forces acting at the various nodes are higher at the penultimate node. Node 1 is the distal end.

Number of Elements The total number of elements used to define the entire length of the flexible instrument can have significant effect on the characteristic behaviour of the instrument. The total force exerted on the instrument while inserting in the tube can be compared with respect to the total number of elements used. Figure 2.13 shows the plot of the total force exerted by the 


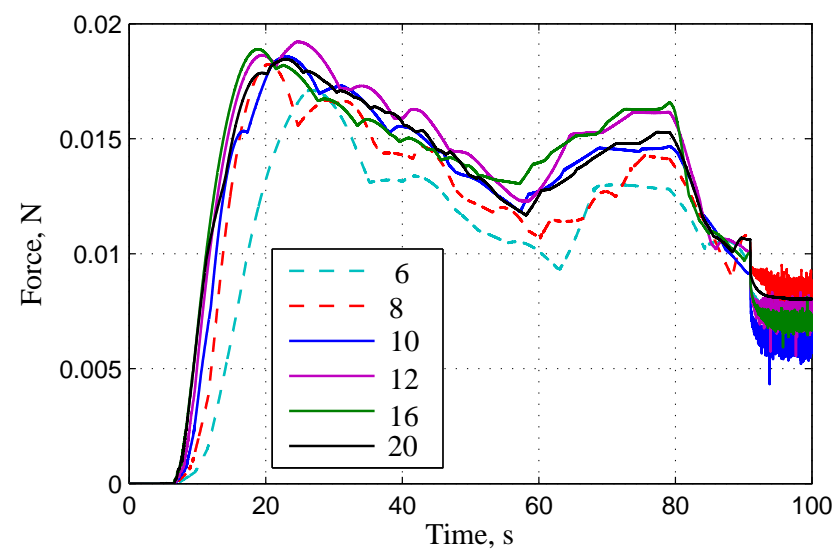

Fig. 2.13: Total force exerted on the instrument while inserting in a circular tube $(\mu=0.5)$. Total force is compared for different number of elements.

wall on the instrument as it advances through the circular tube. The coefficient of friction between the inner wall of the tube and the instrument is 0.5 . The number of elements used are $6,8,10,12,16$, and 20. The figure shows the magnitude of the total force exerted on the instrument.

The plots for 6 and 8 elements show little deviation from the rest. The plots corresponding to $10,12,16$, and 20 elements show convergence. On the other hand, the increased number of elements has a lot of overhead on the computation time. Therefore, we used 10 elements for the flexible instrument for various simulations. However, it is still subjected to the validation and the model can be refined as needed.

\subsubsection{Simulation of Fine Manipulation}

A simulation of fine manipulation is carried out by firstly inserting the instrument completely inside the tube and then manipulating the tip by applying a small stroke input motion to the proximal end. The sine input motion is applied as follows

$$
\begin{aligned}
& x(t)=A \sin (\omega t) \\
& \dot{x}(t)=\omega A \cos (\omega t) \\
& \ddot{x}(t)=-\omega^{2} A \sin (\omega t)
\end{aligned}
$$

where $A$ is the amplitude of motion, and $\omega=2 \pi f_{n}$. Here, the amplitude is chosen as $A=10 \mathrm{~mm}$ and the frequency, $f_{n}$, as $1 \mathrm{~Hz}$. Here, $x(t), \dot{x}(t)$, and $\ddot{x}(t)$ are the displacement, velocity, and acceleration applied at the input node, respec- 


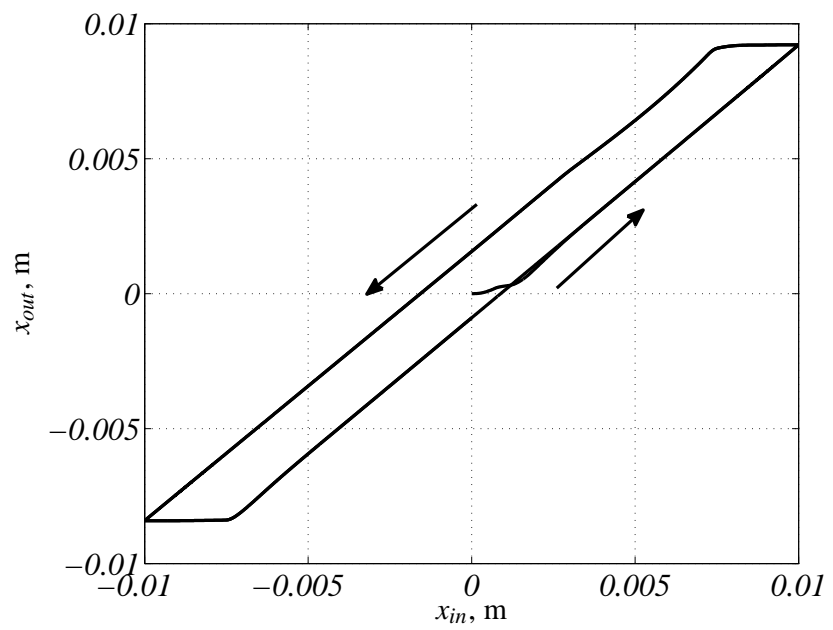

Fig. 2.14: Motion hysteresis in case of circular tube $(\mu=0.2)$

tively. In the present work, we are only considering the unloaded condition. There is no load applied at the instrument tip.

A stainless steel wire of diameter $0.5 \mathrm{~mm}$ is manipulated inside a circular tube. The coefficient of friction, $\mu$, is 0.2 . Figure 2.14 shows the plot of motion hysteresis. Displacement of the end node along the translation axis is plotted against the input displacement. Figure 2.15 shows the comparison of the translation velocity of the output and input node. The instantaneous velocity of the end node along the translation axis is plotted against the input velocity. Stick-slip bahaviour can be observed from the figure. This can lead to sudden movements and it can be very difficult to precisely control the tip motion.

\section{Effect of Friction on Motion Hysteresis}

In order to understand the influence of the friction parameter on motion hysteresis, a stainless steel wire $0.5 \mathrm{~mm}$ in diameter is manipulated inside the Bézier tube for different coefficients of friction $(\mu=0.2,0.5$, and1.0). The displacement of the end node along the translation axis is plotted against the input displacement for each case. Figure 2.16 shows the effect of friction on motion hysteresis. Friction has a large influence on the motion hysteresis. The backlash is larger and even unpredictable as the friction increases. 


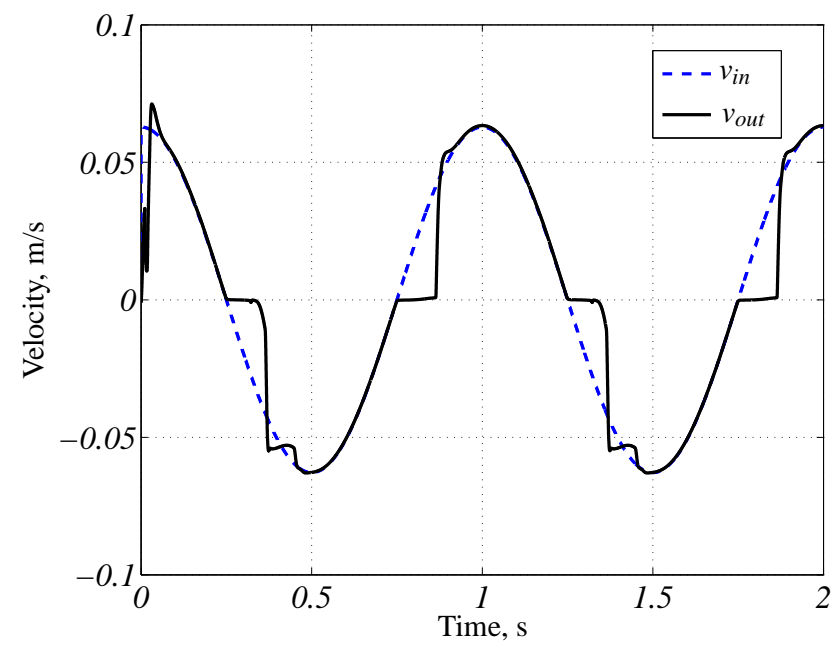

Fig. 2.15: Comparison of translation velocity at input and output $(\mu=0.2)$

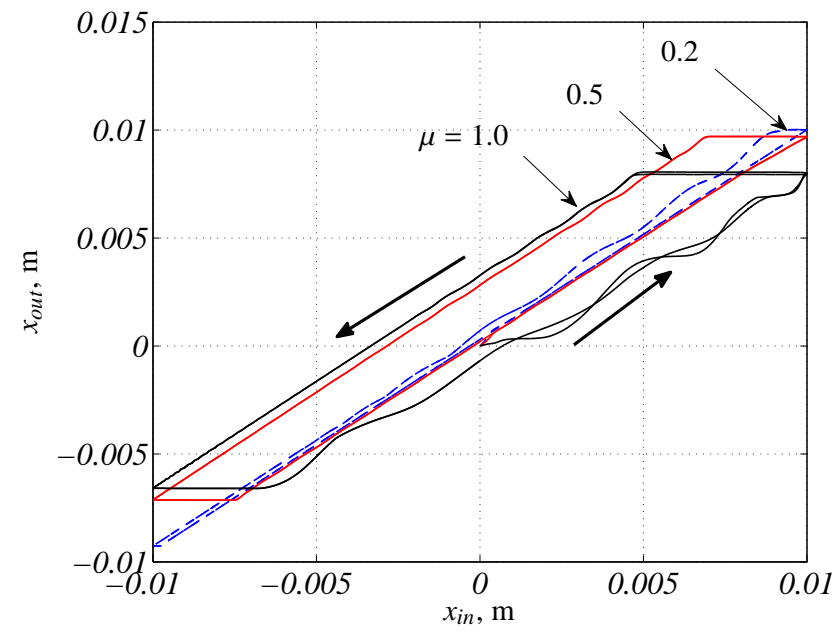

Fig. 2.16: Effect of friction on motion hysteresis in a Bézier tube

\section{Effect of Bending Rigidity on Motion Hysteresis}

The bending rigidity of the instrument can have similar impact on the motion hysteresis. We can get different bending rigidity for the instrument by choosing different material properties. Therefore, we chose stainless steel, $\mathrm{Kevlar}^{\circledR}$, and nylon wires $0.5 \mathrm{~mm}$ in diameter. In this case, we fixed the value of $\mu$ to 0.5 . The same Bézier tube is used as a guiding tube. The dimensions of the tube 


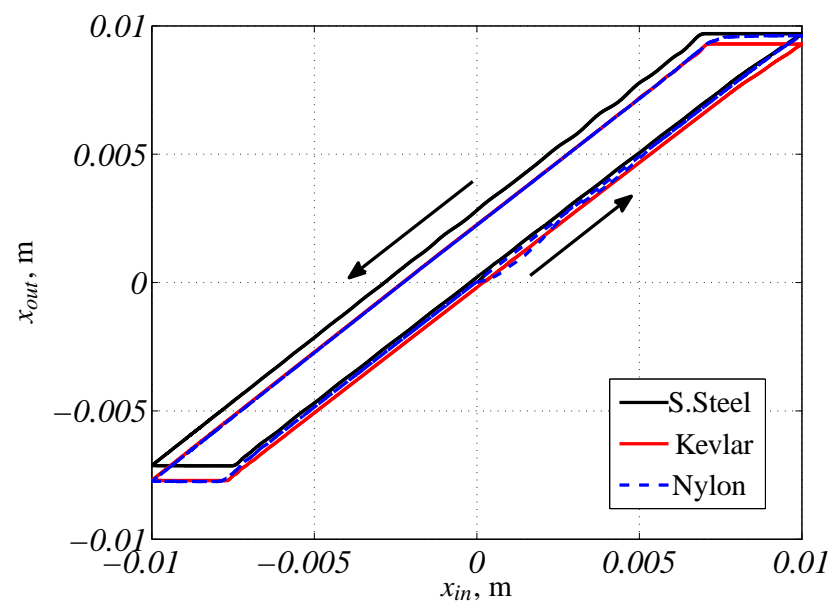

Fig. 2.17: Effect of bending rigidity on motion hysteresis in a Bézier tube $(\mu=0.5)$

and of the instrument are kept constant for all the cases. This allows same clearance between the instrument and the tube. Again, the displacement of the end node is compared with the input displacement for each case. Figure 2.17 shows the influence of the bending rigidity on motion hysteresis. All the three plots show similar variation irrespective of different bending rigidities chosen for the instrument. There is no significant difference observed in the three plots.

\subsection{Discussion}

The simulation of the instrument insertion provides an insight on how the forces get build up because of friction, limited stiffness of the instrument, and the shape of the tube. At the same time it provides initial configuration for the instrument for further small motion hysteresis simulation. The simulation result also shows the stick-slip behaviour because of the friction. This can have very detrimental effect on control of the instrument. The sudden movement of the tip is undesirable and may not be suitable for some surgical procedures. Qualitative study of such phenomenon may not be sufficient and further investigation is necessary.

Motion hysteresis is observed in the simulation results. The coefficient of friction has a large influence. Smaller friction values have very little effect on the motion hysteresis. However, as the friction value is increasing, the motion hysteresis is getting worse (Fig. 2.16). For a higher value of friction, the 
friction force is higher for the same normal reaction force at the contact node. This results into a larger force in the axial direction, and causes compressive load during insertion and tensile force during retraction. This can eventually lead to a large motion hysteresis. In many cases, it may render the instrument useless or it might be even dangerous to use.

The simulation results for the motion hysteresis do not show much dependence on the stiffness values of the instrument for the same value of friction coefficient (Fig. 2.17). This can be expected as the virtual backlash due to friction depends on the normal reaction force and stiffness [55]. In this case both the friction force caused by the normal reaction force, and the driving stiffness of the instrument are directly proportional to the Young's modulus. Therefore the stiffness has no influence on the virtual backlash.

In this paper, only the unloaded case has been considered, i.e. there is no load applied at the instrument tip, and the modelling assumptions may be valid for this case only. The instrument tip interacting with the tissues can have different dynamics and may need a 3-D approach.

The combined translational and rotational behaviour of the instrument requires a 3-D modelling approach and will be presented in the future work. The validation will be addressed separately.

\subsection{Conclusion}

A planar model of a flexible instrument inside a tube was set up. A static friction based contact model was implemented. The motion hysteresis and stick-slip behaviour were observed in the simulations. Friction has a large influence on the performance of the instrument. The motion hysteresis is large for higher value of friction. However, it appears that the bending stiffness has little influence on the motion hysteresis for the same value of friction coefficient.

\section{Acknowledgment}

This research is a part of the TeleFLEX project, which is funded by the Dutch Ministry of Economic Affairs and the Province of Overijssel, within the Pieken in de Delta (PIDON) initiative. We would like to thank M. Krijnen for her contribution in modelling using SPACAR, and J.P. Meijaard for all his support related to modelling and implementation in SPACAR. 



\section{Design of AN EXPERIMENTAL SET-UP to Study the Behavior of a Flexible Surgical Instrument INSIDE AN ENDOSCOPE}

The success of flexible instruments in surgery requires high motion and force fidelity, and controllability of the tip. However, the friction and the limited stiffness of such instruments limit the motion and force transmission of the instrument. In a previous study, we developed a flexible multibody model of a surgical instrument inside an endoscope to study the effect of friction, bending and rotational stiffness of the instrument, and clearance on the motion hysteresis and the force transmission. In this paper, we present the design and evaluation of an experimental set-up for the validation of the flexible multibody model and the characterization of the instruments. A modular design was conceived based on three key functionalities: the actuation from the proximal end, the displacement measurement of the distal end, and the measurement of the interaction force. The exactly constrained actuation module achieves independent translation and rotation of the proximal end. The axial displacement and the rotation of the distal end are measured contactless via a specifically designed air bearing guided cam through laser displacement sensors. The errors in the static measurement are $15 \mu \mathrm{m}$ in translation and 0.15 degree in rotation. Six 1-DOF load cell modules using flexures measure the interaction forces and moments with an error of $0.8 \%$ and $2.5 \%$ respectively. The achieved specifications allow measurement of the characteristic behavior of the instrument inside a curved rigid tube and the validation of the flexible multibody model.

J.P. Khatait, D.M. Brouwer, H.M.J.R. Soemers, R.G.K.M. Aarts, \& J.L. Herder (2013). Design of an experimental set-up to study the behavior of a flexible surgical instrument inside an endoscope, Journal of Medical Devices, 7(3), 031004(Jul 03, 2013)(12 pages). [DOI: 10.1115/1.4024660] 


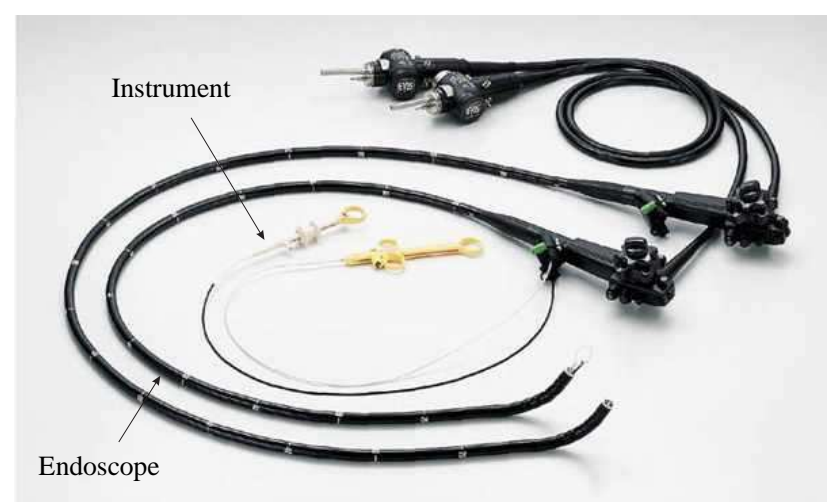

Fig. 3.1: Endoscope with surgical instrument [49]

\subsection{Introduction}

Surgical robotic systems have overcome many of the limitations of conventional laparoscopic systems by increasing dexterity, restoring proper hand-eye coordination and an ergonomic working position, and improving visualization $[6,14,32]$. The surgical robotic systems often employ rigid instruments to perform surgical procedures. However, the use of rigid instruments limits the reach and accessibility to the diseased tissues or organs.

Furthermore, the flexible surgical instruments are increasingly incorporated to perform therapeutic procedures during conventional flexible endoscopy. The emergence of Natural Orifice Transluminal Endoscopic Surgery (NOTES) and Single Incision Laparoscopic Surgery (SILS) procedures necessitates the use of flexible endoscopy in performing more invasive procedures [7,57]. In such interventions, a surgical instrument is fed through an access channel provided in the endoscope or endoscopic platform. Figure 3.1 shows a typical flexible endoscope with a surgical instrument used for colonoscopy [49]. The surgical instrument has limited stiffness so that it can be easily inserted through the endoscope. The motion and force are transmitted to the distal end of the instrument by actuating the proximal end. However, the friction and the limited stiffness of the instrument limit the motion and force fidelity of the instrument. Precise control of these instruments inside the body is critical for achieving successful surgical outcomes.

Modeling and simulation techniques help in developing better insight into the dynamic behavior of a flexible instrument inside an endoscope. This offers an improved design of the instrument and the controller; and eventually leads to improved motion and force fidelity of the instrument. In our previ- 
ous study [26, 28,31], we described the flexible multibody model to study the dynamic behavior of a flexible surgical instrument inside an endoscope. We studied the effect of friction, bending and rotational stiffness of the instrument, and clearance on motion hysteresis. The simulation results showed stick-slip behavior and motion hysteresis. We designed an experimental set-up to validate the model and simulation results.

The design and evaluation of the set-up is discussed in this paper. The design objective is introduced along with the design requirements and the specifications in Section 3.2. The details of the design of the experimental set-up and its evaluation are described in Section 3.3 and Section 3.4 respectively. Section 3.5 discusses the design evaluation. The conclusion is presented in Section 3.6.

\subsection{Design Objective}

The design specifications were chosen with respect to a typical surgical scenariowith a flexible instrument inside an endoscope-where the surgeon controls the instrument from its proximal end. The endoscope provides a rigid support to the instrument. The placement and the orientation of the instrument tip are essential to perform the surgical procedure safely. The instrument tip can carry different surgical tools to perform different procedures. The force delivered at the tip is critical for the successful operation and the safety of the patient as well. During the surgical procedure, the interaction forces will be applied on the endoscope by the instrument and vice-versa. The friction between the instrument and the endoscope limits the motion and force delivery at the tip. At the same time it also results into non-linear dynamic behavior like stick-slip and motion hysteresis.

The design objectives of the set-up are, therefore, as follows:

1. To manipulate the instrument tip from the proximal end

2. To measure the motion of the instrument tip

3. To measure the force of interaction between the instrument and the endoscope

The set-up will be used for validation experiments. As the endoscope is rather stiff as compared to the instrument and is also well supported inside the body cavity by the adjacent organs, the endoscope is modeled as a curved rigid tube. The experiments will also be performed using a curved rigid tube. 
Moreover, the set-up should be able to accommodate different configurations of the rigid tube. It can be further extended to include different kinds of loading at the tip.

\subsubsection{Design Requirements}

The instrument is inserted manually inside the tube until the distal end is out of the tube. The proximal and distal ends are attached to the respective modules for the actuation and measurement respectively. The tube is rigidly fixed on a platform which measures the force of interaction between the instrument and the tube.

The instrument is manipulated both in translation and rotation to perform a surgical procedure. The surgeon gets the visual feedback of the operation. The required resolution [52,54] is of the order of $0.1 \mathrm{~mm}$ in translation and 1 degree in rotation. The range of motion is $\pm 20 \mathrm{~mm}$ in translation and \pm 2 revolutions. The maximum speed can be $20-50 \mathrm{~mm} / \mathrm{s}$ in translation and 5-10 rev/s in rotation. However, the friction induced non-linear dynamic behavior is more prominent at near zero speed. Therefore, the instrument should be able to move at low speed, say, $0.2 \mathrm{~mm} / \mathrm{s}$. Both, rotation and translation, can be varied independently so that we can get a combination of motion.

The force delivery at the tip can be of the order of 5-10 $\mathrm{N}$ with a resolution of $0.01 \mathrm{~N}[47,62]$. The force transmission at the tip is critical for tissue manipulation and for performing surgical procedures like needle steering, suturing, cutting, etc. Essentially, the tip should be able to deliver force and torque in longitudinal direction.

The displacement measurement of the distal end of the instrument can provide vital information about the instrument characteristic behavior. The motion hysteresis and stick-slip behavior can be quantified by measuring and comparing the tip motion with the input motion. The range and resolution of motion at the output are of the same order as of the actuation side. Moreover, during the stick-slip motion, the instrument tip can be released suddenly, and therefore, the required bandwidth for measuring the motion at the tip will be higher than at the proximal end.

When the instrument is manipulated inside the tube, a force is exerted on the instrument by the tube because of friction, the convoluted shape of the tube, and the stiffness of the instrument. The measurement of this interaction force is required in order to study the effect of various parameters on the force of interaction. The range and resolution of the force measurement can be of the order of the force required at the tip for various surgical procedures. 


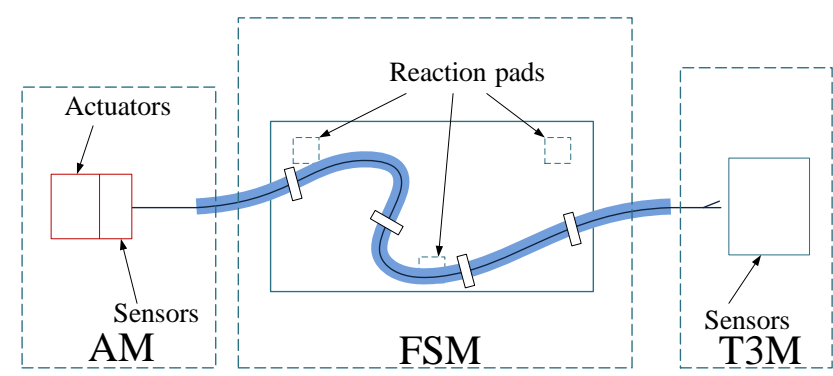

Fig. 3.2: Schematic drawing of the experimental set-up

Apart from these technical requirements, the set-up should be able to cater to other requirements as well. Tubes of different shapes and sizes should be easily mounted. The set-up should be robust and safety features should be included to safeguard the sensors and other critical components. The displacement measurement of the tip of the instrument with contact sensors and components can alter the behavior of the instrument. Therefore, contactless measurement is preferred for the displacement measurement at the tip.

\subsubsection{Design Specifications}

A modular design was sought for the set-up as this would enable easy reconfiguration for future experiments. The experimental set-up, therefore, consists of three basic modules (Fig. 3.2):

1. Actuation module (AM)

2. Force sensing module (FSM)

3. Tip motion measurement module (T3M)

The AM provides necessary actuation along each degree-of-freedom (DOF) required at the proximal end of the instrument, i.e. translation and rotation along the longitudinal axis of the instrument. The AM can be aligned along the entry of the tube and the proximal end of the instrument can be attached to the AM for the actuation.

The FSM enables measurement of forces and torques arising from the interaction of the instrument with the guide tube. A guide tube of different geometric configurations can be rigidly attached on the top plate of the FSM. Load cells can be embedded to measure the forces and torques in all the directions.

The T3M measures the translation and rotation of the distal end of the instrument along the longitudinal axis. The T3M can be aligned along the exit of 
the guide tube and the distal end of the instrument can be attached to the T3M for the displacement measurement.

Based on the design requirements, the design specifications of the three modules-AM, FSM, and T3M-are summarized in Tab. 3.1. The resolution of motion required for the set-up is better than what is required by the surgeon or the surgical procedure as the set-up is intended for the characterization and the model validation of the instrument. The achieved specifications, which will be discussed later in Section 3.4, are also summarized in the table for comparison.

\subsection{Design of the Experimental Set-up}

The conceptual design and the design embodiment of the AM, the FSM, and the T3M are described in detail in the following sections. The experimental set-up also includes the real-time measurement system. The software and hardware are also discussed.

\subsubsection{Design of the AM}

The AM consists of actuation in 2-DOFs - translation and rotation along the longitudinal axis of the instrument. We wanted to have independent actuation for both DOFs, therefore, we chose a stacked configuration. Translation is achieved through a ball screw. A preloaded ball screw nut is used to avoid backlash. However, this adds to additional friction in the drive. The motor encoder is used to measure the translation of the AM.

The rotation axis is mounted on a linear slide guide. The assembly of the ball screw and the slide guide requires alignment of the two axes. If rigidly connected, any misalignment causes increase in the frictional force and deteriorates the repeatability of the set-up. For long stroke motion, the misalignment can even lead to jamming of the stage. Following the principles of exactly constrained design using flexures [55], a coupling is designed to connect the ball screw nut to the slide guide. An exactly constrained design leads to smaller internal forces due to misalignment; and, therefore, it relaxes the alignment requirements — reducing the total effort and cost—during the assembly $[5,54,55]$. 
Table 3.1: Specifications of the set-up

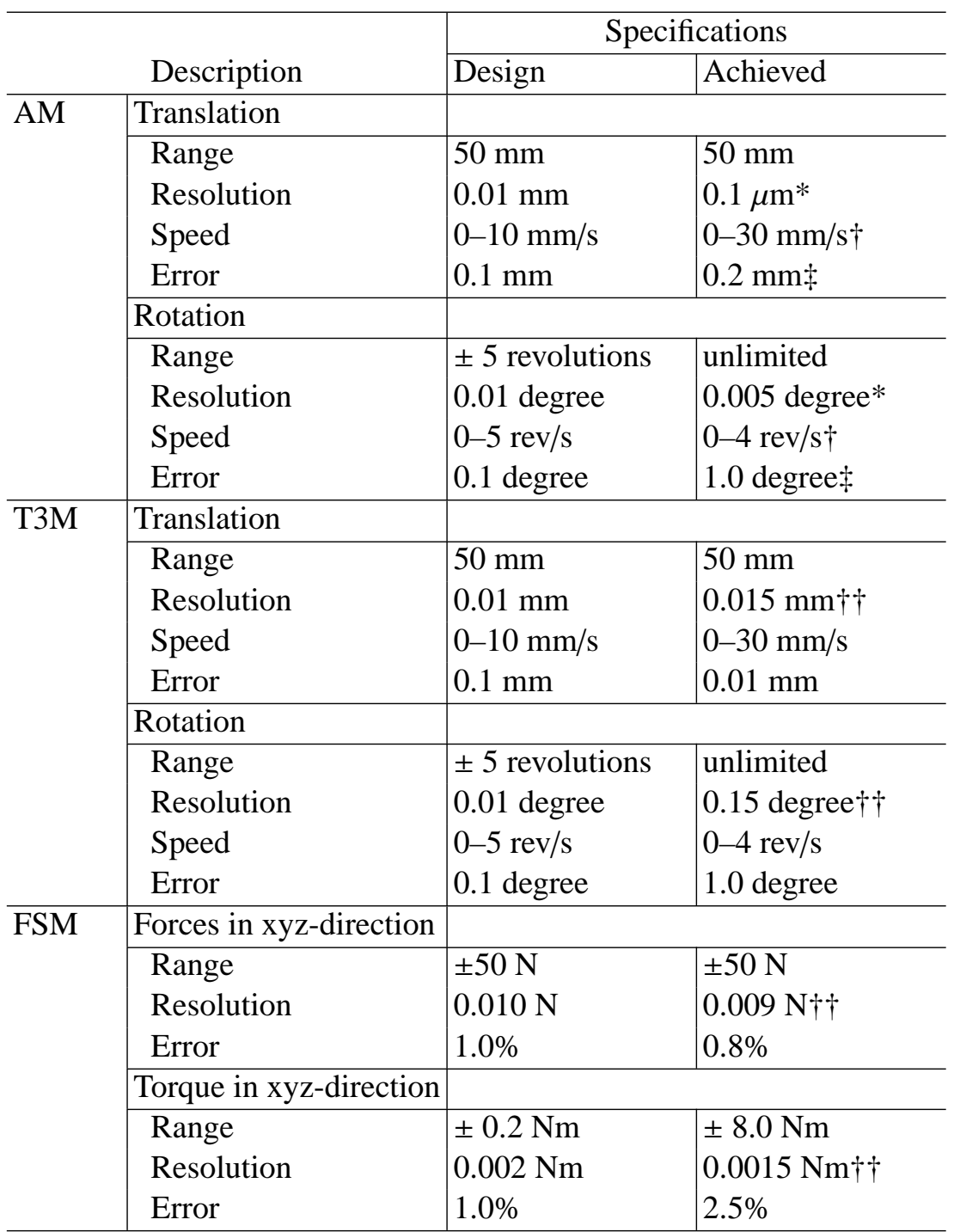

*Based on encoder resolution; $\nmid$ Based on motor speed ; $\ddagger$ Due to gear backlash; $\dagger \dagger$ For cut-off frequency of $45 \mathrm{~Hz}$.

\section{Design of the exactly constrained coupling}

The coupling has to be stiff in the translation and the rotation direction along the translation axis. The ball screw nut should be constrained in rotation so 


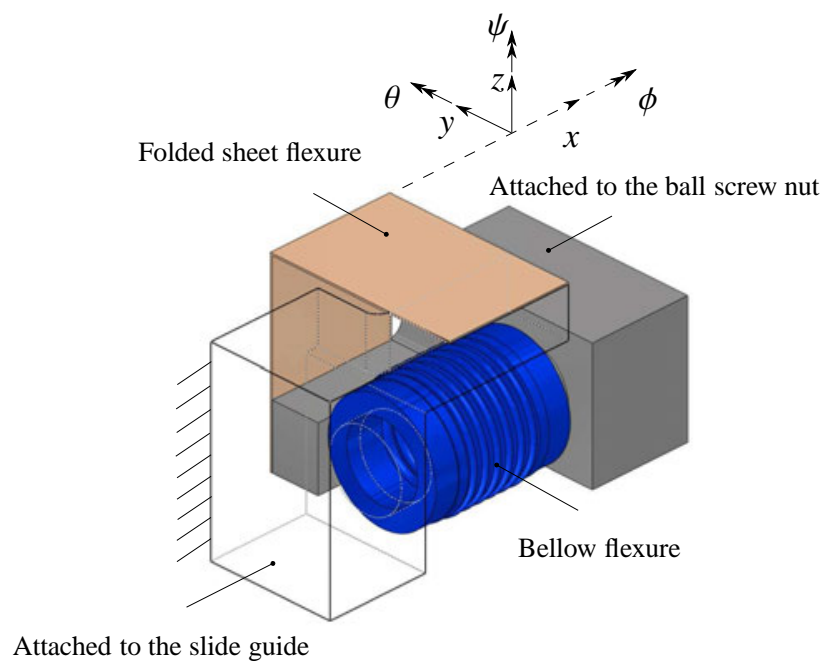

Fig. 3.3: Conceptual design of the coupling for the ball screw and slide guide using a folded sheet flexure and a bellow flexure. The stiff directions are shown with the dashed arrows. The compliant directions are shown with the solid arrows.

that the rotary motion of the ball screw gets converted into the linear motion of the nut. The linear displacement of the nut $x$ is related to the rotation of the ball screw $n$ through the pitch of the ball screw $p$ as $(x=p n)$. A constraint in translation along the same axis transfers the linear motion of the nut to the slide guide. The coupling is compliant in the other 4-DOFs.

An exactly constrained coupling is designed using a bellow flexure and a folded sheet flexure. Figure 3.3 shows the conceptual design of the coupling. A folded sheet flexure $[2,5,55]$ constrains the two ends, parallel to the axis of the fold, only along the axis of fold. All the other 5-DOFs are compliant. Therefore, it can transfer the linear motion from one side to another accurately. Any misalignment can be easily accommodated by the flexure. Likewise, a bellow flexure can constrain the two sides in rotation along the axis of bellow. It is compliant in the other 5-DOFs. The two flexures should be connected in parallel to provide constraints in exactly the 2-DOFs as shown in Fig. 3.3.

The stiffness of the folded sheet flexure along the folding line for equally long sides (with length $l$ ) is given by [55]

$$
k=\left[2\left(\frac{l^{3}}{3 E I}+\frac{6}{5} \frac{l}{G A}\right)\right]^{-1}
$$

where $A$ is the cross-section area, $I$ is the area moment of inertia, $E$ is Young's modulus of elasticity, and $G$ is the Shear modulus of the folded sheet flexure. 
Table 3.2: Specifications of the flexure coupling

\begin{tabular}{l|l}
\hline \multicolumn{1}{c|}{ Description } & Specifications \\
\hline Folded sheet flexure & \\
\hline Stiffness & $1.66 \times 10^{6} \mathrm{~N} / \mathrm{m}$ \\
Allowable offset & $2 \mathrm{~mm}$ \\
\hline Bellow flexure & \\
\hline Torsional stiffness & $220 \mathrm{Nm} / \mathrm{rad}$ \\
Allowable offset & $0.15 \mathrm{~mm}$ \\
Allowable inclination & 2 degree \\
\hline
\end{tabular}

The stiffness value should be such that the friction force in the translation direction should not affect the positional accuracy significantly. Table 3.2 shows the specifications of the folded sheet flexure. Similarly, the bellow flexure is selected for high rotational stiffness. The rotational stiffness of the bellow flexure should be able to provide the reaction torque arising due to the friction between the ball screw and the nut without affecting the accuracy significantly. The specifications are shown in Tab. 3.2.

The flexure coupling should tolerate misalignment because of the inclination and offset distance between the ball screw and the slide guide. The maximum misalignment can be tolerated by the flexure coupling as the DOFs in those directions are released and the coupling is compliant in those directions. The allowable offset and inclination for the bellow flexure looks rather small as it is designed for a longer fatigue life [45]. This is very conservative value for the intended application as it is for non-fatigue use. An offset of about 10 times the specified value can be easily achieved.

\section{Design embodiment of the AM}

A precision ball screw and slide guide were selected [45]. We chose two DC motors for actuating both DOFs [38]. The folded sheet flexure was made out of stainless steel sheet of thickness $0.3 \mathrm{~mm}$, width $20 \mathrm{~mm}$ and length $30 \mathrm{~mm}$ for each side. The bellow flexure was selected and purchased off-the-shelf [45]. Figure 3.4 shows the conceptual design of the AM. The AM included a gear box for the actuation in each DOF. The gear box helped in achieving the lower speed. It also reduced the motor torque required to move the AM initially. 


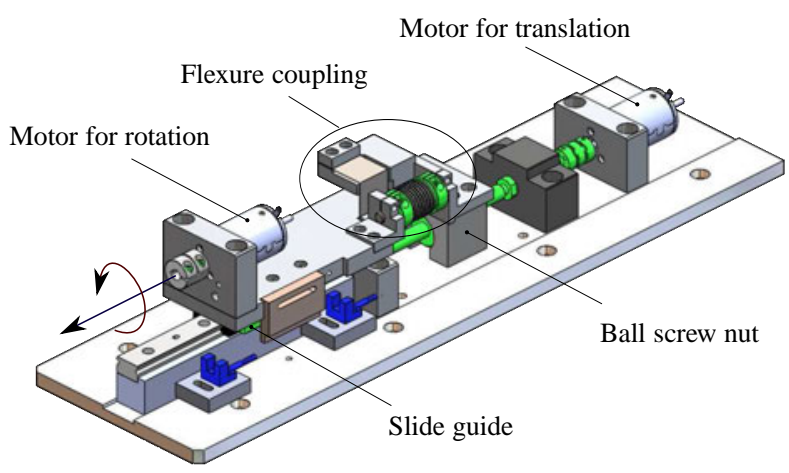

Fig. 3.4: Conceptual design of the AM. The translation and rotation axis is connected via a flexure coupling. The two actuated DOFs are shown by the arrows.

\subsubsection{Design of the FSM}

The FSM was designed to measure forces arising from the interaction between the instrument and the guide tube. The force measurement along six DOFs are required to measure all the possible components of the resultant force. A combination of 1-DOF load cells and $n$-DOFs load cells can be used in various configuration to get a total 6-DOFs force measurement. We chose six 1 -DOF load cells and used the exactly constrained design principles [5,54,55] to design the FSM.

The size of the FSM was chosen so that a curved tube, of the order of human anatomical features, can be accommodated and can be configured easily. Moreover, for the model validation purpose, the curvature of the tube can be relaxed and, therefore, a curved tube of larger dimensions should be also accommodated and can be attached.

\section{Exactly constrained configuration of the FSM}

The conceptual design of an exactly constrained configuration of the FSM using six wire flexures is shown in Fig. 3.5. A wire flexure constrains only one DOF, i.e. along the longitudinal direction. It is compliant in the other five DOFs. Three wire flexures were placed underneath the floating platform along $z$-axis, constraining three DOFs-translation along $z$-axis, and two rotations along $x$ - and $y$-axis. Two wire flexures were placed along $x$-axis, constraining the other two DOFs - translation along $x$-axis and rotation along $z$-axis. One wire flexure was placed along $y$-axis, constraining the translation along $y$-axis. Consequently, the floating top platform was fully constrained by the 

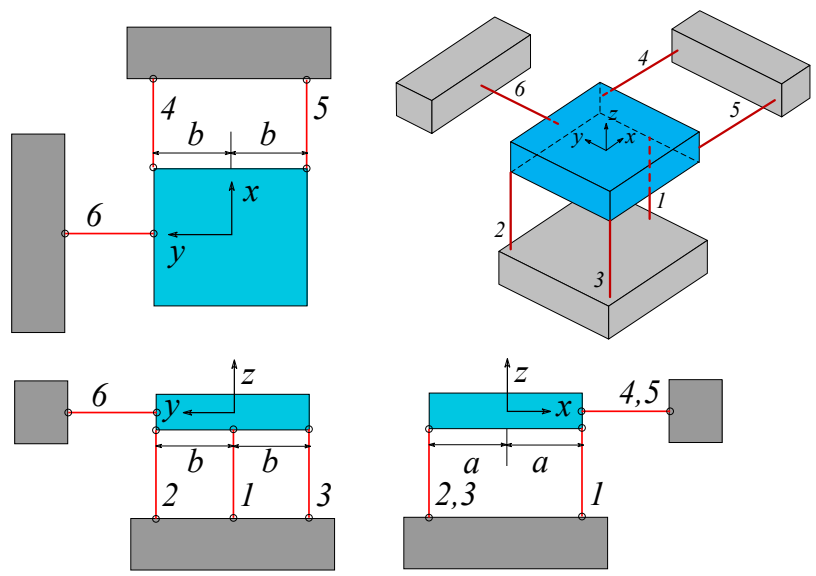

Fig. 3.5: Conceptual design of an exactly constrained configuration of the FSM using six wire flexures

six wire flexures.

The force vector $\mathbf{F}_{x}$, acting at the center of the top plate of the FSM, can be transformed into another force vector $\mathbf{F}_{n}$ as

$$
\mathbf{F}_{x}=A \mathbf{F}_{n}
$$

or,

$$
\left[\begin{array}{c}
F_{x} \\
F_{y} \\
F_{z} \\
M_{x} \\
M_{y} \\
M_{z}
\end{array}\right]=\left[\begin{array}{rrrrrr}
0 & 0 & 0 & -1 & -1 & 0 \\
0 & 0 & 0 & 0 & 0 & -1 \\
1 & 1 & 1 & 0 & 0 & 0 \\
0 & b & -b & 0 & 0 & 0 \\
-a & a & a & 0 & 0 & 0 \\
0 & 0 & 0 & b & -b & 0
\end{array}\right]\left[\begin{array}{c}
F_{1} \\
F_{2} \\
F_{3} \\
F_{4} \\
F_{5} \\
F_{6}
\end{array}\right]
$$

where $\mathbf{F}_{x}$ includes the three force components and three torque components acting at the origin $O$ along $x y z$-directions. $\mathbf{F}_{n}$ includes the forces acting along the six wire flexures. $F_{n}$ is the force component acting along the $n^{\text {th }}$ wire flexure. The dimensions, $a$ and $b$, are defined in Fig. 3.5.

If the stiffness along the compliant direction (also called parasitic stiffness) is negligible as compared to the stiffness in the stiff direction, the force component $F_{n}$ will act only on the load cell attached to the $n^{\text {th }}$ wire flexure.

\section{Design of a 1-DOF load cell module}

Six 1-DOF load cell modules were designed using a wire flexure and a 1-DOF load cell for each module. One end of the wire flexure is attached to the top 


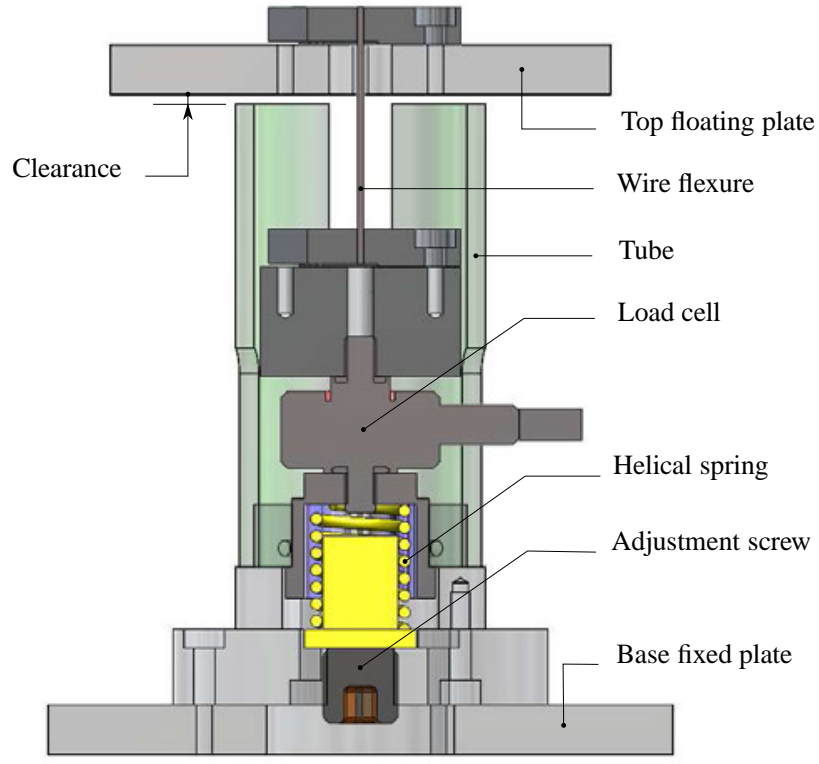

Fig. 3.6: Conceptual design of a 1-DOF load cell module consisting of a wire flexure, a 1-DOF load cell, and a preloading mechanism for overload protection.

plate of the FSM and the other end is attached with the load cell. The load cell is further attached to the base plate through an attachment for overload protection. Figure 3.6 shows the conceptual design of the module. Any force acting along the longitudinal axis of the wire flexure-tension or compression-can be measured by the attached load cell.

A safeguard was designed in order to prevent overload and buckling failure of the wire flexure. The excessive compressive load can lead to the buckling of the wire flexure. An excess load beyond the load cell measurement range can also damage the load cell. Therefore, a safety feature was implemented to limit the magnitude of compressive load. The attachment collapses when the load exceeds a predetermined value. A helical spring was used to preload the attachment. The preloading can be further adjusted by an adjustment screw. If the magnitude of the compressive load acting on the wire flexure is smaller than the preloading, the reaction force is provided by the preloading. However, if the magnitude of the compressive load increases the preloading, the reaction force is provided by the spring; and the spring collapses under the load. There was a small clearance provided between the top plate and the tube. The top plate comes in contact with the tube if the attachment collapses under excessive compressive load and protects the set-up from further damage. 


\section{Dimensioning of the wire flexure}

The wire flexure can be loaded axially both in tension and compression. The compressive load on the wire flexure can lead to buckling and limits the load capacity of the load cell. Therefore, the flexure should be able to support the maximum compressive load which can be measured by the attached load cell without buckling. The critical load $F_{C r}$ for an undeflected wire flexure is given by

$$
F_{C r}=\frac{4 \pi^{2} E I}{l^{2}}=\frac{\pi^{3}}{16} \frac{E d^{4}}{l^{2}}
$$

where $d$ and $l$ are the diameter and the length of the wire flexure respectively.

On the other hand, the wire flexure should be able to accommodate the error because of the misalignment during assembly and the manufacturing tolerances of the components. The maximum allowed deflection in the lateral direction should also include the maximum deformation possible when the other load cell module collapses because of the overload. This prevents the wire flexures from getting damaged even if the FSM is overloaded and collapsed under the load. If $h$ is the maximum allowable deflection in lateral direction, the maximum stress due to bending will be

$$
\sigma_{\max }=\frac{3 E d h}{l^{2}} .
$$

The longitudinal stiffness $k_{a}$ and the lateral stiffness $k_{b}$ of the wire flexure are given by

$$
\begin{aligned}
& k_{a}=\frac{E A}{l}=\frac{\pi}{4} \frac{E d^{2}}{l}, \\
& k_{b}=\frac{12 E I}{l^{3}}=\frac{3 \pi}{16} \frac{E d^{4}}{l^{3}} .
\end{aligned}
$$

From Eqn. (3.5) and Eqn. (3.6), we find that the stiffness ratio $k_{a} / k_{b} \propto(l / d)^{2}$. The wire flexure was designed for the maximum deflection of $1.5 \mathrm{~mm}$ in compliant direction (i.e. in bending) and the maximum compressive load of $100 \mathrm{~N}$ without buckling. A value greater than 1000 was chosen for the stiffness ratio $k_{a} / k_{b}$. A higher value for the stiffness ratio ensures reduced parasitic error. Stainless steel wire of diameter $1.0 \mathrm{~mm}$ and length $30 \mathrm{~mm}$ was chosen for the wire flexure. 


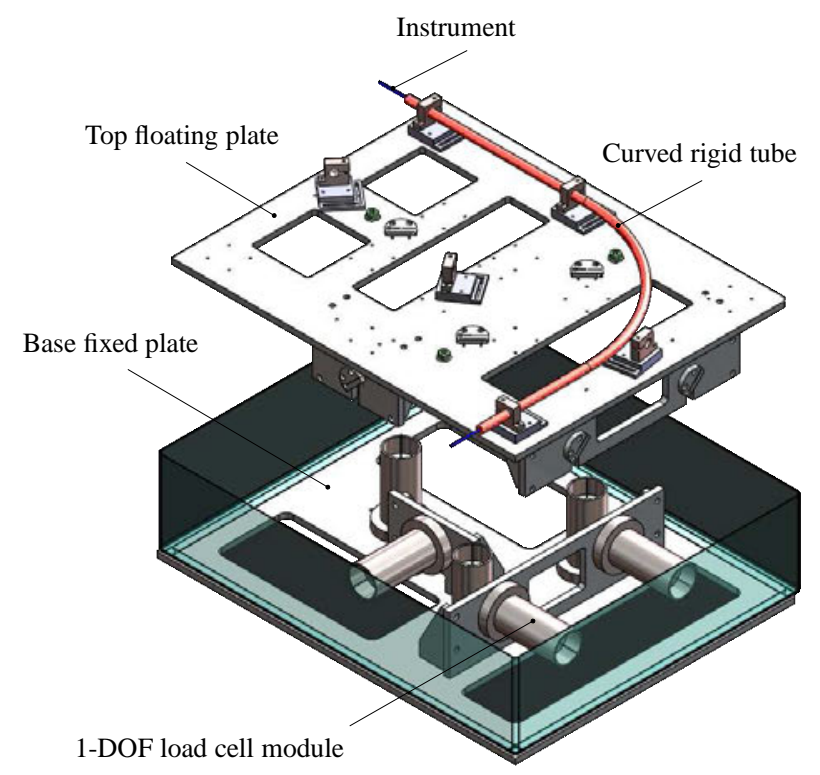

Fig. 3.7: Conceptual design of the FSM with six 1-DOF load cell modules. The six load cell modules are attached to the base fixed plate. The top floating plate will be attached to the ends of six wire flexures.

\section{Design embodiment of the FSM}

Six precision miniature load cells were selected based on the measurement range and the resolution. The load cell measures forces up to $100 \mathrm{~N}$ in both tension and compression with an accuracy of $0.15 \%$ full scale, and with infinite resolution [17]. Firstly, the six 1-DOF load cell modules were assembled as shown in Fig. 3.6. All the load cell modules were mounted on the base plate. The top plate was aligned through three fixtures, and finally clamped to the six wire flexures. Figure 3.7 shows the design embodiment of the FSM.

\subsubsection{Design of the T3M}

The T3M was designed to measure the motion of the tip both in rotation and translation along the longitudinal axis of the instrument at the exit of the curved rigid tube. A contact with the displacement measurement modules can affect the behavior of the instrument. As we want to study the effect of friction between the instrument and the tube, it may be very difficult to account for the output behavior. Therefore, contactless measurement is preferred. Moreover, since we are interested in displacement measurements in only two DOFs, i.e. the rotation and translation along the instrument axis, the displacements in 
other four DOFs are constrained by an air bearing.

Laser displacement sensor (LDS) was chosen for the displacement measurement. The LDS uses optical triangulation as measuring principle. A laser diode projects a visible spot of light onto the target surface. The image of the spot is captured on a high resolution CCD- or CMOS-element. The position of the target is measured by the change in the position of the reflected light on the receiver [44]. Two LDSs were used for measurement in both DOFs. A cam was designed to facilitate measurement in both DOFs simultaneously.

\section{Design of a cam}

A cam is designed with two lobes as shown in Fig. 3.8. The radial displacement $r$ of a point on the cam is linearly dependent on the angular displacement $\theta$ as

$$
r=r_{0}+\left(\frac{r_{1}-r_{0}}{\pi}\right) \bmod (\theta, \pi)
$$

where $r_{0}$ and $r_{1}$ are the radius of the base circle and outer circle enclosing the cam; and $\bmod (\theta, \pi)$ gives the modulus after dividing $\theta$ with $\pi$. Two identical lobes of 180 degree were designed. Therefore, after every half rotation of the cam, the plot of the radial displacement versus the angular displacement repeats, as shown in the figure. As the distance between the cam axis and the LDS, $c_{0}$, is fixed, the displacement $s$ measured by the LDS is directly proportional to the angular displacement of the cam, and is given by

$$
s=\left(c_{0}-r_{0}\right)-\left(\frac{r_{1}-r_{0}}{\pi}\right) \bmod (\theta, \pi) .
$$

The CAD drawing of the designed cam is shown in Fig. 3.9. The plane surface is used to measure the displacement due to translation. The curved surface is used to measure the displacement due to rotation. An undercut is provided to ensure that the laser spot always falls on the curved surface when the cam is rotating.

\section{Design embodiment of the T3M}

The conceptual design of the T3M is shown in Fig. 3.10. The measurement range of the LDS for translation measurement is $50 \mathrm{~mm}$ with a resolution of $25 \mu \mathrm{m}$ at $1.5 \mathrm{kHz}$ [44]. The measurement range of LDS for rotation measurement is $20 \mathrm{~mm}$ with a resolution of $10 \mu \mathrm{m}$ at $1.5 \mathrm{kHz}$. The cam was attached to the tip of the instrument through a guide shaft which was supported on an air bearing. The designed cam profile was obtained through wire EDM. 

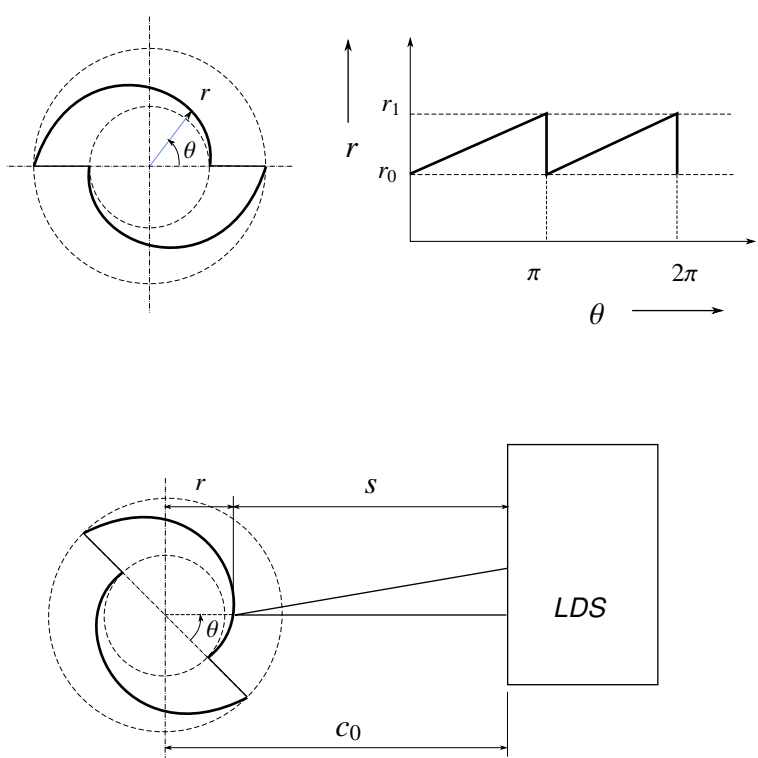

Fig. 3.8: Conceptual design of the cam. The angular displacement of the cam can be measured from the displacement measurement of the LDS.
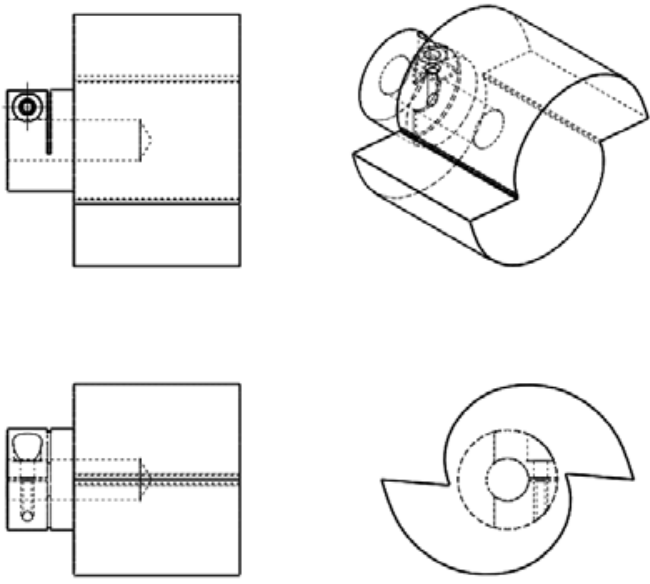

Fig. 3.9: CAD drawing of the cam

\subsubsection{Real-time Measurement System}

The experimental set-up also includes the hardware and software required for real-time measurements and data logging. The measurement system was used 


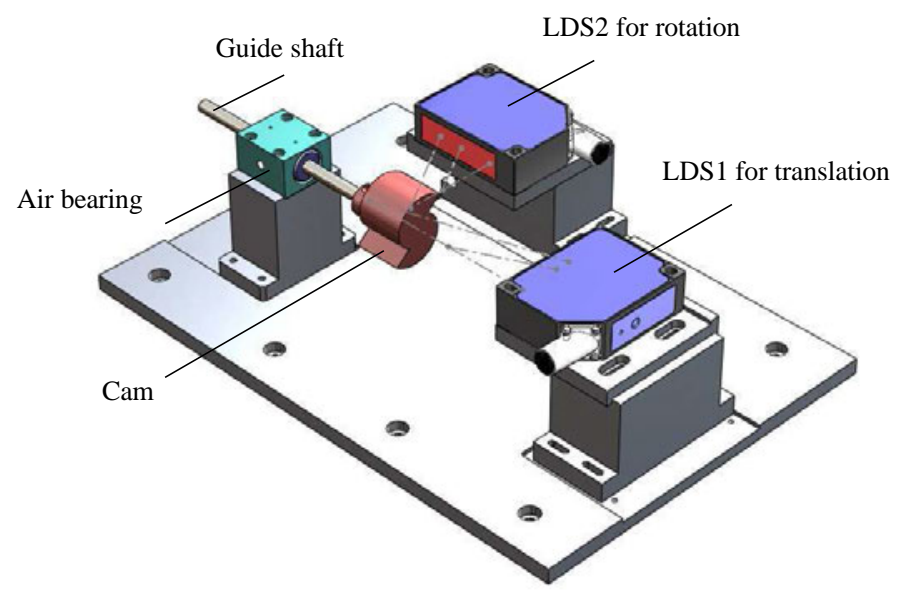

Fig. 3.10: Conceptual design of the T3M

to evaluate the set-up, and will be used later to characterize the behavior of the flexible instrument and to control the instrument tip motion and force.

An xPC target computer was used together with a host computer running Matlab [36]. An xPC target application was created from a Simulink model and downloaded to the target computer from the host computer via a LAN (ethernet) connection.

A multi-function I/O card was selected for data acquisition (DAQ) and control application. The DAQ card had an 8-channel fast 14-bit A/D converter with simultaneous sample/hold circuit, 8 independent 14-bit D/A converters, 8-bit digital input port and 8-bit digital output port, 4 quadrature encoder inputs with single-ended or differential interface and 5 timers/counters [18].

The outputs from the six load-cells were first connected to the amplifiers and then the amplified outputs were connected to the six A/D channels. The other two A/D channels were connected to the two LDS. The two D/A channels were connected to the two DC motor controllers. The two encoders of the DC motors were connected to the two quadrature encoder inputs. Two optical switches were used to limit the stroke of the AM translation, which were connected to the digital input port. One of the switches was used for the homing of the AM translation.

\subsubsection{Discussion}

Figure 3.11 shows the assembly of the experimental set-up, indicating the three modules together with the key components. The curved rigid tube was fixed 


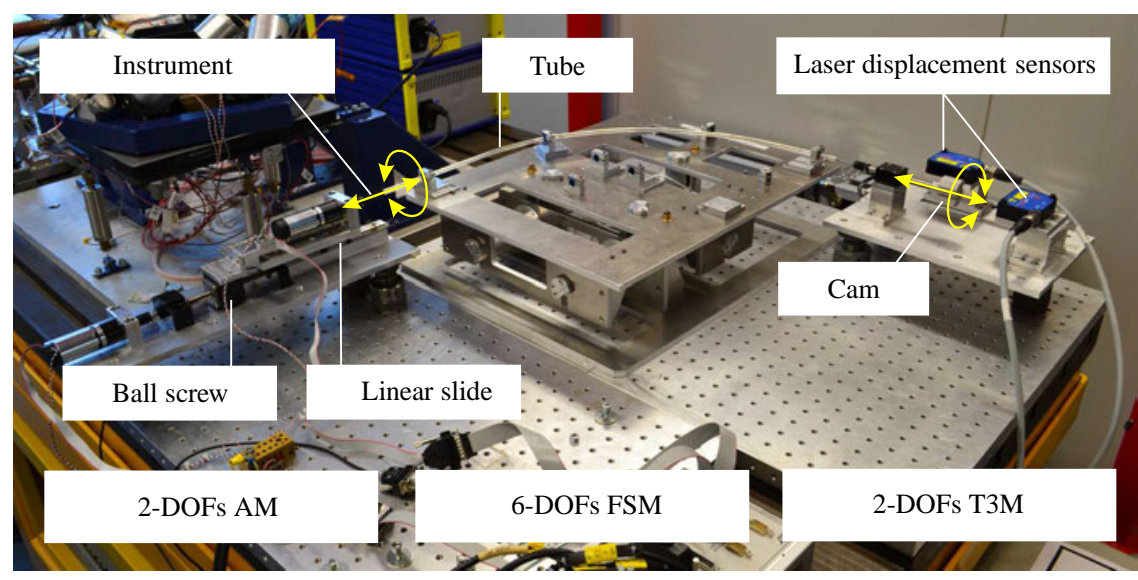

Fig. 3.11: Experimental set-up

on the top platform of the FSM. The flexible instrument was inserted through the tube and the two ends of the instrument were attached to the AM and the T3M for the actuation and measurement of the two DOFs respectively. The $\mathrm{AM}$ and the T3M were aligned to the entry and exit of the tube.

\subsection{Design Evaluation}

Table 3.1 shows the design specifications of the three modules-AM, FSM and T3M. The designed set-up has to be evaluated for the given specifications. First, the AM and the T3M were connected directly through a coupling for a combined evaluation as shown in Fig. 3.12. Next, the FSM was evaluated and characterized separately.

\subsubsection{Evaluation of the AM and the T3M}

We wanted to evaluate the following parameters of the AM and the T3M:

1. Translation of the AM

(a) Resolution

(b) Error due to gear backlash

2. Rotation of the AM

(a) Resolution

(b) Error due to gear backlash 


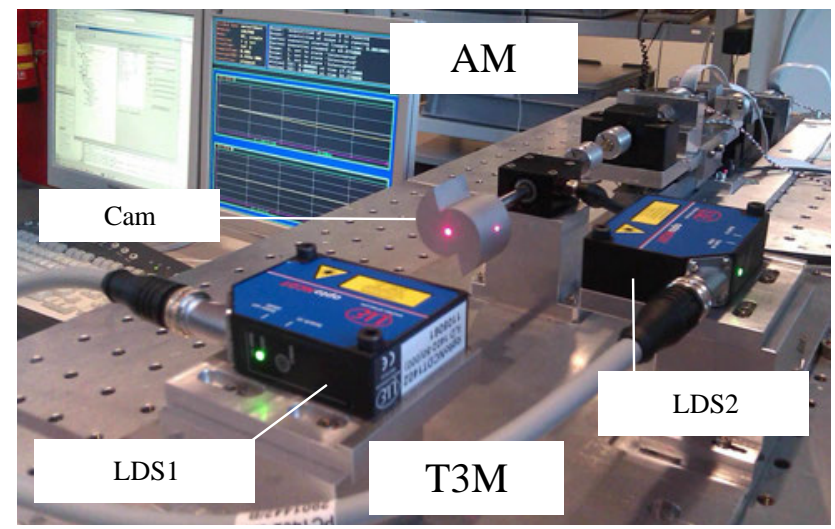

Fig. 3.12: The $A M$ and the $T 3 M$ were connected directly for the design evaluation

3. Translation measurement of the T3M

(a) Resolution

(b) Sensitivity of the LDS1

(c) Error due to the non-linearity of the LDS1

4. Rotation measurement of the $\mathrm{T} 3 \mathrm{M}$

(a) Resolution

(b) Sensitivity of the LDS2

(c) Error due to the non-linearity of the LDS2

The measurement of these parameters were performed through several experiments which are explained in the following sections.

\section{Resolution of the AM}

The translation axis of the AM has an encoder of $N_{p}=1000$ pulses per revolution and the DAQ acquires the signal through a quadrature encoder input. Including the pitch of the ball screw $p$ and the gear ratio $n_{g}$, the resolution based on the encoder resolution is, therefore, given by:

$$
\Delta x_{\text {res }}=\frac{p}{4 N_{p} n_{g}} .
$$

For $p=2 \mathrm{~mm}$ per revolution, and $n_{g}=4.38$, the resolution, thus calculated, is $0.1 \mu \mathrm{m}$. 
Chapter 3. Design of an Experimental Set-up

The rotation axis of the AM has also the encoder of 1000 pulses per revolution and the DAQ acquires the signal through the quadrature encoder input. The gear ratio is 19.22. Therefore, the resolution based on the encoder resolution is 0.005 degree.

However, the actual resolution is always marred by the friction in the system. The resolution of the AM in translation and rotation, mentioned in Tab. 3.1, will be sufficient for the characterization of the flexible instrument and the validation experiments.

\section{Resolution of the T3M}

The resolutions of the $\mathrm{T} 3 \mathrm{M}$ for the translation and rotation measurement were obtained from static measurements of the LDS1 and LDS2. The static measurements give an indication of the level of sensor noise and the effect of selected bandwidth on the resolution. A sample rate of $1 \mathrm{kHz}$ was used for the measurement. Figure 3.13 shows the static error measurement of the LDS1 and LDS2 when the cam was in the measurement range of both the sensors. A rather noisy signal was observed in both the cases. The power spectral density of the data showed high power in the frequency range, $100-150 \mathrm{~Hz}$. In order to reduce the sensor noise, a $2^{\text {nd }}$ order low-pass digital Butterworth filter was designed with cut-off frequency of $45 \mathrm{~Hz}$. The data were filtered off-line, forward and backward in time to achieve zero-phase digital filtering [35]. The static error was calculated by subtracting the mean value from the filtered data. The plots of the static error after filtering were also included in Fig. 3.13 for both sensors. We observed that the sensor noise was reduced considerably after filtering.

Static measurements were taken for the different positions in the measurement range to see if the error is same over the measurement range. Three different positions along the translation axis were considered—start, middle, and end of the measurement range. Output voltage from the LDS was measured, and the RMS value of the error was calculated from the standard deviation. The RMS values were calculated for both unfiltered and filtered data for both the LDS1 and LDS2. For LDS2 also, the static measurements were taken at three different positions in the measurement range. The sensitivity of each LDS was calculated from the change in the output voltage corresponding to the change in the input displacement (explained later in Section 3.4.1 and Section 3.4.1). After dividing with the sensitivity of the LDS, we obtained the error in the displacement measurement. Table 3.3 shows the result of the static error measurement taken from both the sensors. The error was reduced approximately 

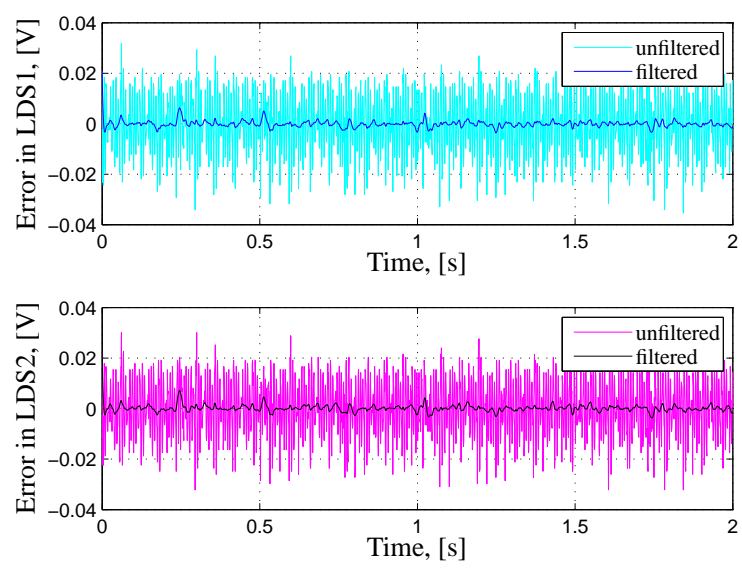

Fig. 3.13: Static error measurement of the LDS1 and LDS2

Table 3.3: Static error measurement of the LDS

\begin{tabular}{l|l|l|l|l}
\hline \multirow{2}{*}{ LDS1 } & \multicolumn{2}{|c|}{ Unfiltered } & \multicolumn{2}{c}{ Filtered } \\
\cline { 2 - 5 } & RMS value & Error & RMS value & Error \\
\hline Start & $8 \mathrm{mV}$ & $0.10 \mathrm{~mm}$ & $0.9 \mathrm{mV}$ & $11 \mu \mathrm{m}$ \\
Middle & $11 \mathrm{mV}$ & $0.14 \mathrm{~mm}$ & $1.2 \mathrm{mV}$ & $15 \mu \mathrm{m}$ \\
End & $11.5 \mathrm{mV}$ & $0.14 \mathrm{~mm}$ & $1.1 \mathrm{mV}$ & $14 \mu \mathrm{m}$ \\
\hline LDS2 & \multicolumn{4}{|l}{} \\
\hline Start & $10.6 \mathrm{mV}$ & $1.0 \mathrm{deg}$ & $1.4 \mathrm{mV}$ & $0.13 \mathrm{deg}$ \\
Middle & $10.4 \mathrm{mV}$ & $0.9 \mathrm{deg}$ & $1.2 \mathrm{mV}$ & $0.11 \mathrm{deg}$ \\
End & $10.5 \mathrm{mV}$ & $1.0 \mathrm{deg}$ & $1.4 \mathrm{mV}$ & $0.13 \mathrm{deg}$ \\
\hline
\end{tabular}

9 times after the filtering. The errors in the translation and rotation measurement are about $15 \mu \mathrm{m}$ and 0.15 degree respectively. Therefore, the resolution will be also of the same order. However, the resolution can be better or worse depending on the bandwidth chosen. For our application, a cut-off frequency of $45 \mathrm{~Hz}$ will be sufficient to capture the characteristic behavior of the flexible instrument.

\section{Sensitivity measurement of the LDS1}

For the sensitivity measurement of the LDS1, the output voltage from the LDS1 was obtained for the input displacement of the AM translation. The input displacement was obtained from the encoder reading. Figure 3.14 shows 

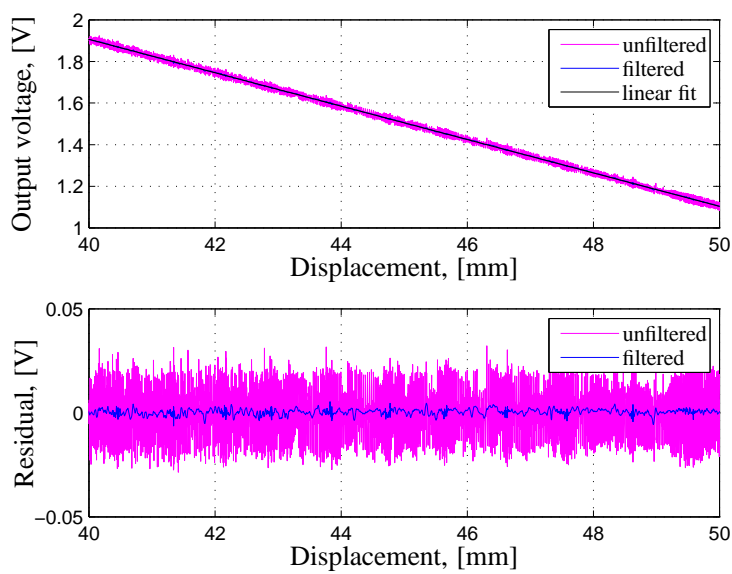

Fig. 3.14: Output voltage from the LDS1 for the translation measurement of the cam

the plot of the output voltage from the LDS1 for the translation measurement. A linear fit was applied to the data. The slope of the linear fit gives the sensitivity value which was $0.0803 \mathrm{~V} / \mathrm{mm}$.

The residual is calculated by subtracting the fitted data from the measurement data. The residual plot is also shown in Fig. 3.14. The error was calculated from the RMS value of the residuals. The RMS value of $11 \mathrm{mV}$ was obtained which was of the same order as of the sensor noise. The data were filtered off-line using the zero-phase digital filtering as explained in Section 3.4.1. The plot of the residuals of the filtered data from the linear fit was also shown in the figure. The error, thus obtained, was $1.3 \mathrm{mV}$. The error was reduced more than 8 times after the filtering. The error in the translation measurement due to the non-linearity of the sensor, obtained by dividing with the sensitivity of the LDS1, was $16 \mu \mathrm{m}$.

\section{Sensitivity measurement of the LDS2}

Similarly, for the sensitivity measurement of the LDS2, the output voltage from the sensor was obtained for the input displacement of the AM rotation. Figure 3.15 shows the plot of voltage output from the LDS2 for the rotation measurement. A linear fit was obtained for every 180 degree rotation of the cam, assuming the two lobes of the cam were identical. The sensitivity of the LDS2, thus obtained from the slope of the plot, was $0.0111 \mathrm{~V} / \mathrm{deg}$.

Figure 3.16 shows the residual plot of the LDS2 measurement from the linear fit. The data were filtered off-line by applying the zero-phase digital filter- 


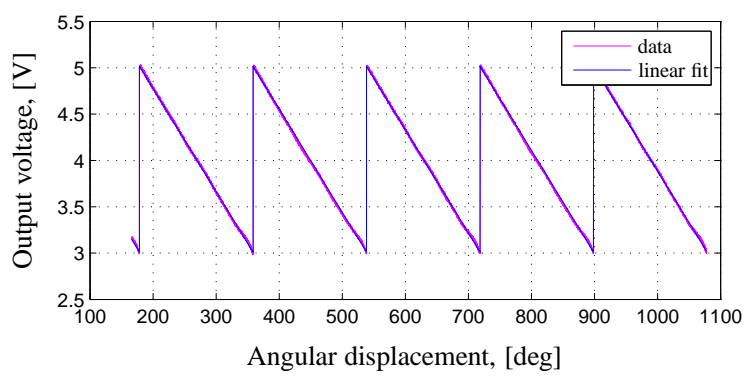

Fig. 3.15: Output voltage from the LDS2 for continuous rotation of the cam

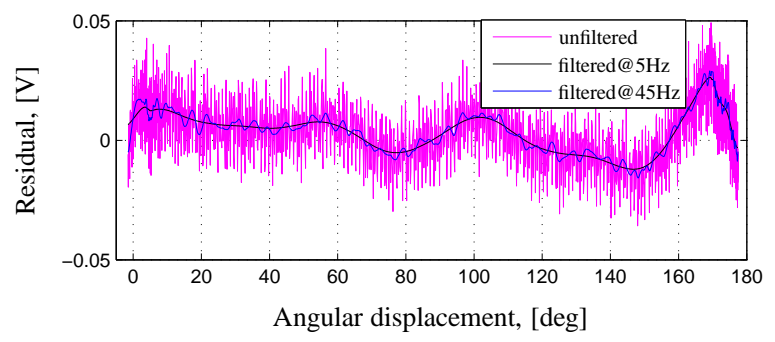

Fig. 3.16: Residual plot of the LDS measurement for rotation after a linear fit

ing as explained in Section 3.4.1 at a cut-off frequency of $5 \mathrm{~Hz}$ to get the shape of the cam profile. The data were filtered for a cut-off frequency of $45 \mathrm{~Hz}$ also for the error measurement. The figure also shows the plots of the residuals of the filtered data from the linear fit. The RMS value of the residuals gives the error because of the non-linearity of the cam. The RMS value of the error, thus obtained, was $9.2 \mathrm{mV}$. The corresponding error in angle measurement was 0.84 degree. The linearity of the cam is, therefore, $0.5 \%$. However, the error in the measurement can be further improved by using the look-up table for the cam.

Figure 3.17 shows the residual plots of the unfiltered and filtered data (at $45 \mathrm{~Hz}$ ) from the filtered data (at $5 \mathrm{~Hz}$ ) corresponding to the cam profile. These residual plots provide the error if the cam profile is taken into consideration. The RMS values for the unfiltered and filtered data were $10.6 \mathrm{mV}$ and $2.3 \mathrm{mV}$ respectively, and the corresponding errors in the angular measurement are 0.96 degree and 0.21 degree respectively. 


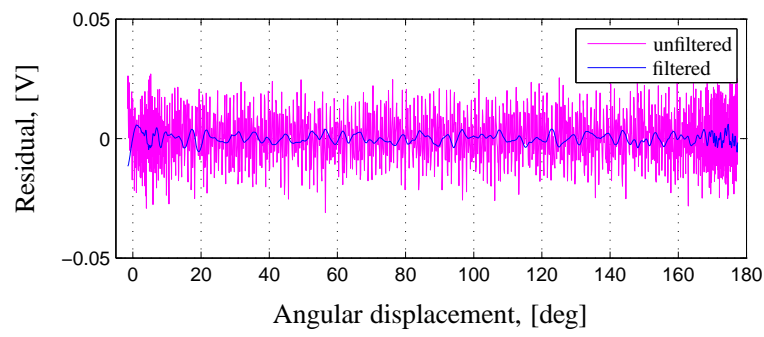

Fig. 3.17: Residual plot of the LDS measurement for rotation after subtracting the filtered data corresponding to the cam profile

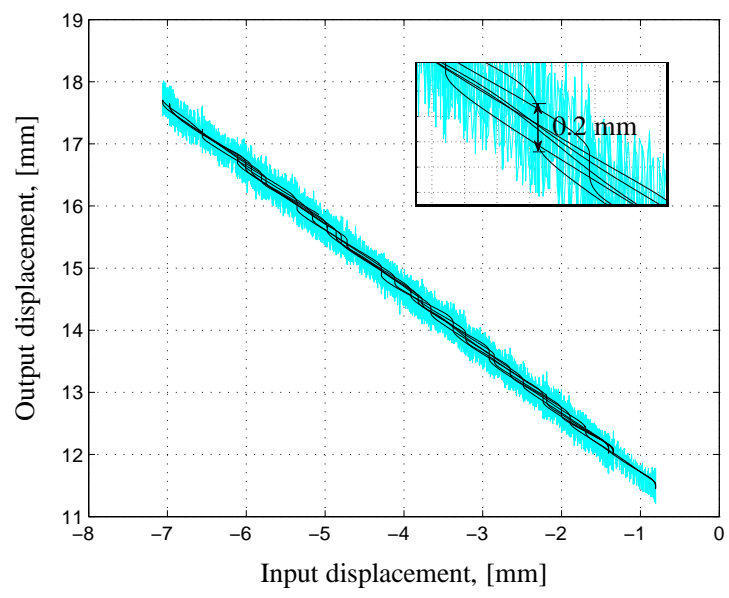

Fig. 3.18: Output displacement measured by the LDS1 compared with the input displacement measured from the encoder, showing gear backlash in translation

\section{Backlash measurement in the AM translation}

For the backlash measurement in the translation axis of the AM, the displacement measurement of the LDS1 was compared with the encoder reading corresponding to the translation, while the AM was moving in both directions along the translation axis. The input motion was given by rotating the coupling which connects the gear shaft to the ball screw. As the coupling was rotated, the ball screw was already moving, but the motor encoder was not moving because of the clearance in the gear box. Figure 3.18 shows the plot of the unfiltered and filtered data. The data were filtered using the the zero-phase digital filtering as explained in Section 3.4.1. A backlash of $0.2 \mathrm{~mm}$ was observed in the translation axis. 


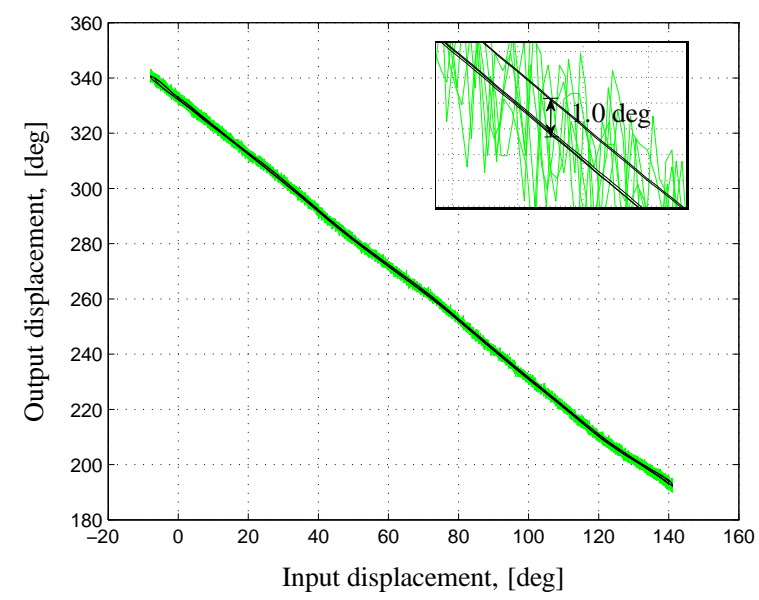

Fig. 3.19: Output displacement measured by the LDS2 compared with the input displacement measured from the encoder, showing gear backlash in rotation

\section{Backlash measurement in the AM rotation}

Similarly, for the backlash measurement in the rotation axis of the AM, the cam was rotated in both directions by rotating the coupling which connects the gear shaft with the T3M (as shown in Fig. 3.12). The displacement measurement of the LDS2 was compared with the encoder measurement along the rotation axis. Figure 3.19 shows the plot of the unfiltered and filtered data. The data were filtered using the the zero-phase digital filtering as explained in Section 3.4.1. The backlash of 1.0 degree was observed due to the clearance in the gear box.

\subsubsection{Evaluation of the FSM}

The FSM was designed to measure external forces and moments acting on the top floating plate. The designed module was calibrated by applying forces at known locations. If an external force and moment $\mathbf{F}_{e x t}$ is applied at point $P$ on the floating platform, the load cells attached to the base of six wire flexures will get loaded. Figure 3.20 illustrates the loading configuration. The equivalent force $\mathbf{F}_{x}$ acting at the origin $O$ can be expressed as:

$$
\mathbf{F}_{x}=B \mathbf{F}_{\text {ext }}
$$




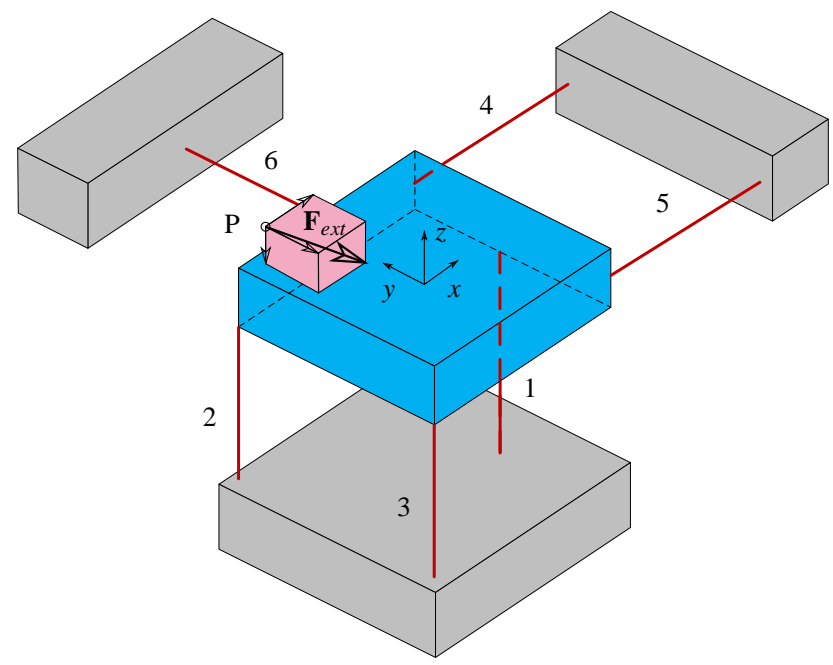

Fig. 3.20: Loading configuration showing external load applied on the top floating plate of the FSM at point $\mathrm{P} . \mathbf{F}_{\text {ext }}$ includes the external forces and moments.

or,

$$
\left[\begin{array}{c}
F_{x} \\
F_{y} \\
F_{z} \\
M_{x} \\
M_{y} \\
M_{z}
\end{array}\right]=\left[\begin{array}{rrrrrr}
1 & 0 & 0 & 0 & 0 & 0 \\
0 & 1 & 0 & 0 & 0 & 0 \\
0 & 0 & 1 & 0 & 0 & 0 \\
0 & -z & y & 1 & 0 & 0 \\
z & 0 & -x & 0 & 1 & 0 \\
-y & x & 0 & 0 & 0 & 1
\end{array}\right]\left[\begin{array}{c}
F_{x^{\prime}} \\
F_{y^{\prime}} \\
F_{z^{\prime}} \\
M_{x^{\prime}} \\
M_{y^{\prime}} \\
M_{z^{\prime}}
\end{array}\right]
$$

where $(x, y, z)$ are the coordinates of the point $P$ with respect to the origin. $F_{x^{\prime}}$, $F_{y^{\prime}}$, and $F_{z^{\prime}}$ are the forces, and $M_{x^{\prime}}, M_{y^{\prime}}$, and $M_{z^{\prime}}$ are the moments acting at point $P$ along $x y z$-directions respectively.

$\mathbf{F}_{x}$ can be further transformed into a force vector $\mathbf{F}_{n}$, consisting of six force components along the wire flexures, as expressed in Eqn. 3.2.

If $S$ is the sensitivity matrix of the load cells of the FSM, the voltage output of the load cells $\mathbf{V}_{n}$ is related to the force vector $\mathbf{F}_{n}$ as:

$$
\mathbf{V}_{n}=S \mathbf{F}_{n}
$$


or,

$$
\left[\begin{array}{l}
V_{1} \\
V_{2} \\
V_{3} \\
V_{4} \\
V_{5} \\
V_{6}
\end{array}\right]=\left[\begin{array}{llllll}
s_{11} & s_{12} & s_{13} & s_{14} & s_{15} & s_{16} \\
s_{21} & s_{22} & s_{23} & s_{24} & s_{25} & s_{26} \\
s_{31} & s_{32} & s_{33} & s_{34} & s_{35} & s_{36} \\
s_{41} & s_{42} & s_{43} & s_{44} & s_{45} & s_{46} \\
s_{51} & s_{52} & s_{53} & s_{54} & s_{55} & s_{56} \\
s_{61} & s_{62} & s_{63} & s_{64} & s_{65} & s_{66}
\end{array}\right]\left[\begin{array}{c}
F_{1} \\
F_{2} \\
F_{3} \\
F_{4} \\
F_{5} \\
F_{6}
\end{array}\right]
$$

where $s_{i j}$ is the sensitivity of the $i^{\text {th }}$ load cell because of the $j^{\text {th }}$ component of the force $\mathbf{F}_{n}$. If the wire flexures have only longitudinal stiffness $k_{a}$ and negligible lateral stiffness $k_{b}$, the force component $F_{n}$ will directly act on the $n^{\text {th }}$ load cell. Therefore, for larger ratio of stiffnesses $k_{a} / k_{b}$, the sensitivity matrix $S$ will be a diagonal matrix, and the diagonal terms will be the individual sensitivities of the load cells. However, because of the non-zero lateral stiffness (also called the parasitic stiffness) of the wire flexures, there will be resultant forces acting on the other load cells arising from the force acting along one of the load cells. This results into non-zero off-diagonal terms. The inaccuracies in the fabrication of the mechanical components and their assembly will also contribute to non-zero off-diagonal terms.

The evaluation of the FSM requires calculation of the following parameters:

1. Resolution of the load cells

2. Sensitivity of the load cells and the FSM

3. Error measurement of the FSM

The measurement of these parameters were performed through several experiments which are explained in the following sections.

\section{Resolution of the load cells}

Static measurements were taken from the load cells to obtain the sensor noise and the resolution. The output voltage from the six load cells was measured without an external load applied on the FSM. The loading on the load cells was from the weight of the top floating platform and the load due to the initial deflections along the compliant directions of the wire flexures.

Table 3.4 shows the static error measurement of the load cells. The RMS value of the error was calculated for each load cell for both unfiltered and filtered data. The filtered data were obtained by the zero-phase digital filtering as explained in Section 3.4.1 for a cut-off frequency of $45 \mathrm{~Hz}$. The corresponding 
Chapter 3. Design of an Experimental Set-up

Table 3.4: Static measurement of the load cells

\begin{tabular}{l|l|c|c|c|c|c|c}
\hline \multicolumn{2}{c|}{ Load cells } & 1 & 2 & 3 & 4 & 5 & 6 \\
\hline \multirow{2}{*}{ Unfiltered } & RMS value $(\mathrm{mV})$ & 3.3 & 2.9 & 3.1 & 2.9 & 2.8 & 2.9 \\
\cline { 2 - 8 } & Error $(\mathrm{mN})$ & 15.3 & 13.4 & 14.3 & 13.7 & 13.7 & 13.7 \\
\hline \multirow{2}{*}{ Filtered (at 45 Hz) } & RMS value $(\mathrm{mV})$ & 1.9 & 0.9 & 1.0 & 1.2 & 0.9 & 0.8 \\
\cline { 2 - 8 } & Error (mN) & 8.8 & 4.2 & 4.6 & 5.9 & 4.3 & 3.8 \\
\hline
\end{tabular}

error in force measurement was obtained by dividing with the individual sensitivity of the load cell which will be explained later in Section 3.4.2. The error in force measurement, thus calculated, was of the order of $9 \mathrm{mN}$ after filtering. Therefore, the resolution of the load cells is $9 \mathrm{mN}$ at $45 \mathrm{~Hz}$.

\section{Sensitivity of the load cells and the FSM}

For the sensitivity measurement of the load cells and the FSM, the output voltage of the load cells were measured by applying different loads at various locations along different directions. For a set of $\mathrm{N}$ measurements using Eqn. 3.11, the output voltage and the force components along the load cells for different external loads applied can be written as:

$$
\left[\begin{array}{llll}
\mathbf{V}_{n, 1} & \mathbf{V}_{n, 2} & \cdots & \mathbf{V}_{n, N}
\end{array}\right]=S\left[\begin{array}{llll}
\mathbf{F}_{n, 1} & \mathbf{F}_{n, 2} & \cdots & \mathbf{F}_{n, N}
\end{array}\right]
$$

or,

$$
\left[V_{n N}\right]=S\left[F_{n N}\right]
$$

where $\mathbf{V}_{n, j}$ and $\mathbf{F}_{n, j}$ are the output voltage from the load cells $\mathbf{V}_{n}$ and the force components $\mathbf{F}_{n}$ for the $j^{\text {th }}$ measurement. $\mathbf{F}_{n}$ is calculated for each external load applied $\mathbf{F}_{\text {ext }}$. Using Eqn. 3.2 and Eqn. 3.10, we have

$$
\mathbf{F}_{n}=A^{-1} B \mathbf{F}_{\text {ext }} .
$$

The individual sensitivities of the load cells $S_{i}$ were obtained directly by plotting the output voltage $V_{i N}$ with respect to the load $F_{i N}$ acting along the $i^{t^{t h}}$ load cell. Figure 3.21 shows the plot of the output voltage of the load cells. The sensitivities of the individual load cells were obtained from the slopes of the linear fit and summarized in Tab. 3.5. The off-diagonal elements cannot be obtained by this method.

Alternatively, the full sensitivity matrix $S$ of the load cells of the FSM can be calculated from Eqn. 3.12 using the pseudo-inverse as

$$
S=\left[\begin{array}{llll}
\mathbf{V}_{n, 1} & \mathbf{V}_{n, 2} & \cdots & \mathbf{V}_{n, N}
\end{array}\right]\left[\begin{array}{llll}
\mathbf{F}_{n, 1} & \mathbf{F}_{n, 2} & \cdots & \mathbf{F}_{n, N}
\end{array}\right]^{+}
$$




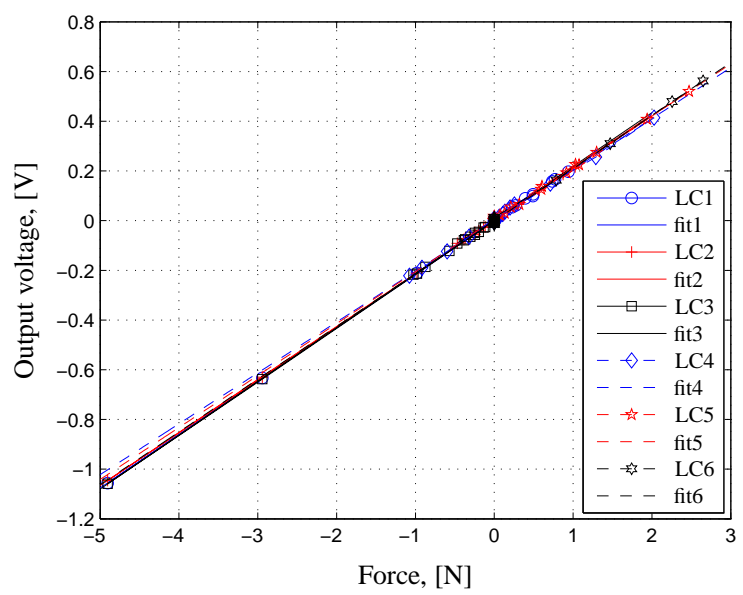

Fig. 3.21: Output voltage of the load cells for the forces acting along the load cells

Table 3.5: Individual sensitivity of the load cells

\begin{tabular}{l|l|l|l|l|l|l}
\hline & $S_{1}$ & $S_{2}$ & $S_{3}$ & $S_{4}$ & $S_{5}$ & $S_{6}$ \\
\hline Sensitivity (V/N) & 0.215 & 0.213 & 0.216 & 0.205 & 0.209 & 0.212 \\
\hline
\end{tabular}

or,

$$
S=\left[V_{n N}\right]\left[F_{n N}\right]^{+}
$$

where $\left[F_{n N}\right]^{+}$is the pseudo-inverse of the matrix. The sensitivity matrix thus obtained is

$$
S=\left[\begin{array}{rrrrrr}
0.2152 & 0.0010 & -0.0006 & -0.0008 & -0.0003 & 0.0004 \\
0.0008 & 0.2135 & 0.0009 & 0.0010 & 0.0033 & -0.0012 \\
-0.0001 & -0.0003 & 0.2162 & 0.0004 & 0.0026 & 0.0007 \\
-0.0018 & 0.0008 & 0.0010 & 0.2035 & 0.0068 & -0.0039 \\
0.0001 & 0.0003 & -0.0036 & 0.0039 & 0.2108 & -0.0005 \\
0.0015 & -0.0005 & 0.0017 & -0.0086 & 0.0061 & 0.2067
\end{array}\right]
$$

The diagonal and off-diagonal elements can be obtained simultaneously. We also observed that the diagonal elements are at least 24 times higher than the off-diagonal elements. It was also observed that the diagonal elements are almost identical to the individual sensitivities of the load cells. 

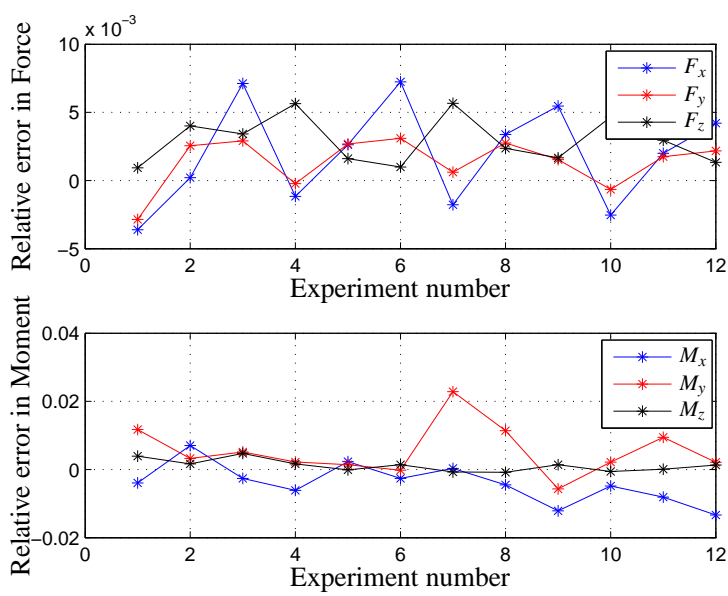

Fig. 3.22: Relative error in force and moment measurement

\section{Error measurement of the FSM}

The forces acting on FSM are measured in $x y z$-coordinates. Therefore, the error in measurement should be expressed in terms of three forces and three moments along xyz-coordinates. The estimated force $\hat{\mathbf{F}}_{x}$ can be calculated from the output voltage readings of the load cells $\mathbf{V}_{n}$ using Eqn. 3.2 and Eqn. 3.11 as:

$$
\hat{\mathbf{F}}_{x}=A S^{-1} \mathbf{V}_{n}
$$

$\mathbf{F}_{x}$ can be directly obtained from the external load applied $\mathbf{F}_{\text {ext }}$ using Eqn. 3.10. The error can be calculated from $\mathbf{F}_{x}$ and $\hat{\mathbf{F}}_{x}$.

The relative errors in force and moment measurements were calculated for a new set of experiments where known loads were applied on the top floating plate of the FSM. Figure 3.22 shows the plot of relative error in force and moment measurement. The error obtained in the force and moment measurements are less than $0.8 \%$ and $2.5 \%$ respectively.

\subsection{Discussion}

The AM has a translation resolution of $0.1 \mu \mathrm{m}$ and a rotation resolution of 0.005 degree based on the encoder resolution. However, the actual resolution is always marred by the friction in the system. However, the resolution of the AM at least exceeds the resolution of the T3M, which is of the order of $15 \mu \mathrm{m}$ in translation and 0.15 degree in rotation. This will be sufficient for 
the characterization of the flexible instrument and the validation experiments. The largest errors of the AM are because of the gear backlash. The backlash observed in translation and rotation is $0.2 \mathrm{~mm}$ and 1.0 degree respectively. The motion hysteresis observed in the simulation of flexible instruments were of the order of $2 \mathrm{~mm}$ in translation [26,31]. The motion hysteresis in rotation is expected to be of the order of 10 degree. However, if required the gear can be replaced or preloaded. The AM can achieve slow speeds of the order of $0.2 \mathrm{~mm} / \mathrm{s}$ in translation and $0.1 \mathrm{rev} / \mathrm{s}$ in rotation, which can be useful for the study of stick-slip behavior of the instrument. The AM can achieve a higher speed of $30 \mathrm{~mm} / \mathrm{s}$ in translation and $4 \mathrm{rev} / \mathrm{s}$ in rotation, which can be used to generate a combination of translation and rotation motion.

We used a low pass filter with a cut-off frequency of $45 \mathrm{~Hz}$ for improving the resolution of the T3M. This enables filtering any noise due to the power supply of $50 \mathrm{~Hz}$. At the same time, it is high enough to perform dynamic measurements. However, the stick-slip behavior of flexible instruments due to friction may require higher bandwidth. The error in rotation measurement due to the non-linearity of the cam is of the order of 1.0 degree. However, the error was reduced to 0.2 degree if the cam profile was taken into consideration. The specifications of the T3M are comparable to the design specifications.

The load cells of the FSM have a measurement range of $50 \mathrm{~N}$ with a resolution of $9 \mathrm{mN}$ at $45 \mathrm{~Hz}$. The error in the measurements of force and moment are less than $0.8 \%$ and $2.5 \%$ respectively. These values allow accurate measurement of the forces a flexible instrument exerts on the tube.

The designed experimental set-up is used to validate the simulation results from the flexible multibody model of a flexible instrument inside a curved rigid tube. The achieved specifications of the three modules-AM, FSM and $\mathrm{T} 3 \mathrm{M}$ - enabled us to provide actuation in translation and rotation along the longitudinal axis of the instrument at the proximal end, to measure the interaction forces, and to measure the translation and rotation of the distal end of the instrument. However, the details of the experiments are beyond the scope of this paper and can be referred to [25].

\subsection{Conclusion}

We designed the experimental set-up based on the design requirements necessary to validate the flexible multibody model and to characterize the flexible instruments inside an endoscope. The Actuation Module provides the actuation in axial and twist direction from the proximal end of the flexible instru- 
Chapter 3. Design of an Experimental Set-up

ment. Although there is a backlash of $0.2 \mathrm{~mm}$ and 1.0 degree in the module, the motion hysteresis in the case of a flexible instrument is at least 10-times higher than the error due to backlash. The Tip Motion Measurement Module achieves contactless measurement of the distal end motion in axial and twist direction via an air bearing guided cam. Off-line filtering helps to improve the resolution to $15 \mu \mathrm{m}$ and 0.15 degree. The Force Sensing Module measures the force and moment exerted by the flexible instrument on the curved tube which is fixed to the floating platform. The force resolution of $9 \mathrm{mN}$ and the force and moment error of $0.8 \%$ and $2.5 \%$ respectively are within the specifications. The three modules of the set-up conform to the design objectives. The experimental set-up is used for the validation of the developed model of the flexible instrument inside a curved rigid tube.

\section{Acknowledgment}

The authors would like to thank Mr. L. Tiemersma for his contribution to the realization of the set-up, and Dr. Johannes van Dijk for his help in XPC and control.

This research is funded with the TeleFLEX project, number PID07038, by the Dutch Department of Economic Affairs, Agriculture and Innovation and the Province of Overijssel within the PIDON initiative. 


\section{Flexible Multibody Modelling of a Surgical Instrument Inside an ENDOSCOPE}

The implementation of flexible instruments in surgery necessitates high motion and force fidelity, and good controllability of the tip. However, the positional accuracy and the force transmission of these instruments are jeopardized by the friction, the clearance and the inherent compliance of the instrument. The surgical instrument is modeled as a series of interconnected spatial beam elements. The endoscope is modeled as a rigid curved tube. Stiffness, damping, and friction are defined to calculate the interaction between the instrument and the tube. The effect of various parameters on the motion and force transmission behavior was studied for the axially-loaded and no-load cases. The simulation results showed a deviation of $1.8 \%$ in the estimation of input force compared with the analytical capstan equation. The experimental results showed a deviation of the order of $1.0 \%$. The developed flexible multibody model is able to demonstrate the characteristic behavior of the flexible instrument for both translational and rotational input motion for a given set of parameters. The developed model will help us to study the effect of various parameters on the motion and force transmission of the instrument.

J.P. Khatait, D.M. Brouwer, J.P. Meijaard, R.G.K.M. Aarts, \& J.L. Herder (2013). Flexible multibody modeling of a surgical instrument inside a curved endoscope, Journal of Computational and Nonlinear Dynamics, draft submitted, under review. 


\subsection{Introduction}

Minimally Invasive Surgery (MIS) has greatly reduced the unnecessary damage and trauma to healthy tissues, leading to faster recovery, reduced infection rates, and reduced post-operative complications. Most of the limitations imposed by conventional laparoscopic systems are well addressed in modern surgical robotic systems by increasing dexterity, restoring proper hand-eye coordination, an ergonomic working position, and improving visualization. The state-of-the-art robotic surgery systems employ rigid instruments $[6,14,32]$. However, with conventional colonoscopy and with the emergence of Natural Orifice Transluminal Endoscopic Surgery (NOTES) and Single Incision Laparoscopic Surgery (SILS) procedures, the use of flexible instruments is inevitable.

As the flexible endoscopy continues to evolve more into a therapeutic tool and as the endoscopic procedures are becoming more invasive, the surgical instruments require complex manipulations $[7,57]$. The instrument tip needs to deliver motion and force with a required precision and accuracy. The motion and force transmission of these instruments are critical for achieving good surgical outcomes and need to be thoroughly researched. In this paper, an endoscope refers to a flexible endoscope typically used for the examination of gastrointestinal tract, for example, during colonoscopy and gastroscopy procedures. The instrument refers to the flexible instrument used for biopsy or for simple surgical procedures, which is fed through the access channel of the endoscope.

In an endoscope-like surgical system, the instrument is controlled from the proximal end. The friction between the instrument and the access channel of the endoscope introduces nonlinearities in motion transmission. This makes the estimation and the control of the instrument tip difficult and challenging in the absence of sensors at the distal end. This is further aggravated due to the limited stiffness of the instrument and the added clearance. Moreover, depending on the location of the surgical site, the shape of the endoscope also changes and can be very sinuous.

A thorough understanding of the flexible instrument behavior inside the access channel of the endoscope can lead to a proper design of the instrument and can eventually lead to improved control of the instrument tip for the desired motion or force. In our previous study [26,31], we set up a 2-D flexible multibody model to study the effect of friction and bending stiffness of the instrument on motion hysteresis. We studied the sliding behavior of the flexible instrument inside a curved endoscope in the presence of friction. However, the 
model was limited to planar cases and the model can address only the translational behavior. A 3-D flexible multibody model is required to address the dynamic behavior of a flexible instrument in rotation and translation. The objective of this paper is to set up a general 3-D flexible multibody model of the surgical instrument to study its translational and rotational behavior in a 3-D environment. In addition, we want to validate the model with experiments and an analytical model.

Firstly, a general 3-D flexible multibody model is set up for the instrument. The model of the curved tube is also defined. The contact force model is appropriately defined to include friction. The model of the flexible instrument and of the endoscope are explained in detail in Section 4.2. The contact force model is explained subsequently in Section 4.2.3. Secondly, various simulations are performed for both translation and rotation input motion at the proximal end. The developed 3-D model is firstly simulated for the insertion in a planar tube and is used to validate the 3-D model with the previously developed 2-D model [26]. The simulation results are in good agreement with the 2-D model and can be referred to [28]. The simulations for fine manipulation in translation in a curved tube are performed for different values of the coefficient of friction between the contacting surfaces. The simulations are performed for both the axially-loaded and no-load cases. The motion and force transmission is analyzed and compared with the capstan equation. The simulations for fine manipulation in rotation are also performed for different values of the coefficient of friction. The effect of different flexural rigidity along the principal axes of the instrument and the effect of the pre-curvature are analyzed on the motion transmission in rotation. The details of the simulation cases and the results are discussed in Section 4.3. Finally, various experiments are carried out with a standard stainless steel wire. The value of the coefficient of friction is estimated. The force transmission in translation is verified analytically. The simulation result for the estimated parameters is also compared with the experimental result. Experiments for rotation input motion are also carried out and compared with the simulation results. The various experiments for the model verification and for the estimation of different parameters are explained in Section 4.4. The discussion and conclusion follow in Section 4.5 and Section 4.6 respectively. 


\subsection{Modeling of a Flexible Surgical Instrument Inside a Curved Endoscope}

The modeling of a flexible surgical instrument to study its dynamic behavior inside the endoscope requires modeling of three key components- the surgical instrument, the endoscope, and the interaction between the two. The surgical instrument must be flexible to allow easy insertion into the curved endoscope channel. In general, the flexible instrument deforms mainly in the bending and torsional directions due to the loading conditions at the instrument tip, the motion and force input at the proximal end, and the interaction of the instrument with the endoscope. The model of the instrument incorporates all the deformations under different loading conditions. The finite element model of the instrument is further elaborated in Section 4.2.1.

The endoscope is also flexible so that it can be easily inserted inside a lumen of the human body. The endoscope conforms to the human anatomy and takes different geometric shapes. The endoscope is supported by the organs or the anatomy in contact. The endoscope is assumed to be rigid with respect to the instrument as the inertia and the stiffness of the instrument is much smaller than that of the endoscope. However, if the instrument tip interacts with the tissue and a higher force is involved in the manipulation, the endoscope deforms together with the human anatomy in contact. Nonetheless, as we are keen in understanding the characteristic behavior of the instrument in a curved endoscope, the scope is limited to the curved rigid endoscope. The model of the endoscope is explained further in Section 4.2.2.

A contact force model is defined to calculate the interaction between the instrument and the curved rigid tube. Contact with rigid surfaces introduces non-linearity into the system and can be computationally heavy to simulate. A contact model, comprising of the wall stiffness, damping and friction, is further elaborated in Section 4.2.3.

The model of the flexible instrument with the curved rigid tube is shown in Fig. 4.1. The origin of the global frame, $O$, is situated at the beginning of the tube and the initial tangential direction is the $X$-axis. The encircled number, (1), represents the $n^{\text {th }}$ beam element. The nodes are represented by the numbers.

\subsubsection{A Flexible Surgical Instrument as Interconnected Beam El- ements}

The surgical instrument is modeled as a series of interconnected flexible beam elements as available in the SPACAR program [21]. Each beam element has 


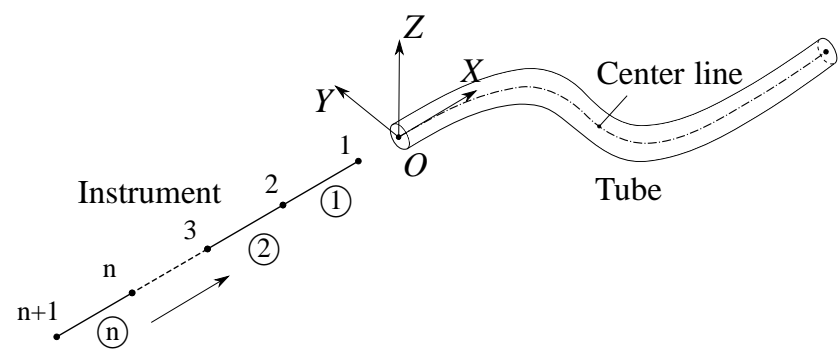

Fig. 4.1: Model of the instrument with the curved tube

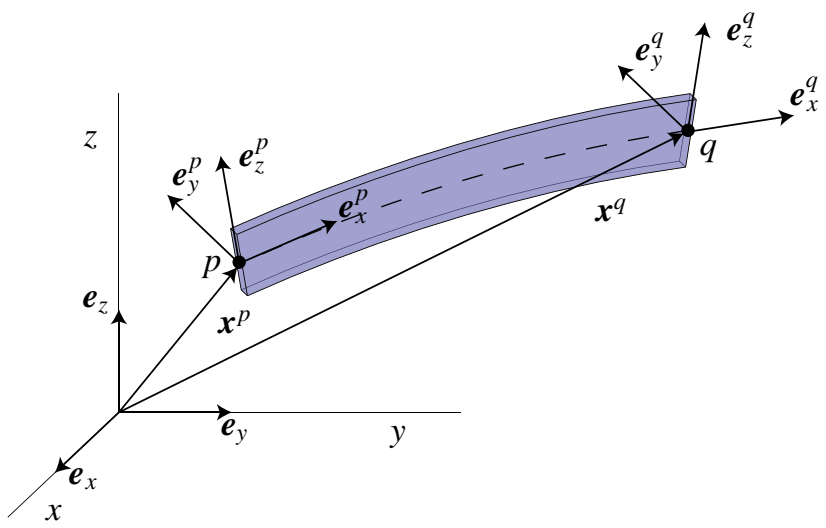

Fig. 4.2: The spatial flexible beam element

a node at either end. At each node six independent coordinates are definedthree translations and three rotations. Hence, the configuration of the element is determined by the position vectors $\boldsymbol{x}^{p}$ and $\boldsymbol{x}^{q}$, and the angular orientation of the orthonormal triads $\left[\boldsymbol{e}_{x}^{p}, \boldsymbol{e}_{y}^{p}, \boldsymbol{e}_{z}^{p}\right]$ and $\left[\boldsymbol{e}_{x}^{q}, \boldsymbol{e}_{y}^{q}, \boldsymbol{e}_{z}^{q}\right]$ rigidly attached to the end nodes $p$ and $q$, as shown in Fig. 4.2. In the undeformed state, the triads coincide with the axis $p-q$ and the principal axes of its cross-section. Therefore, the vector of nodal coordinates for the beam element is expressed as the two sets of Cartesian coordinates, $\boldsymbol{x}^{p}$ and $\boldsymbol{x}^{q}$, and two sets of Euler parameters, $\boldsymbol{\lambda}^{p}$ and $\lambda^{q}$, representing the orientation of the triads at the nodes $p$ and $q$

$$
\boldsymbol{x}_{\text {beam }}=\left[\begin{array}{llll}
\boldsymbol{x}^{p T}, & \lambda^{p T}, & \boldsymbol{x}^{q T}, & \lambda^{q T}
\end{array}\right]^{T} .
$$

The spatial beam element has a total of fourteen nodal coordinates with two constraint conditions for the Euler parameters. The number of degrees of freedom of the element as a rigid body is six. Consequently, the beam element 
has six deformation modes defined as [22]:

$$
\begin{aligned}
\text { elongation: } & \epsilon_{1}=\mathcal{D}_{1}(\boldsymbol{x})=l-l_{0}, \\
\text { torsion: } & \epsilon_{2}=\mathcal{D}_{2}(\boldsymbol{x})=l_{0}\left(\boldsymbol{e}_{z}^{p} \cdot \boldsymbol{e}_{y}^{q}-\boldsymbol{e}_{y}^{p} \cdot \boldsymbol{e}_{z}^{q}\right) / 2, \\
\text { bending: } & \epsilon_{3}=\mathcal{D}_{3}(\boldsymbol{x})=-l_{0} \boldsymbol{e}_{l} \cdot \boldsymbol{e}_{z}^{p}, \\
& \epsilon_{4}=\mathcal{D}_{4}(\boldsymbol{x})=l_{0} \boldsymbol{e}_{l} \cdot \boldsymbol{e}_{z}^{q}, \\
& \epsilon_{5}=\mathcal{D}_{5}(\boldsymbol{x})=l_{0} \boldsymbol{e}_{l} \cdot \boldsymbol{e}_{y}^{p}, \\
& \epsilon_{6}=\mathcal{D}_{6}(\boldsymbol{x})=-l_{0} \mathbf{e}_{l} \cdot \boldsymbol{e}_{y}^{q},
\end{aligned}
$$

where $l=\left\|\boldsymbol{x}^{q}-\boldsymbol{x}^{p}\right\|$ is the distance between the nodal points, $l_{0}$ is the reference length of the element, and $\boldsymbol{e}_{l}=\left(\boldsymbol{x}^{q}-\boldsymbol{x}^{p}\right) / l$ is the unit vector directed from node $p$ to node $q$. The first and second deformation modes, $\epsilon_{1}$ and $\epsilon_{2}$, describe the elongation and torsion of the element. The other four deformation modes, $\epsilon_{3}-\epsilon_{6}$, are related to the bending deformations of the element. The analytical functions $\mathcal{D}_{i}$ are called deformation functions.

\section{Kinematic Analysis}

The kinematic model is obtained by assembling the finite elements. The elements are connected at the common nodal points. The vector of nodal coordinates $\boldsymbol{x}$, thus formed, defines the configuration of the instrument. The vector $\boldsymbol{x}$ is, therefore, given by

$$
\boldsymbol{x}=\left[\begin{array}{llll}
x_{1} & x_{2} & \ldots & x_{n x}
\end{array}\right]^{T}
$$

where $n x$ represents the total number of nodal coordinates.

The vector of deformation modes $\boldsymbol{e}$ of all elements are then written as a vector function of the nodal coordinates

$$
e=\mathcal{D}(x) .
$$

Kinematic constraints are introduced by imposing conditions on both nodal coordinates $x_{i}$ and deformation mode coordinates $e_{i}$.

The configuration and the deformation state of the instrument is defined by the independent generalized coordinates $q_{i}$. The vector of all the independent generalized coordinates $\boldsymbol{q}$ is, therefore, called the vector of degrees of freedom (DOF). It includes the vector of independent generalized nodal coordinates $\boldsymbol{x}^{(m)}$ and the vector of independent deformation mode coordinates $\boldsymbol{e}^{(m)}$

$$
\boldsymbol{q}=\left[\begin{array}{l}
\boldsymbol{x}^{(m)} \\
\boldsymbol{e}^{(m)}
\end{array}\right] .
$$


The motion of the instrument is described as the function of the vector $\boldsymbol{q}$ of the independent generalized coordinates. The solution is expressed as

$$
\begin{aligned}
\boldsymbol{x} & =\mathcal{F}^{(x)}(\boldsymbol{q}), \\
\boldsymbol{e} & =\mathcal{F}^{(e)}(\boldsymbol{q}),
\end{aligned}
$$

where $\mathcal{F}^{(x)}$ and $\mathcal{F}^{(e)}$ are called the geometric transfer functions. The geometric transfer functions cannot be calculated explicitly from Eqn. (4.4), but they have to be determined numerically in an iterative way [21]. The velocity vectors and the acceleration vectors are obtained by differentiating Eqn. (4.6) successively with respect to time.

\section{Dynamic Analysis}

In a dynamic analysis, the equation of motion is solved first. The equations relate forces and torques to positions, velocities, and accelerations. The inertia properties of the concentrated and distributed mass of the elements are described by means of lumped and consistent mass matrices. The global mass matrix is obtained by assembling the lumped and consistent element mass matrices. The stiffness properties are also defined for the flexible elements by means of stiffness matrices relating stress resultants and deformations. The stiffness matrices are calculated using polynomial interpolations for the deformations of the element. A distributed mass along the center line and lumped moments of inertia at the nodes for the rotational inertia are defined. The detailed description of the element and its stiffness and inertia properties can be found in [40].

The equations of motion are derived using the principle of virtual power and the principle of d'Alembert. By making use of the geometric transfer function, Eqn. (4.6), the equations of motion are written as a set of second order ordinary differential equations for the independent generalized coordinates $\boldsymbol{q}$. For the detailed description and derivation of kinematic and dynamic analysis of elements, the readers can refer to $[20,63]$.

\section{Numerical Integration Methods}

The equations of motion are solved numerically using SPACAR program. It has a comprehensive list of integrators, which includes various explicit, implicit, and semi-implicit methods [41,53]. We used the explicit third-order Runge-Kutta method with the variable step-size. The step-size is selected 
based on an estimate for the truncation error. The small error bound guides the integration method towards choosing sufficiently small time steps needed for the stability [42]. A relative error bound of $1.0 \times 10^{-4}$ is chosen for the solution. However, this has to be chosen carefully as larger value leads to instability, but on the other hand a smaller value leads to longer computation time. For the stability of the various numerical integration methods and the limit to the step-size, the readers can refer to [41].

\section{Discussion}

Different kinds of finite elements are needed to model the complete surgical instrument. A hinge element is used to prescribe the input rotation motion at the proximal end of the instrument $[12,63]$. The entire length of the instrument is modeled by choosing multiple spatial beam elements. As the shape of the tube changes, the minimum number of the beam elements required to accurately model the instrument behavior also changes. The higher the curvature of the tube, the smaller is the required length of the beam element. Therefore, more beam elements are required for the same length of the instrument if the tube is more sinuous.

A length ratio $\left(l_{e} / R_{\min }\right)$ can be defined and used as a parameter to choose an optimum length of the beam element for a given shape of a curved tube. Here, $l_{e}$ is the element length and $R_{\min }$ is the minimum radius of curvature of the tube. As the instrument is always confined within the tube, this also ensures limited deformation within an individual element. This is also equal to the angle subtended by the element at the center of curvature. Moreover, more elements also mean high computational time required for the simulation. An element length, corresponding to $15^{\circ}$ angle subtended, has been used by ten Hoff for the study of rotation transmission behaviour of a flexible driveshaft [60]. This corresponds to the length ratio of 0.26 . Hakvoort used 12 elements to define the semicircular part of the curve to model a Coriolis massflow meter for the shape optimization [15]. We further investigated the effect of the length ratio on the sliding behavior of a flexible instrument inside a circular tube and observed that a length ratio smaller than 0.15 gives a sufficiently accurate result [26]. We used this as a basis in choosing the length of the beam element. However, the model has to be refined or coarsened depending on the accuracy or the computation time required. 


\subsubsection{An Endoscope as a Rigid Curved Tube}

The endoscope is modeled as a rigid curved tube of uniform circular crosssection. The shape of the tube is defined by the centerline of the tube. The centerline is defined by a straight line, a circular arc, a Bézier curve, or a combination of these. Different geometric shapes are connected together to define the entire length of the curved tube. The tangents at the joining end of the two sections are the same. A planar case was explained in [26,31], and can be referred to for the detailed description. However, for the 3-D case, the rigid curved tube has to be defined in the 3-D space and is explained in detail in this section. The kinematics of the contact between the instrument and the endoscope is explained in detail with respect to the geometric shape of the curve chosen to describe the section of the curved tube.

\section{Kinematics of the contact between the instrument and the endoscope}

As the instrument progresses inside the curved tube, the contact takes place at the nodes. The position of the interacting node is given by the position vector $\mathbf{P}_{o}$. The corresponding closest point on the center line of the tube is called the base point and denoted by the position vector $\mathbf{P}_{c}$. The tangent vector at $\mathbf{P}_{c}$ is represented by $\mathbf{P}_{c}^{\prime}$. Three orthogonal directions-radial, axial, and circumferential—are defined at the interacting node and denoted by the unit vectors $\mathbf{e}_{1}, \mathbf{e}_{2}$ and $\mathbf{e}_{3}$ respectively. The triad can be termed as the contact triad and expressed as

$$
\begin{aligned}
\mathbf{e}_{1} & =\frac{\left(\mathbf{P}_{o}-\mathbf{P}_{c}\right)}{\left\|\mathbf{P}_{o}-\mathbf{P}_{c}\right\|} \\
\mathbf{e}_{2} & =\frac{\mathbf{P}_{c}^{\prime}}{\left\|\mathbf{P}_{c}^{\prime}\right\|} \\
\mathbf{e}_{3} & =\mathbf{e}_{1} \times \mathbf{e}_{2} .
\end{aligned}
$$

Figure 4.3 shows the contact triad attached to the interacting node $\mathbf{P}_{o}$.

The calculation of the contact triad requires finding the position of the base point $\mathbf{P}_{c}$ on the centerline and the tangent vector $\mathbf{P}_{c}^{\prime}$ at the base point. The calculation of $\mathbf{P}_{c}$ and $\mathbf{P}_{c}^{\prime}$ are explained here for a Bézier tube and a circular tube.

Bézier Tube A 3-D cubic Bézier curve is defined by a set of four control points. The first and last control points, $\mathbf{P}_{1}$ and $\mathbf{P}_{4}$, coincide with the endpoints $\mathbf{P}_{s}$ and $\mathbf{P}_{e}$ of the curve. The shape of the curve is determined by the interior 


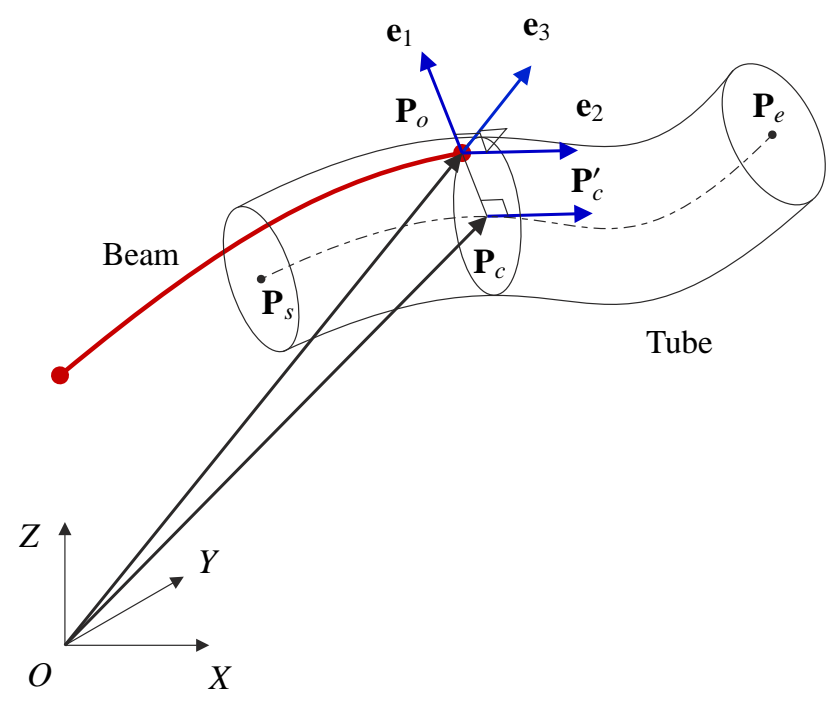

Fig. 4.3: Contact triad at the interacting node $\mathbf{P}_{o}$ of the beam when in contact with the Bézier tube

control points, $\mathbf{P}_{2}$ and $\mathbf{P}_{3}[9,11]$. A part of the 3-D tube can be defined by selectively choosing the control points.

Figure 4.3 shows the centerline of the curved tube defined by a Bézier curve. $\mathbf{P}(u)$ is the location of a point on the Bézier curve depending on the parameter $u$ for $0 \leq u \leq 1$. The tangent vector $\mathbf{P}_{c}^{\prime}$ is given by the parametric derivative of the curve at point $\mathbf{P}_{c}$. The starting point $\mathbf{P}_{s}$ of the curve corresponds to $\mathbf{P}(0)$ and the end point $\mathbf{P}_{e}$ to $\mathbf{P}(1)$. The vector $\left(\mathbf{P}_{o}-\mathbf{P}_{c}\right)$ is perpendicular to the tangent vector $\mathbf{P}_{c}^{\prime}$. The point $\mathbf{P}_{c}$ is obtained by solving

$$
\left(\mathbf{P}_{o}-\mathbf{P}_{c}\right) \cdot \mathbf{P}_{c}^{\prime}=0
$$

for $0 \leq u \leq 1$. The value of $u$, satisfying Eqn. (4.8), locates the point $\mathbf{P}_{c}$ and the tangent vector $\mathbf{P}_{c}^{\prime}$. The detailed description of the Bézier curve and the calculation of the base point and the tangent vector can be referred to [26,31]. Figure 4.3 shows the contact triad at the interacting node $\mathbf{P}_{o}$ inside the Bézier tube.

Circular Tube A circular tube is defined by an arc as the centerline of the tube. The arc is defined by the radius $R$, the center $\mathbf{P}_{c o}$, and the unit vector $\mathbf{e}_{b}$ normal to the plane containing the arc. Figure 4.4 shows the configuration of the circular tube. 


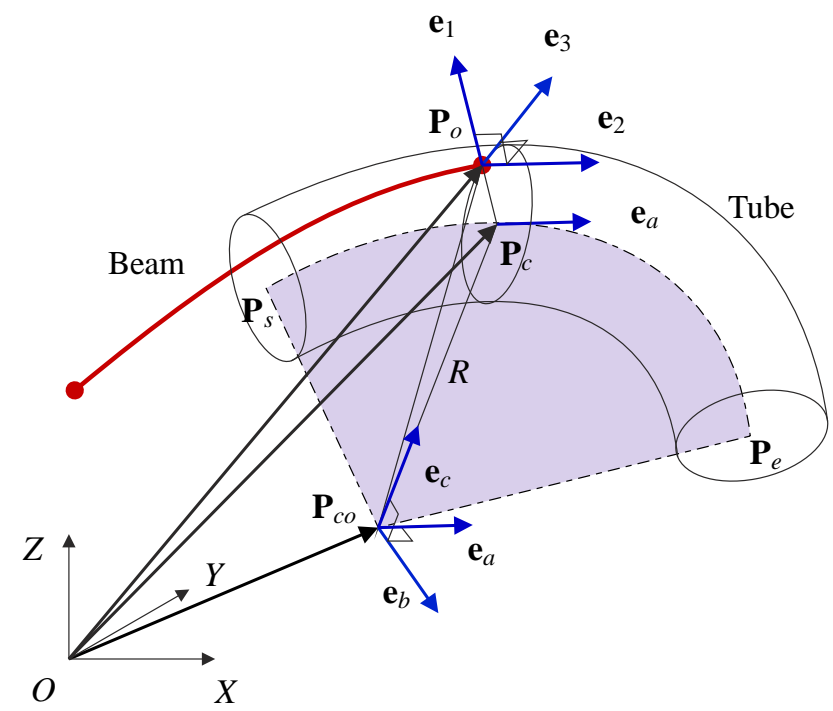

Fig. 4.4: Contact triad at the interacting node $\mathbf{P}_{o}$ of the beam when in contact with the circular tube

The base point $\mathbf{P}_{c}$ on the centerline is calculated analytically. As the crosssection of the circular tube containing the interacting node $\mathbf{P}_{o}$ is perpendicular to the plane containing the arc, the tangent vector $\mathbf{e}_{a}$ at the base point $\mathbf{P}_{c}$ is obtained from the vectors $\left(\mathbf{P}_{o}-\mathbf{P}_{c o}\right)$ and $\mathbf{e}_{b}$ as

$$
\mathbf{e}_{a}=\frac{\mathbf{e}_{b} \times\left(\mathbf{P}_{o}-\mathbf{P}_{c o}\right)}{\left\|\mathbf{e}_{b} \times\left(\mathbf{P}_{o}-\mathbf{P}_{c o}\right)\right\|} .
$$

The radial vector $\mathbf{e}_{c}$ from the center of the circular arc to the base point $\mathbf{P}_{c}$ is then calculated from the cross product of the vectors $\mathbf{e}_{a}$ and $\mathbf{e}_{b}$ as

$$
\mathbf{e}_{c}=\mathbf{e}_{a} \times \mathbf{e}_{b} .
$$

The position of the base point $\mathbf{P}_{c}$ is then given by

$$
\mathbf{P}_{c}=\mathbf{P}_{c o}+R \mathbf{e}_{c} .
$$

Figure 4.4 shows the contact triad defined at the interacting node $\mathbf{P}_{o}$ of the beam when the node is in contact with the inner wall of the circular tube.

\section{Instantaneous velocity at the point of contact}

As the instrument has a certain thickness, the actual contact point $\mathbf{P}$ lies on the outer surface of the instrument. If the interacting node has a translational 


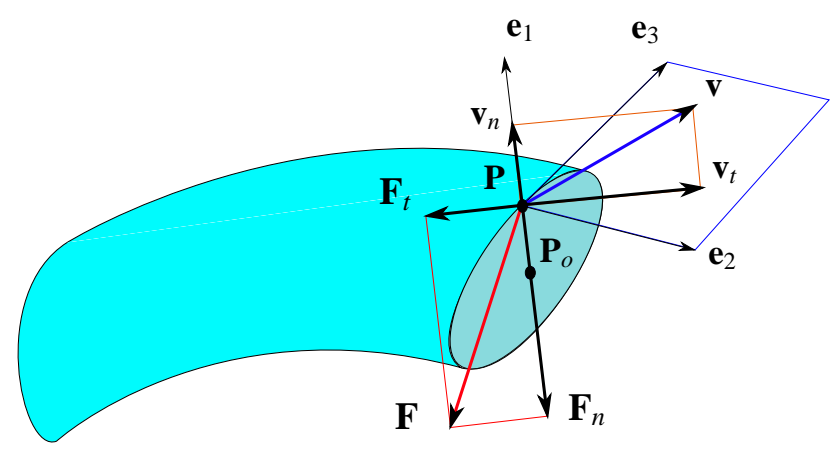

Fig. 4.5: Velocity and force components acting on the instrument at the point of contact $\mathbf{P}$

velocity $\mathbf{v}_{o}$ and angular velocity $\boldsymbol{\omega}_{o}$, the instantaneous velocity $\mathbf{v}$ at the contact point $\mathbf{P}$ is given by

$$
\mathbf{v}=\mathbf{v}_{o}+r_{o} \omega_{o} \times \mathbf{e}_{1}
$$

where $r_{o}$ is the radius of the flexible instrument.

The velocity vector $\mathbf{v}$ can be decomposed into normal and tangential components

$$
\mathbf{v}=v_{n} \mathbf{e}_{n}+v_{t} \mathbf{e}_{t}
$$

where $v_{n}=\mathbf{v} \cdot \mathbf{e}_{1}$ is the velocity component normal to the wall and the normal direction is given by $\mathbf{e}_{n}=\mathbf{e}_{1}$. The tangential velocity component $v_{t}$ is given by

$$
v_{t}=\left\|\left(\mathbf{v} \cdot \mathbf{e}_{2}\right) \mathbf{e}_{2}+\left(\mathbf{v} \cdot \mathbf{e}_{3}\right) \mathbf{e}_{3}\right\|
$$

and the tangential direction $\mathbf{e}_{t}$ is given by

$$
\mathbf{e}_{t}=\frac{\left(\mathbf{v} \cdot \mathbf{e}_{2}\right) \mathbf{e}_{2}+\left(\mathbf{v} \cdot \mathbf{e}_{3}\right) \mathbf{e}_{3}}{v_{t}}
$$

Figure 4.5 shows the velocity components at the point of contact $\mathbf{P}$.

\subsubsection{Contact Force Model}

The contact between the beam and the curved tube is defined at the nodes of the beam elements and at a point halfway between the nodes. The node experiences a normal force depending on the depth of penetration and the rate of penetration. The wall stiffness and damping are defined normal to the surface. Friction at the contact point is also defined. Therefore, depending on whether there is any sliding motion at the contact point, the node experiences a friction force in the tangential direction. 


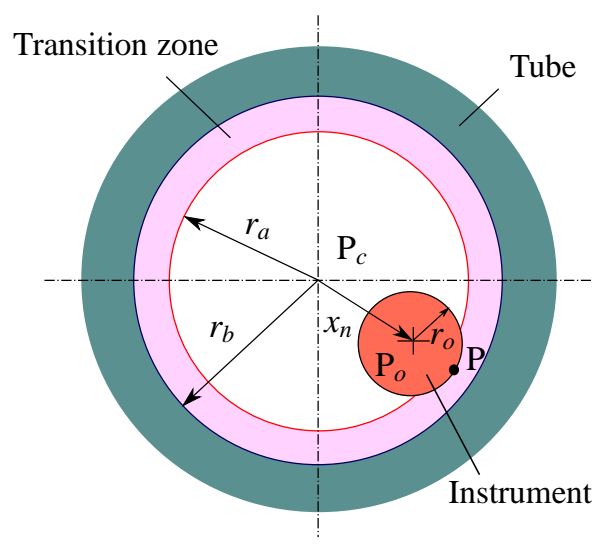

Fig. 4.6: Cross-sections of the tube and the instrument at the point of contact

Normal Force Three contact regions are defined-no contact, full contact, and transition. Figure 4.6 shows the cross-sections of the tube and the instrument at the contact. A transition zone is defined between $r_{a}$ and $r_{b}$ where the wall stiffness and damping varies continuously. The transition zone makes the normal reaction force continuous and makes the overall computation faster [26,31]. A dimensionless parameter $\xi$ is defined as $\left(x_{n}-a\right) /(b-a)$ such that $\xi$ varies from 0 to 1 as the instrument enters into the transition zone and leaves it. Here, $x_{n}$ is the normal displacement of the contact node from the centerline of the tube; $a$ and $b$ are given by $\left(r_{a}-r_{o}\right)$ and $\left(r_{b}-r_{o}\right)$ respectively.

Therefore, the net normal reaction force $F_{n}$ is given by

$$
F_{n}= \begin{cases}0 & \text { if } x_{n}<a \\ -(k / 2)(b-a) \xi^{2}-c_{w}(3-2 \xi) \xi^{2} v_{n} & \text { if } a \leq x_{n} \leq b \\ -k(b-a)(\xi-1 / 2)-c_{w} v_{n} & \text { if } x_{n}>b\end{cases}
$$

where $k$ is the wall stiffness, $c_{w}$ is the wall damping coefficient, and $v_{n}$ is the velocity in the normal direction. If the value of $F_{n}$ is positive, it is replaced by zero.

Friction Force The friction force is calculated from a Coulomb friction force model that is made a continuous function of the sliding speed $v_{t}$ at zero and is given by

$$
F_{t}=F_{c} \tanh \left(c_{v} v_{t}\right)
$$

where $F_{c}=\mu F_{n}$ is the Coulomb friction, $\mu$ is the coefficient of friction between the contacting surfaces, $F_{n}$ is the normal reaction force at the contact 
(Eqn. (4.16)), and $c_{v}$ is the velocity coefficient which determines the width of the transition region near zero sliding velocity, $v_{t}$ [26]. The friction force is directed to the opposite direction of the sliding velocity.

\section{Resultant force and moment acting at the interacting node $\mathbf{P}_{o}$}

The resultant force $\mathbf{F}$ acting at the contact point $\mathbf{P}$ of the instrument by the inner wall of the tube is given by

$$
\mathbf{F}=F_{n} \mathbf{e}_{n}+F_{t} \mathbf{e}_{t}
$$

where $F_{n}$ and $F_{t}$ are the normal and tangential forces given by Eqn. (4.16) and (4.17) respectively. Figure 4.5 shows the force components acting on the instrument at the point of contact $\mathbf{P}$.

Therefore, the resultant force $\mathbf{F}_{o}$ and moment $\mathbf{M}_{o}$ acting at the interacting node $\mathbf{P}_{o}$ due to the total interaction force $\mathbf{F}$ acting at the contact point $\mathbf{P}$ are given by

$$
\begin{aligned}
\mathbf{F}_{o} & =\mathbf{F} \\
\mathbf{M}_{o} & =r_{o} \mathbf{e}_{n} \times \mathbf{F} .
\end{aligned}
$$

The contact forces are transformed to equivalent nodal forces by equating the virtual work of the two force systems. The virtual displacement of the nominal contact point is found from the virtual displacements and rotations of the instrument. The total virtual work of the contact forces is found by integrating the virtual work of the distributed load over the length of the element. The integral is approximated by a numerical integration by a three-point scheme based on the contact forces and moments at the nodes and at the halfway point. For the detailed description, the readers can refer to [42].

\subsection{Simulation}

The interaction force between the instrument and the curved tube is implemented in the SPACAR program through a user-defined routine. The axial deformation of the beam element is suppressed to limit the highest natural frequency in the system and, thus, reducing the computation time.

The developed 3-D flexible multibody model is used for the simulation of fine manipulation in translation and rotation under different loading conditions. We studied the motion and force transmission of the instrument. The details of the various simulations are presented in the following sections. 


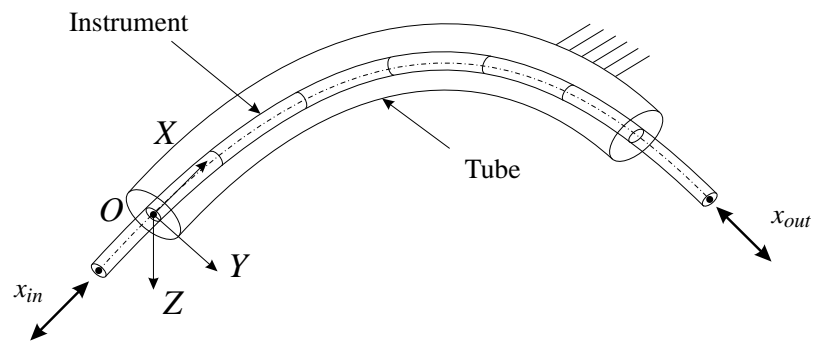

Fig. 4.7: Configuration of the planar tube with the instrument

\subsubsection{Fine Manipulation in Translation in a Curved Rigid Tube}

In order to understand the transmission characteristics of the instrument inside an endoscope in the presence of friction, a curved rigid tube is defined by a circular section of 90 degrees of radius $300 \mathrm{~mm}$. The tube is defined in the $x y$-plane. Figure 4.7 shows the configuration of the curved tube with the instrument. Two straight tubes are defined at the entry and exit of the curved tube to further guide the instrument ends. The instrument is defined by a stainless steel wire of $0.5 \mathrm{~mm}$ diameter. The length of the instrument inside the curved section is defined by 10 equal length spatial beam elements. Two beam elements of $50 \mathrm{~mm}$ length are defined at the ends. An input motion along the $x$-direction $x_{i n}$ is prescribed at the proximal end. The output motion of the distal end along the longitudinal axis $x_{\text {out }}$ is observed. A small stroke sine input motion $x_{i n}$ is applied as follows

$$
x_{i n}(t)=A \sin (\omega t)
$$

where $A$ is the amplitude and $\omega=2 \pi f_{n}$. Here, the amplitude $A$ is $20 \mathrm{~mm}$ and the frequency $f_{n}$ is $1 \mathrm{~Hz}$. The velocity $\dot{x}_{i n}$ and the acceleration $\ddot{x}_{i n}$ are also prescribed at the input node accordingly. In this case, the output motion $x_{\text {out }}$ is along the $y$-direction.

\section{No load at the distal end}

At first, the simulations are performed in which there is no load attached to the distal end. The instrument is assumed straight in the beginning. The initial deformations of the elements are obtained analytically and prescribed in the beginning to place the instrument along the centerline of the curved rigid tube. The instrument remains constrained inside the tube during the simulation due to the interaction with the tube wall. The data used for the model and simulation are given in Table 4.1. 
Table 4.1: Data used for the simulations

\begin{tabular}{llc}
\hline Description & Values & Unit \\
\hline Density, $\rho$ & 7.8 & $10^{3} \mathrm{~kg} / \mathrm{m}^{3}$ \\
Young's modulus, $E$ & 200 & $10^{9} \mathrm{~N} / \mathrm{m}^{2}$ \\
Shear modulus, $G$ & 79 & $10^{9} \mathrm{~N} / \mathrm{m}^{2}$ \\
Radius of the wire, $r_{o}$ & 0.25 & $\mathrm{~mm}$ \\
Mass/length, $\rho A$ & 1.532 & $10^{-3} \mathrm{~kg} / \mathrm{m}$ \\
Flexural rigidity, $E I$ & 6.14 & $10^{-4} \mathrm{~N} \mathrm{~m}^{2}$ \\
Torsional rigidity, $G J$ & 4.85 & $10^{-4} \mathrm{~N} \mathrm{~m}^{2}$ \\
\hline Inner radius of the tube, $r_{a}$ & 2.0 & $\mathrm{~mm}$ \\
Size of the transition zone & 0.5 & $\mathrm{~mm}$ \\
Wall stiffness, $k$ & 2.0 & $10^{4} \mathrm{~N} / \mathrm{m}^{2}$ \\
Wall damping, $c_{w}$ & 10.0 & $\mathrm{Ns} / \mathrm{m}^{2}$ \\
Velocity coefficient, $c_{v}$ & 1.0 & $10^{3}(\mathrm{~m} / \mathrm{s})^{-1}$ \\
\hline
\end{tabular}

Effect of the coefficient of friction $\mu$. Various simulations are performed for different values of the coefficient of friction $\mu$ between the instrument and the curved tube. The motion of the distal end $x_{\text {out }}$ is compared with the motion of the proximal end $x_{i n}$. The simulation results are shown in Fig. 4.8. As the value of $\mu$ increases, the motion hysteresis also increases. During the retraction of the instrument, the distal end follows the proximal end closely. However, as the direction of motion changes and the instrument starts moving forward inside the curved tube, the distal end does not follow the input motion instantly. Moreover, the motion of the distal end is oscillatory during this stroke. This is more evident when we compare the velocity of the ends.

Figure 4.9 shows the velocity of the distal end $v_{\text {out }}$ for different values of $\mu$. The velocity of the proximal end $v_{i n}$ is also shown for the comparison. The stick-slip behavior is clearly evident at the end of the stroke when the velocity changes sign. Moreover, the motion is smoother in the retraction stroke while it is oscillatory in the other stroke.

\section{Axial load at the distal end}

A load is applied at the distal end along the $y$-direction via an extension spring of stiffness $\left(k_{s p}=120 \mathrm{~N} / \mathrm{m}\right)$. An initial extension of $60 \mathrm{~mm}$ is defined so that there is always a tensile force acting on the instrument during the entire motion. 


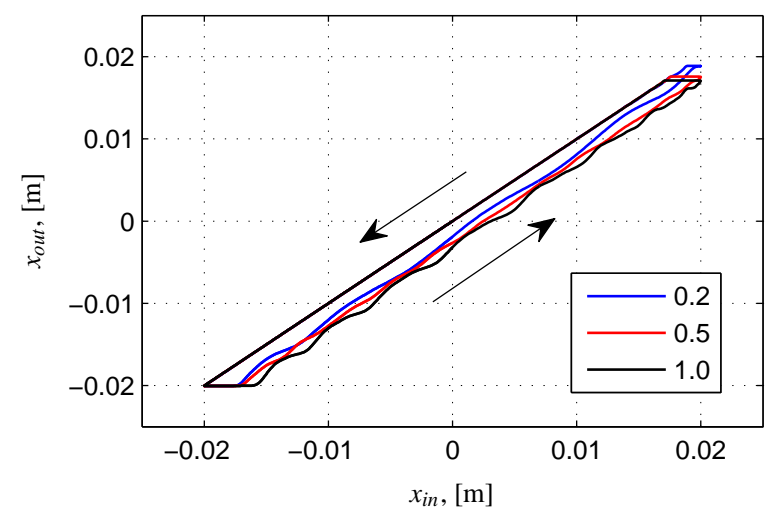

Fig. 4.8: Motion hysteresis for different values of $\mu$

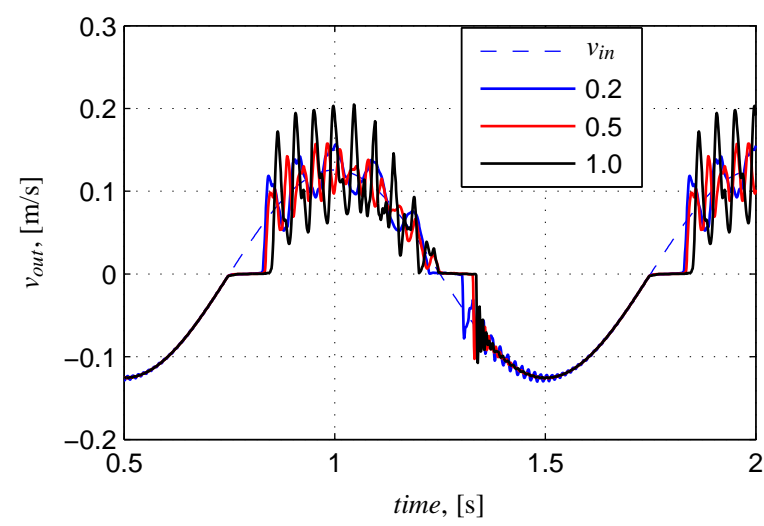

Fig. 4.9: The velocity of the distal end $v_{\text {out }}$ is compared for different values of $\mu$

The output motion $x_{\text {out }}$ is compared with the input motion $x_{i n}$. The motion characteristic of the instrument is compared with the no-load case. The value of $\mu$ used for the simulations is 0.2 . Figure 4.10 shows the motion hysteresis with and without axial load. In the preloaded case, the backlash is reduced to $0.6 \mathrm{~mm}$ from $2.8 \mathrm{~mm}$ in the no-load case. The preloading in one direction helps in reducing the motion hysteresis.

Figure 4.11 shows the velocity of the distal end $v_{\text {out }}$ with and without the axial load. The distal end is closely following the input motion in the case of the preloaded case. The motion is without oscillations in both the strokes, unlike the no-load case. The instrument shows the stick-slip behavior near the zero speed motion. 


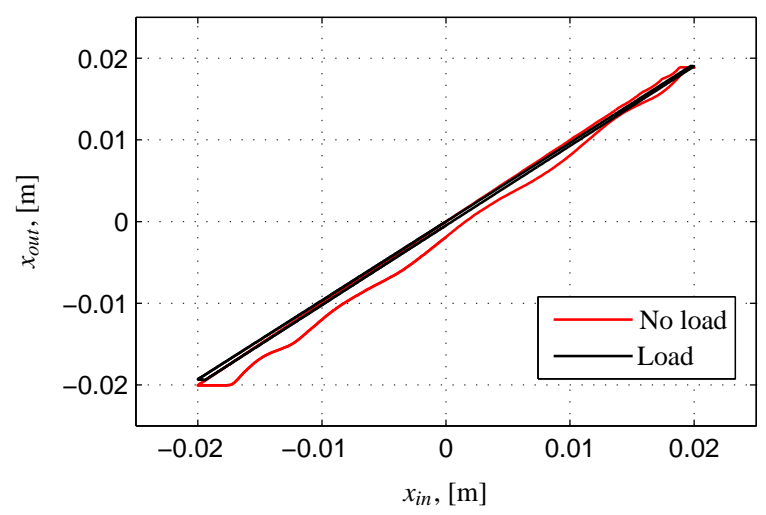

Fig. 4.10: Motion hysteresis with and without axial load for $(\mu=0.2)$

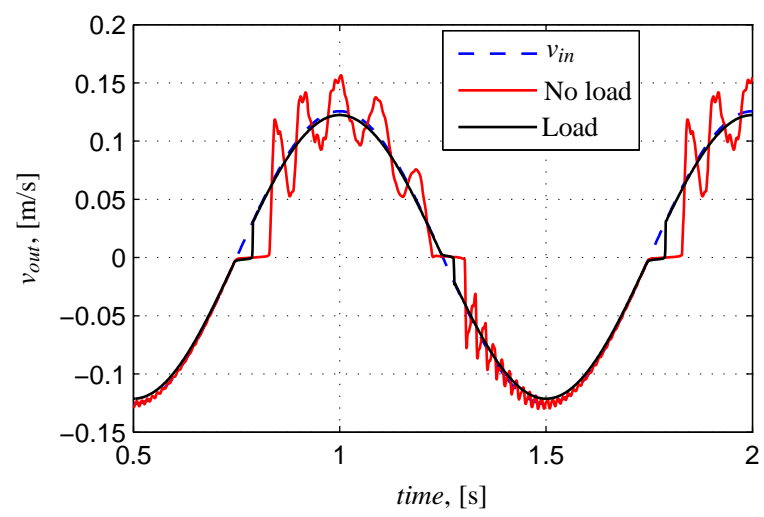

Fig. 4.11: The velocity of the distal end $v_{\text {out }}$ with and without axial load for $(\mu=0.2)$

Force transmission. The force transmission from the proximal end to the distal end is also analyzed. The forces at the proximal end $F_{\text {in }}$ is compared with the force at the distal end $F_{\text {out }}$ (see Fig. 4.12). Here, $F_{\text {out }}$ is equal to the spring force $F_{s p}$. During the retraction of the instrument, the force at the proximal end has to overcome the spring force and also the friction force; and therefore $F_{\text {in }}$ is larger than $F_{\text {out }}$. However, as the direction of motion changes at the end of the stroke and the instrument starts moving forward, the friction force acts opposite to the spring force; and therefore $F_{\text {in }}$ is smaller than $F_{\text {out }}$.

The force transmission in the case of the axial loading can be compared with the capstan equation. The detailed analysis is presented in the following section. 


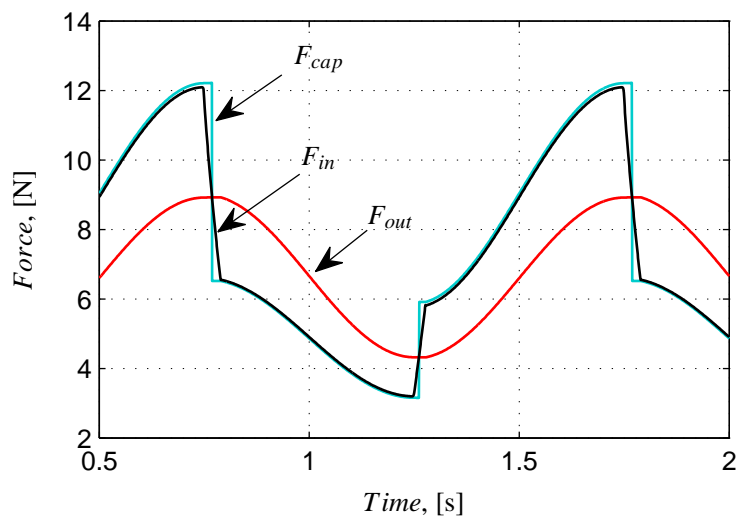

Fig. 4.12: Comparison of the forces at the proximal end $F_{\text {in }}$ and at the distal end $F_{\text {out }}$. The force $F_{\text {cap }}$ based on the capstan equation is also shown.

\section{Comparison with the capstan equation}

The well-known relationship between the output and input tensions on the both sides of a flexible string wound around a circular shaped body is given by the capstan equation. In the axially preloaded case, the capstan equation can be written as

$$
\frac{F_{\text {in }}}{F_{\text {out }}}=e^{ \pm \mu \theta}
$$

Here, the sign is positive when the instrument is retracting and it is negative when the instrument is moving forward. The input force based on Eqn. (4.21), $F_{c a p}$, is also shown in Fig. 4.12. The input force $F_{\text {in }}$ obtained from the simulation result is closely following the input force based on the capstan equation $F_{c a p}$. However, near the zero velocity region, the input force $F_{\text {in }}$ is not changing abruptly as compared to the estimated force $F_{c a p}$. Note that we used a continuously varying Coulomb friction force model near zero sliding speed which is dependent on the velocity coefficient $c_{v}$ (see Eqn. (4.17)). The slope in $F_{\text {in }}$ near zero sliding speed is related to the stiffness of the spring.

Eqn. (4.21) can be further written as

$$
\ln \left(\frac{F_{\text {in }}}{F_{\text {out }}}\right)= \pm \mu \theta \text {. }
$$

As it is evident from Eqn. (4.22), the logarithm of the force ratio is constant and jumps from $\mu \theta$ to $-\mu \theta$ as the direction of motion changes. Figure 4.13 shows the plot of the logarithm of the force ratio with respect to the output force $F_{\text {out }}$. The simulation result is compared with the analytical result. The 


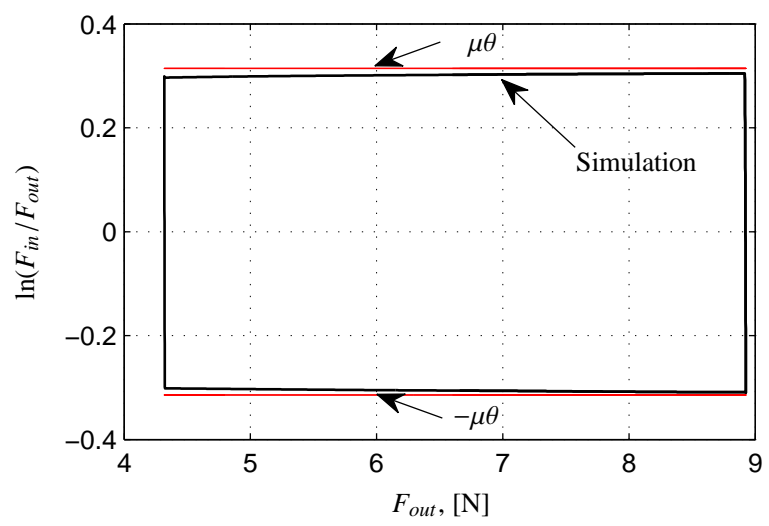

Fig. 4.13: Comparison of the logarithm of the force ratio at the ends, $\ln \left(F_{\text {in }} / F_{\text {out }}\right)$, with the capstan equation $(\mu=0.2, \theta=\pi / 2)$

plot will be used later for the estimation of the value of $\mu$. The simulation result shows a deviation from the capstan equation. The percentage deviation of the logarithm of the force ratio is calculated with respect to the value of $\mu \theta$ for the both strokes of motion. The effect of the bending rigidity of the instrument and the magnitude of the axial load applied at the distal end on the percentage deviation is also studied. The results are presented in Table 4.2.

Effect of the bending rigidity. The capstan equation does not take the bending rigidity of the wire into consideration. However, several researchers have either included the bending rigidity in the formulation and modified the capstan equation or analyzed the effect of the bending rigidity $[23,56]$. For the validation of the proposed model, the bending rigidity $E I$ of the instrument is reduced and the percentage deviation is compared. The results are shown in Table 4.2. We observed that the percentage deviation only reduced marginally in this case. Further reduction in the bending rigidity does not reduce the deviation.

Effect of the axial load. The magnitude of the axial load acting on the distal end can reduce the effect of the bending rigidity on the force transmission. The larger the magnitude of the axial load, the less will be the effect of bending rigidity on the force transmission. The amount of the axial load is reduced by decreasing the spring stiffness attached at the distal end. The percentage deviation from the capstan equation is shown in Table 4.2. We observed a marginal change in the percentage deviation. The deviation does not increase with the 
Table 4.2: Percentage deviation from the capstan equation

\begin{tabular}{|c|c|c|}
\hline \multicolumn{3}{|c|}{$\begin{array}{ll} & \text { Retraction Push-in } \\
\end{array}$} \\
\hline \\
\hline$E I$ & $3.1-5.6$ & $1.7-4.0$ \\
\hline$\left(1.0 \times 10^{-2}\right) E I$ & $3.0-5.1$ & $1.6-3.8$ \\
\hline$\left(1.0 \times 10^{-6}\right) E I$ & $3.0-5.1$ & $1.6-3.8$ \\
\hline \multicolumn{3}{|c|}{ Effect of axial load } \\
\hline$\overline{k_{s p}}$ & $3.1-5.6$ & $1.7-4.0$ \\
\hline$\left(1.0 \times 10^{-1}\right) k_{s p}$ & $2.4-5.3$ & $1.2-2.9$ \\
\hline
\end{tabular}

reduction in the axial load as expected.

The maximum percentage deviation observed in this case is $5.6 \%$. This corresponds to a deviation of $1.8 \%$ in the estimation of $F_{i n}$. In the present configuration, the radius of curvature of the rigid curved tube over which the wire is bent is 1200 times the radius of the wire. The effect of the bending rigidity of the instrument and the magnitude of the axial load may be significant if the radius of the curvature of the curved tube is small. However, the simulation results already show good agreement with the capstan equation. The effect of number of elements were also performed, but we did not observe a significant change. The other parameter which can affect the transmission behavior is the wall stiffness. The value of the wall stiffness is chosen to reduce the overall computational time.

\subsubsection{Fine Manipulation in Rotation in a Curved Rigid Tube}

In order to understand the motion and torque transmission of the instrument inside the curved rigid tube, we performed the simulation for the fine manipulation in rotation. The configuration of the model is same as for the fine manipulation in translation as shown in Fig. 4.7. A rotation motion input is given to the proximal end through a hinge element.

\section{No load at the distal end}

There is no load applied at the distal end. The instrument is straight in the beginning and an initial deformation is applied to the individual beam elements so that the instrument takes the shape of the curved rigid tube. Various simulations are performed to study the effect of friction on the motion and torque characteristics in rotation. A sinusoidal motion input in rotation $\theta_{i n}$ is given to 


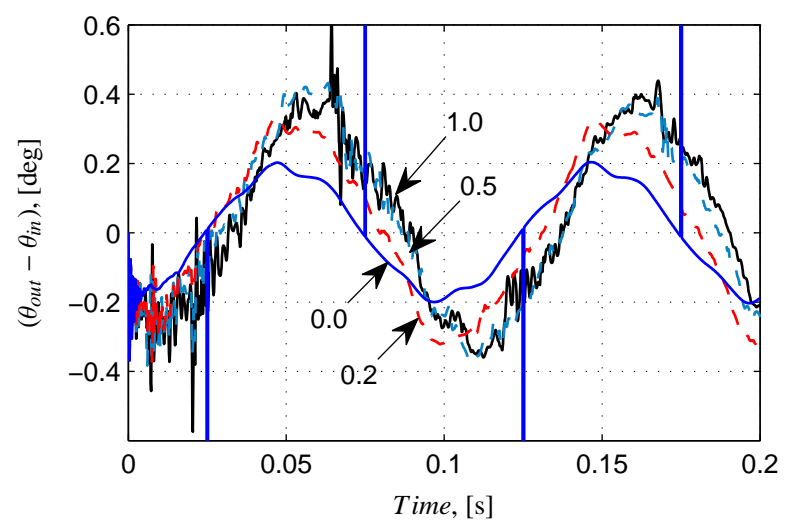

Fig. 4.14: Difference in angular displacement for different values of $(\mu=0.0,0.2,0.5,1.0)$

the proximal end as follows:

$$
\theta_{\text {in }}=A \sin (\omega t)
$$

where $A=\pi / 2$ is the amplitude of the rotation input and $\omega=2 \pi f_{n}$. Here, the frequency $f_{n}$ is $10 \mathrm{~Hz}$. The angular velocity $\dot{\theta}_{\text {in }}$ and the acceleration $\ddot{\theta}_{i n}$ are also prescribed. A higher value of $f_{n}$ is used as the simulation is faster. A smaller value of $f_{n}$ increases the computation time considerably.

Effect of the coefficient of friction $\mu$. The rotation of the distal end $\theta_{\text {out }}$ is observed for different values of $(\mu=0.0,0.2,0.5,1.0)$. Figure 4.14 shows the plot of the difference in angular displacement $\left(\theta_{\text {out }}-\theta_{\text {in }}\right)$ for different values of $\mu$. For the frictionless contact $(\mu=0.0)$, the difference in angular displacement is of the order of \pm 0.2 degree. The difference is due to the inertia as the angular velocity is not constant. As the value of $\mu$ increases, the difference in angular displacement also increases. For $\mu=1.0$, the difference is of the order of \pm 0.4 degree. Due to the additional friction torque acting at the various nodes, the difference in the angular displacement will increase. Note that the plots cover the first two periods only and the transient effects are clearly visible.

The moments at the proximal end are obtained from the simulation results for different values of $\mu$. The moment along the longitudinal axis $M_{x}$ is equal to the driving torque. In this case, it will be equal to the inertia torque and the friction torque. Figure 4.15 shows the plot of the moment along $x$-axis at the proximal end for different values of $\mu$. For the frictionless case, the moment $M_{x}$ is equal to the inertia torque only. However, as the value of $\mu$ increases, the moment $M_{x}$ has to overcome the increased friction torque also. 


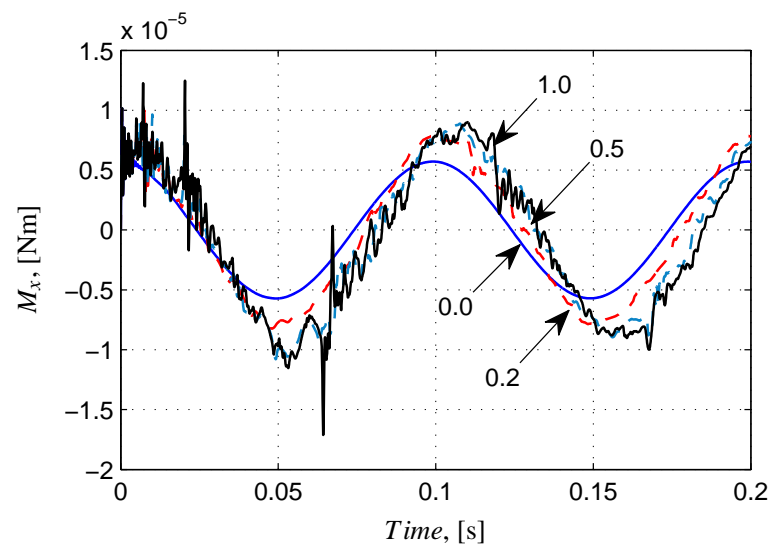

Fig. 4.15: Moment at the proximal end along the $x$-axis $M_{x}$ for different values of $(\mu=$ $0.0,0.2,0.5,1.0)$

The flexible instruments often exhibit whipping motion in rotation $[42,60]$. Different flexural rigidity along the two principal axes of the instrument and the pre-curvature can affect the motion transmission and can render the instrument unusable and dangerous. Therefore, it is important that the developed model should be able to predict the motion characteristics.

Effect of different flexural rigidity along the two principal axes $\left(E I_{y} \neq\right.$ $\left.E I_{z}\right)$. The parameter $p$ defines the deviation from the mean value of the flexural rigidity $E I$ such that $E I_{y}=(1+p) E I$ and $E I_{z}=(1-p) E I$. The motion and force characteristics are studied for different values of $p$. The value of $\mu$ used for the simulations is 0.2. There is no load applied at the distal end. Again, the sinusoidal motion input in rotation is prescribed at the proximal end.

The lag in angular displacement $\left(\theta_{\text {in }}-\theta_{\text {out }}\right)$ is obtained from the simulation results for the various values of $p$. Figure 4.16 shows the plots of the lag in angular displacement with respect to the input rotation $\theta_{i n}$. For the smaller values of $p$, the lag is gradual. As the value of $p$ increases, the amount of lag also increases. At a larger value of ( $p=0.3)$, the whipping phenomenon is observed. The distal end is almost stationary and then suddenly unwinds. The transient vibration can be observed. The moment along the $x$-axis $M_{x}$ also shows the same effect.

Effect of pre-curvature. The effect of pre-curvature is studied using the developed model. An initial curvature with a radius of $\left(R_{c_{1}}=0.3 \mathrm{~m}, R_{c_{2}}=\right.$ $1.2 \mathrm{~m}$ ) is prescribed to the wire. A constant angular rotation of 2 revolutions 


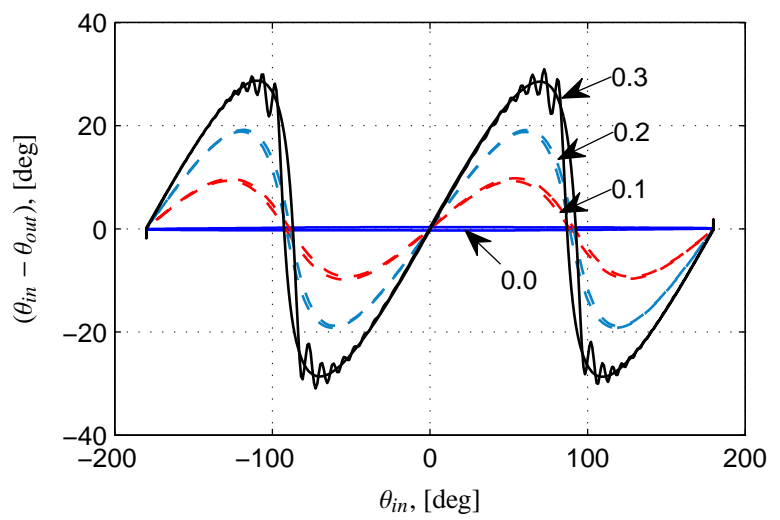

Fig. 4.16: Lag in angular displacement $\left(\theta_{\text {in }}-\theta_{\text {out }}\right)$ for different values of $(p=0.0,0.1,0.2,0.3)$

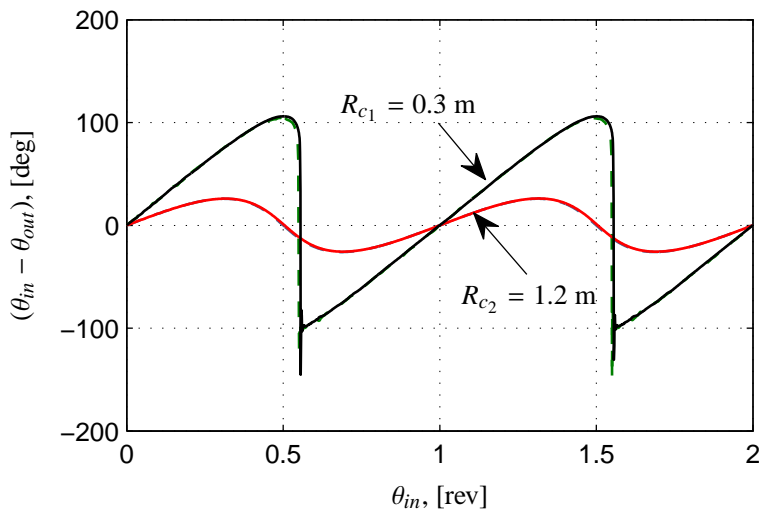

Fig. 4.17: Lag in the angular displacement, $\left(\theta_{\text {in }}-\theta_{\text {out }}\right)$, for different values of pre-curvature with a radius of $\left(R_{c_{1}}=0.3 \mathrm{~m}, R_{c_{2}}=1.2 \mathrm{~m}\right)$ and $\mu,(--)$ for $\mu=0.0$, and (一) for $\mu=0.5$

per second is given to the proximal end. There is no load applied at the distal end.

The lag in the angular displacement, $\left(\theta_{\text {in }}-\theta_{\text {out }}\right)$, is calculated for different values of pre-curvature and $\mu$. Figure 4.17 shows the plot of the lag in the angular displacement for $(\mu=0.0,0.5)$. The whipping phenomenon is clearly observed in the case of pre-curvature with smaller radius $\left(R_{c_{1}}=0.3 \mathrm{~m}\right)$. The amount of lag in the angular displacement is continuously increasing to the maximum and suddenly unwinds and gives rise to the whipping action. However, in the case of pre-curvature with larger radius $\left(R_{c_{2}}=1.2 \mathrm{~m}\right)$, the whipping action does not take place. The friction has a negligible effect. 


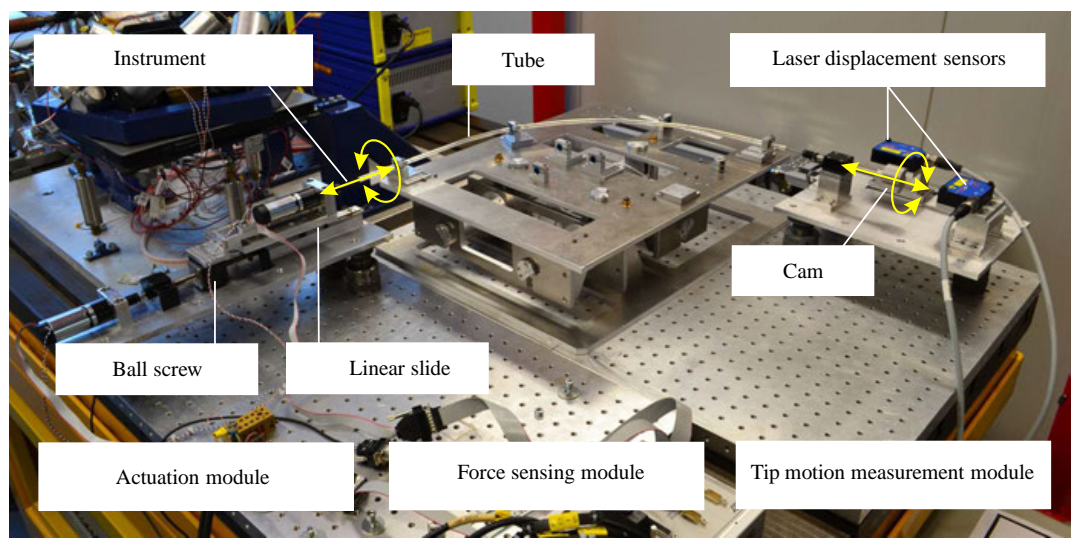

Fig. 4.18: Experimental set-up showing the key modules

\subsubsection{Discussion}

The developed flexible multibody model incorporates all the required/necessary parameters to study the dynamic behavior of the instrument in translation and rotation inside a curved rigid tube. We performed various simulations using the developed flexible multibody model. The simulations in translation and rotation showcases the typical behavior of the wire under different scenarios. The simulation results conform to the dynamic behavior reported in the literature. However, experimental validation is required for the confidence in using the model. The experimental set-up and the experimental validation is explained in detail in the following section.

\subsection{Experimental Set-up and Validation}

An experimental set-up was designed to validate the model and simulation results. The set-up consists of three basic modules: Actuation module (AM), Force sensing module (FSM), and Tip motion measurement module (T3M). Figure 4.18 shows the whole assembly of the experimental set-up, indicating the three modules together with the key components.

The AM provides actuation in 2-DOFs, i.e. translation and rotation along the longitudinal axis of the instrument at the proximal end. The FSM enables measurement of all forces and torques arising from the interaction of the instrument with the curved rigid tube. The T3M measures the translation and rotation of the distal end of the instrument along the longitudinal axis. The translation and rotation of the distal end is measured via a specially designed 


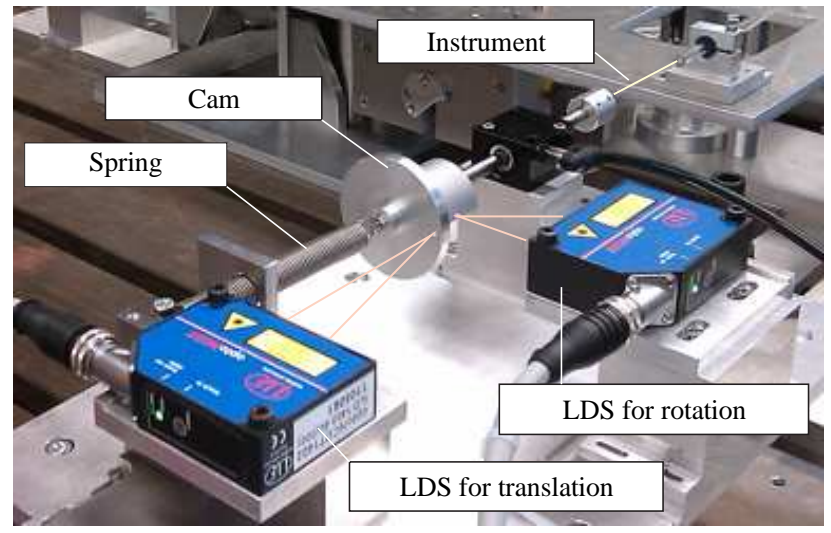

Fig. 4.19: Experimental set-up with the extension spring attached to the distal end of the wire

cam through two laser displacement sensors (LDS). The details of the design and evaluation of the set-up can be referred to [27,30].

The set-up is configured as shown in Fig. 4.18. A glass tube is bent to a circular arc of 90 degrees and of radius $300 \mathrm{~mm}$. A stainless steel wire of diameter $0.5 \mathrm{~mm}$ and length $800 \mathrm{~mm}$ is chosen for the model validation. The glass tube is chosen so that the wire can be observed from outside. The curved glass tube is rigidly attached to the top floating plate of the FSM. The straight sections of the tube at the entry and exit are aligned along the $x$ - and $y$-directions. The ends of the wire are attached to the AM and the T3M or the load as per the requirement.

Various experiments are performed to validate the developed model for motion and force transmission in translation and rotation.

\subsubsection{Motion and Force Transmission During Translation}

An extension spring is attached to the distal end of the wire to preload the wire in tension as shown in Fig. 4.19. The proximal end of the wire is attached to the AM. An initial extension is prescribed so that the wire is always in tension during the entire motion. A sinusoidal motion is given to the proximal end.

Figure 4.20 shows the plot of the forces and moments in the $x y z$-directions measured by the FSM. The input force $F_{\text {in }}$ acting on the proximal end of the wire is equal and opposite to the reaction force in $x$-direction $F_{x}$ measured by the FSM. The output force $F_{\text {out }}$ acting at the distal end of the wire is equal and opposite to the reaction force in $y$-direction $F_{y}$ measured by the FSM. Moreover, the output force $F_{\text {out }}$ is equal to the spring force. The measured signal is filtered off-line, forward and backward in time to achieve zero-phase 

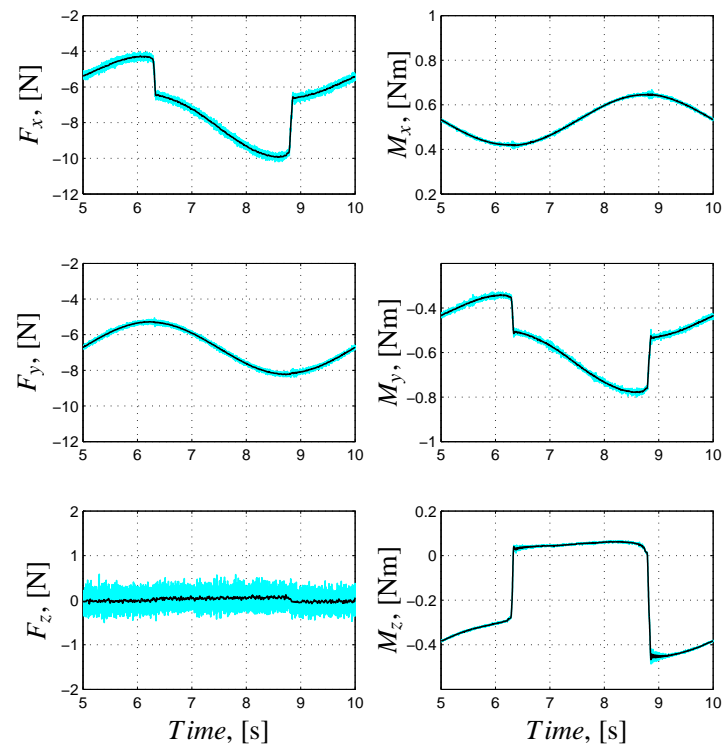

Fig. 4.20: Forces and moments in $x y z$-directions measured by the FSM

digital filtering. A 2nd order low-pass digital Butterworth filter is designed with cut-off frequency of $45 \mathrm{~Hz}$ [30].

The output force $F_{\text {out }}$ depends on the extension of the spring attached to the distal end. As the proximal end moves, the distal end follows the motion; and accordingly we observe a gradual change in the output force $F_{\text {out }}$. The input force $F_{\text {in }}$ has to overcome the friction force and the spring force during retraction. However, at the end of the retraction stroke as the direction of motion changes, the direction of the friction force also changes and the input force $F_{i n}$ has to overcome the spring force minus the friction force. The sudden change in $F_{\text {in }}$ can be observed at the end of the stroke.

The forces $F_{\text {in }}$ and $F_{\text {out }}$ also apply moments along $y$ - and $x$-axes respectively. The changes in $F_{\text {in }}$ and $F_{\text {out }}$ are reflected in the moments $M_{y}$ and $M_{x}$ respectively (see Fig. 4.20). The corresponding moments follow the same pattern as expected.

Figure 4.21 shows the plot of the input and output forces $F_{\text {in }}$ and $F_{\text {out }}$ with respect to the input displacement $x_{i n}$. Both the input and the output forces $F_{\text {in }}$ and $F_{\text {out }}$ show a linear relationship during both the strokes of motion. The relationship between $F_{\text {in }}$ and $F_{\text {out }}$ will be used to estimate the coefficient of friction $\mu$ between the glass tube and the stainless steel wire, which is described in detail in the following section. 


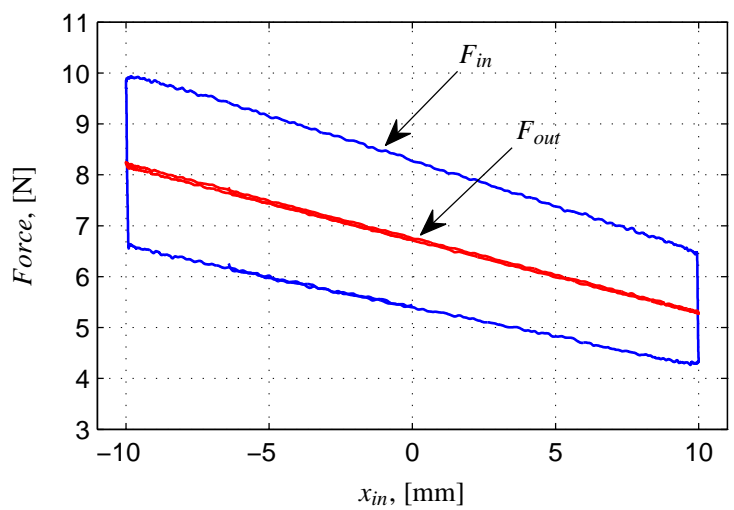

Fig. 4.21: Comparison of the input force $F_{\text {in }}$ and the output force $F_{\text {out }}$ with respect to $x_{\text {in }}$

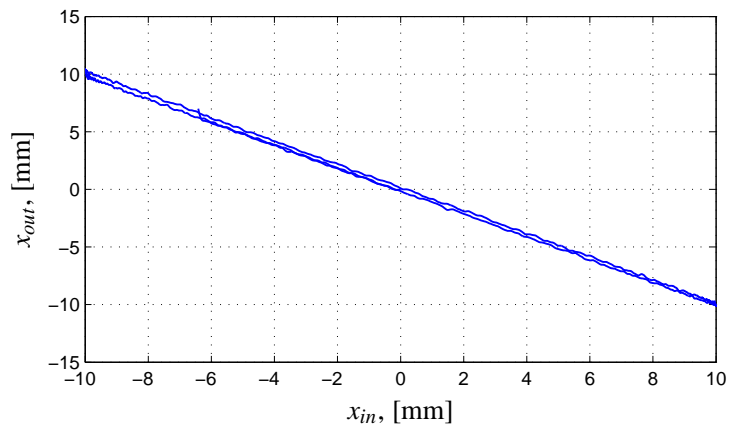

Fig. 4.22: Motion hysteresis in translation

The output motion of the distal end $x_{\text {out }}$ is calculated from the output force $F_{\text {out }}$. Figure 4.22 shows the plot of the output displacement $x_{\text {out }}$ with respect to the input displacement $x_{i n}$. A hysteresis of the order of $0.4 \mathrm{~mm}$ is observed in this case.

\section{Estimation of $\mu$ and comparison with the capstan equation}

For the estimation of the value of coefficient of friction between the contacting surfaces, the logarithm of the force ratio, $\ln \left(F_{\text {in }} / F_{\text {out }}\right)$, is calculated and compared with Eqn. (4.22). Figure 4.23 shows the plot of the logarithm of the force ratio with respect to the input displacement. The logarithm of the measured force ratio is shown by $F_{l r}$. The ratio is constant and only changing when the direction of motion changes, as expected from Eqn. (4.22). However, if we see the plot of the logarithm of the force ratio, the measured quantity is 


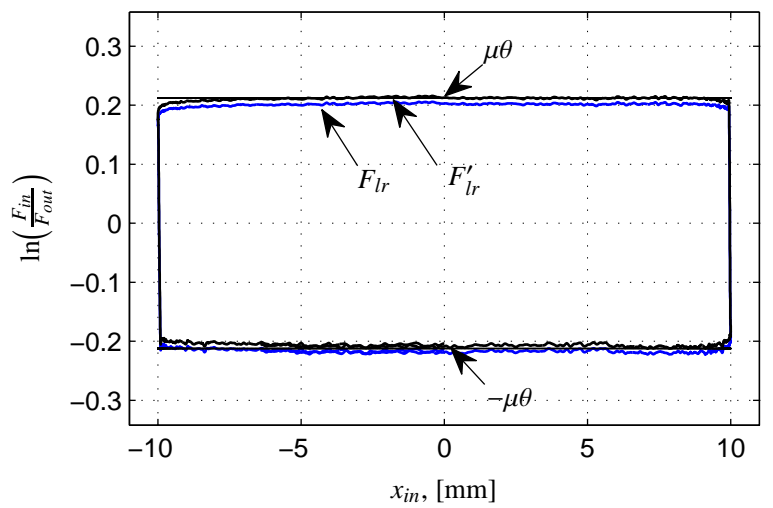

Fig. 4.23: The logarithm of the ratio of the input force and output force, $\ln \left(F_{\text {in }} / F_{\text {out }}\right)$, with respect to the input displacement

Table 4.3: Data used for the validation

\begin{tabular}{lcc}
\hline Parameter & Value Unit \\
\hline Spring stiffness, $k_{s p}$ & $144.9 \mathrm{~N} / \mathrm{m}$ \\
Initial extension & $46.5 \mathrm{~mm}$ \\
\hline
\end{tabular}

not evenly placed around zero as expected from Eqn. (4.22).

If we define a deviation from the capstan equation by a bias $\delta$, Eqn. (4.22) can be written as

$$
\ln \left(\frac{F_{\text {in }}}{F_{\text {out }}}\right)= \pm \mu \theta+\delta
$$

The bias $\delta$ is calculated from the mean value of the logarithm of the force ratio, $\ln \left(F_{\text {in }} / F_{\text {out }}\right)$, over complete cycles. The bias measured in this case is $(\delta=-0.01)$. The logarithm of the force ratio is accordingly readjusted and the modified value of the logarithm of the force ratio $F_{l r}^{\prime}$ is shown in Fig. 4.23. The bias $\delta$ corresponds to an error of $1.0 \%$ in the force measurement in this case.

The modified value of the logarithm of the force ratio is used to estimate the value of the coefficient of friction between the glass tube and the stainless steel wire: $\mu=0.135$. The value of the logarithm of the force ratio based on the capstan equation is also shown in Fig. 4.23 for the estimated value of $\mu=0.135$ and $\theta=\pi / 2$. The experimental results show a deviation of $1.0 \%$ from the capstan equation. 


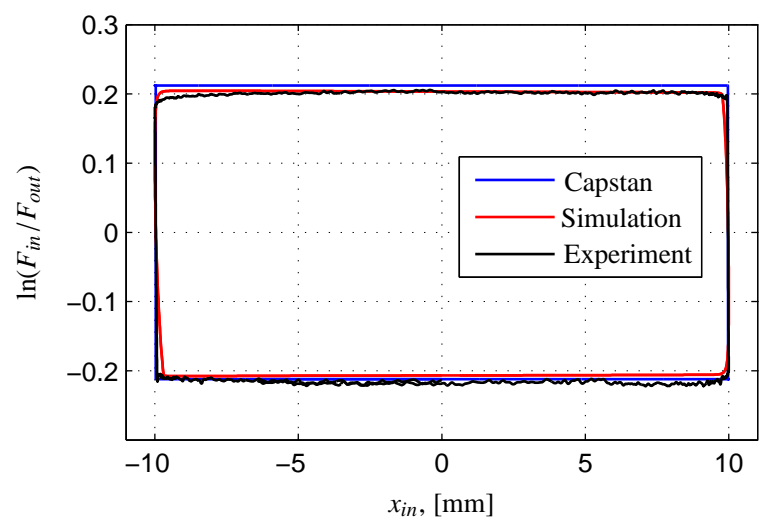

Fig. 4.24: Comparison of the logarithm of the force ratio, $\ln \left(F_{\text {in }} / F_{\text {out }}\right)$, with the simulation result

\section{Comparison with the simulation result}

A simulation is carried out using the developed flexible multibody model to compare with the experimental results. A sinusoidal input motion is prescribed in translation at the proximal end. An amplitude of $10 \mathrm{~mm}$ with a frequency of $0.2 \mathrm{~Hz}$ is used in the simulation. Here, the estimated value of the coefficient of friction $\mu$ of 0.135 is used for the contacting surfaces. An extension spring is attached at the distal end. The spring stiffness and the initial extension are stated in Table 4.3.

Figure 4.24 shows the plot of the logarithm of the force ratio, $\ln \left(F_{\text {in }} / F_{\text {out }}\right)$, with respect to the input displacement $x_{i n}$. The experimental result is compared with the simulation result. The corresponding plot based on the capstan equation is also shown. The simulation result shows a good agreement with the experimental result.

\subsubsection{Motion Transmission During Rotation}

The rotation experiment is performed by giving a sinusoidal rotation of amplitude 360 degrees at $0.5 \mathrm{~Hz}$ at the proximal end of the wire. The distal end is connected to the T3M. There is no axial load applied at the end. The output rotation of the distal end is measured with the laser displacement sensor via the designed cam. Figure 4.25 shows the plot of the rotation output of the distal end $\theta_{\text {out }}$ with respect to the rotation input of the proximal end $\theta_{i n}$. Here, an overshoot of the order of 100 degrees is observed at the end of the each stroke. This is expected as the cam and the guiding shaft have significant rotational inertia. 


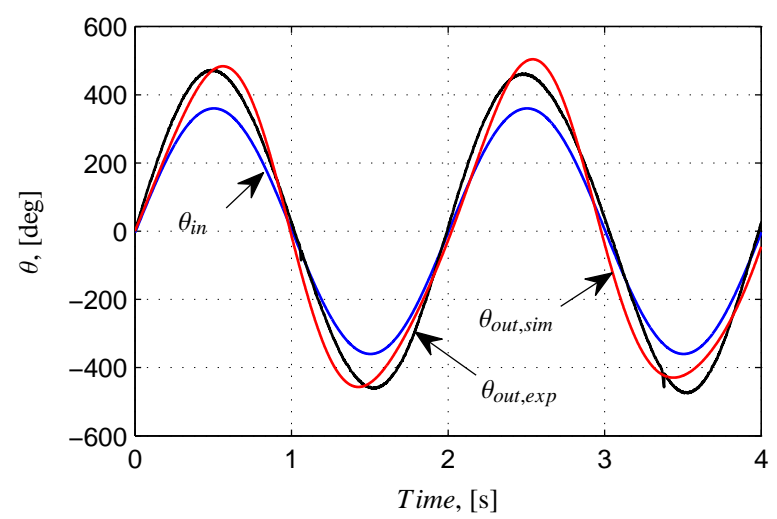

Fig. 4.25: Comparison of the rotation of the distal end $\theta_{\text {out exp }}$ with the simulation result $\theta_{\text {out,sim }}$ for the given input rotation at the proximal end $\theta_{i n}$

Table 4.4: Mass properties of the guide shaft and cam

\begin{tabular}{|c|c|c|}
\hline Parameter & Unit & Value \\
\hline Rotational inertia & $J_{x} \mathrm{~kg} \mathrm{~m}^{2}$ & $1.36 \times 10^{-5}$ \\
\hline & $J_{y} \mathrm{~kg} \mathrm{~m}^{2}$ & $17.47 \times 10^{-5}$ \\
\hline & $J_{z} \mathrm{~kg} \mathrm{~m}^{2}$ & $17.67 \times 10^{-5}$ \\
\hline Mass & $m \quad \mathrm{~kg}$ & 0.100 \\
\hline
\end{tabular}

The rotational inertia of the cam and the guiding shaft are obtained from the CAD model. The mass properties obtained from the CAD model are stated in Table 4.4. The rotational inertia along the longitudinal axis is also obtained from the fundamental frequency of the wire and the added inertia at the distal end. The fundamental frequency is $1.06 \mathrm{~Hz}$. The total length of the stainless steel wire is $800 \mathrm{~mm}$. The rotational inertia $J_{x}$ thus calculated is $1.38 \times 10^{-5} \mathrm{~kg} \mathrm{~m}^{2}$.

\section{Comparison with the simulation result}

The mass and the rotational inertia of the guide shaft and the cam are added in the model as a lumped mass and inertia at the distal end of the model. The same input rotation is prescribed at the proximal end of the model and the simulation is carried out. The output rotation of the distal end $\theta_{\text {out }}$ is calculated and compared with the experimental result as shown in Fig. 4.25.

Figure 4.26 shows the plot of the output rotation $\theta_{\text {out }}$ with respect to the in- 


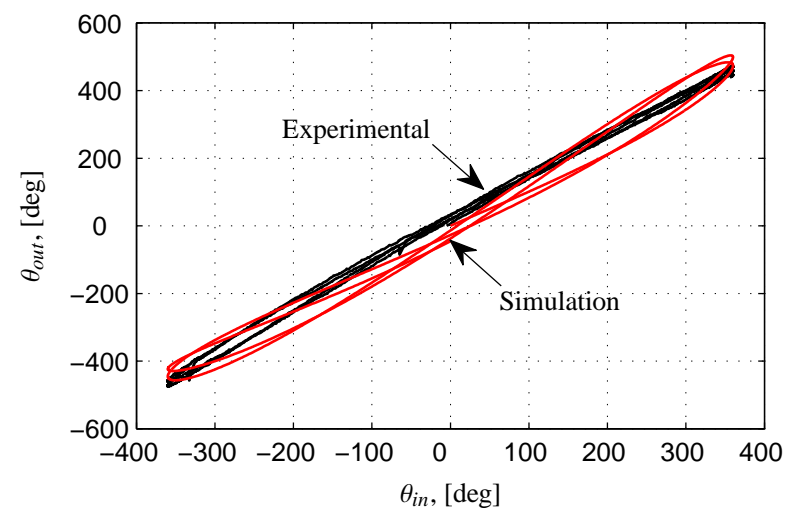

Fig. 4.26: Comparison of the rotation of the distal end $\theta_{\text {out }}$ with the simulation result for the given input rotation at the proximal end $\theta_{\text {in }}$

put rotation $\theta_{\text {in }}$ from the experiment and the simulation. The range of motion obtained from the experiment and the simulation are of the same order. The simulation result also showed an overshoot of the order of 100 degrees at the end of each stroke. However, there is significant difference in the experimental and simulation result. The simulation result shows the initial lag and the recovery. This is not evident in the experimental result. Nonetheless, the model predicts the range of motion in the same order of magnitude. The deviation in the result may be accounted for the initial curvature or unequal bending rigidity, which is not accounted for in the model. Characteristic plots can be obtained for various cases using the developed model. These characteristic plots can be used to estimate the unknown parameters and can be used in the model to further refine it.

\subsection{Discussion}

The assumption that the curved tube is rigid and the geometry of the tube is not changing due to the interaction with the instrument is generally valid because the endoscope is supported by the surrounding organs or part of the anatomy where it is inserted. Nonetheless, the interaction of the instrument tip with the tissue can exert such a high force on the endoscope that the endoscope cannot be assumed to be rigid anymore. Therefore, a flexible model for the tube will be required to address such interactions. The current model is limited to the interaction with the curved rigid tube only, but it can be extended to account for an elastic tube. 
The simulations for the rotation were rather computationally heavy. Therefore, a higher value of angular rotation is used for the simulation.

Whipping action of the instrument is not desired as it makes the instrument uncontrollable and can be dangerous in surgical applications. However, the presence of an initial curvature cannot be ignored because of manufacturing limitations. The model can be used to investigate the dependence of various parameters on the onset of whipping motion.

\subsection{Conclusion}

A general 3-D flexible multibody model of the instrument was set up. The contact model and the model of the curved rigid tube was appropriately defined. The effect of various parameters in motion and force transmission was studied through various simulations in translation and rotation.

The simulations for the fine manipulation in translation show the stick-slip behavior clearly when the input velocity changes direction. However, the magnitude of the stick-slip is greatly reduced when the distal end is preloaded in tension. The motion hysteresis is reduced from $2.8 \mathrm{~mm}$ to $0.6 \mathrm{~mm}$. For the unloaded case, the output motion of the distal end is oscillatory during the forward motion. However, the motion is smoother during the retraction. For the preloaded case, the output motion of the distal end is smoother during the both strokes of motion.

The ratio of the input force at the proximal end to the spring load at the distal end is compared with the capstan equation result. The simulation results show a deviation of $1.8 \%$ in the estimation of input force. The bending rigidity of the instrument and the magnitude of the axial load applied at the distal end have no significant effect on the force transmission for the given configuration.

The simulations of fine manipulation in rotation show a motion hysteresis of the order of 0.4 degree for the unloaded case. However, the motion hysteresis in rotation increases considerably with different flexural rigidity along the two principal axes and with a pre-curvature. An increased difference in the flexural rigidity along the principal axes and an increased value of pre-curvature result in a whipping action.

Measurements of the input force shows a deviation of the order of $1 \%$ from the analytical solution obtained from the capstan equation. The measured output rotation of the distal end with respect to the input rotation of the proximal end is in agreement with the simulation result.

Now we have a general 3-D model of a flexible instrument in a curved rigid 
tube, which can be used to study the effect of various parameters under different scenarios and for different geometric configurations for both in translation and rotation.

\section{Acknowledgment}

This research is funded with the TeleFLEX project, number PID07038, by the Dutch Department of Economic Affairs, Agriculture and Innovation and the Province of Overijssel, within the Pieken in de Delta (PIDON) initiative. 


\section{IMPROVED FORCE TRANSMISSION OF a Flexible Surgical Instrument by Combining Input Motion}

The force transmission of a flexible instrument through an endoscope is considerably deteriorated due to friction between the contacting surfaces. Friction force along the axial direction can be reduced by combining the translation motion input with rotation. A ratio $\zeta$ is defined to measure the reduction in the friction force along the axial direction due to the combined motion input at the proximal end of the instrument. An analytical formula is derived that shows the reduction in the friction force for the combined motion input. A flexible multibody model is set up and various simulations are performed with different motion inputs. The simulation result showed a reduction of $80 \%$ in the value of $\zeta$ in accordance with the analytical result for the given velocity ratio. Several experiments are performed with constant translation motion input combined with constant and sinusoidal rotational motion input. A maximum reduction of $84 \%$ is obtained in the value of $\zeta$ against a reduction of $89 \%$ calculated analytically. The knowledge of force transmission with a combination of motions can be used to increase the force fidelity of a flexible instrument in applications like robotic surgery with a flexible instrument.

Key words: friction alleviation, force transmission, flexible instrument, combined motion

J.P. Khatait, D.M. Brouwer, R.G.K.M. Aarts, \& J.L. Herder (2013). Improved Force Transmission of a Flexible Surgical Instrument by Combining Input Motion, Precision Engineering, Draft submitted, Under review. 


\subsection{Introduction}

The current robotic surgical systems have revolutionized the field of minimally invasive surgery (MIS) by dexterity enhancement, navigation and guidance, and improved visualization $[6,14,32,34]$. These systems often employ long rigid instruments. However, the application of flexible instruments becomes apparent and inevitable with new trend in surgery, i.e. flexible endoscopic surgery, Natural Orifice Transluminal Endoscopic Surgery (NOTES) and Single Incision Laparoscopic Surgery (SILS) [7,57]. Nonetheless, the surgeons are deprived of their touch feedback, often referred as haptic or force feedback, while performing robotic surgery using these instruments $[43,46,61]$. The addition of force feedback capabilities into MIS procedures provides an excellent opportunity to improve the quality of surgical procedures by reducing surgical errors and potentially increasing patient safety.

However, the frictional force causes disturbances to haptic sensations. The amount of haptic feedback may be enhanced by reducing the frictional force [43]. Moreover, the reduction in friction force also leads to improved motion transmission [55]. A better estimation of instrument tip motion also helps in accurate control of thin flexible surgical instrument [3].

In our previous study [26,29], we studied the translational and rotational behaviour of a flexible instrument inside a curved rigid tube. The force transmission of a flexible instrument is deteriorated due to friction between the instrument and the tube. Frictional force limits the amount of force delivered at the distal end of the instrument and vice-versa. The advantage with the robot-assisted system is that it can go beyond the surgeon's hand movement and the proximal end of the instrument can be actuated differently in translation and rotation along the longitudinal axis of the instrument. It is known that by combining the rotation motion while simultaneously applying force in the axial direction, an object can be easily pulled out, like a cork from a wine bottle. Therefore, we hypothesized that force transmission of a flexible surgical instrument can be improved by combining the rotation motion with the axial motion.

The objective of this paper is to study the effect of combined motion on the force transmission of the flexible instrument along the axial direction through a curved rigid tube. Firstly, a mathematical formula is derived to compare the force transmission along the axial direction for the combined motion with respect to the axial motion only. The ratio of the circumferential and the axial velocities at the contact point of the instrument due to the rotation and the translation input motion is defined. The effect of the velocity ratio on the force 
transmission is studied. The details of the analytical formulation are described in Section 5.2.1. Secondly, a flexible multibody model is set up to perform simulations for combined motion. The details of the model are described in Section 5.2.2. Thereafter, various experiments are performed to validate the analytical and simulation results. Section 5.2.3 describes the experimental set-up. The simulation and experimental results are discussed in Section 5.3. Finally, the discussion and the conclusion are presented in Section 5.4 and Section 5.5 respectively.

\subsection{Methods}

The effect of the combined motion on the force transmission is studied for a flexible instrument traversing through a curved rigid tube. A flexible wire of circular cross-section with diameter $d_{w}$ is used as a flexible instrument for the analysis. The wire is inserted through a curved rigid tube with a wrap angle $\alpha$. Figure 5.1 shows the configuration of the wire inside the tube. The input side is the proximal end of the wire. The translation motion input $x_{i n}$ is combined with the rotation motion input $\theta_{i n}$. The reaction force at the proximal end is the input force $F_{\text {in }}$. The output side, the distal end of the wire, is attached to a spring. The force applied at the distal end is the output force $F_{\text {out }}$. The output motions at the distal end are $-x_{\text {out }}$ in translation and $\theta_{\text {out }}$ in rotation.

A mathematical formula is derived for the reduction in the friction force along the axial direction due to the combined motion input. The force transmission of a flexible wire through a curved rigid tube is analyzed using the capstan equation. The ratio of the input and output forces is compared for the combined motion with that of the translation motion only. The details of the analytical formulation are described in Section 5.2.1. A case study is formulated and analyzed for different values of the combined motion. Analytical results provide a basic insight into the force transmission of a flexible wire due to the combined motion.

A flexible multibody model was developed to study the motion and force transmission of a flexible instrument inside a curved rigid tube [26, 29]. The model includes the mechanical properties of the instrument and that of the curved rigid tube, and also the contact properties. A flexible multibody is set up and described in detail in Section 5.2.2. Various simulations are performed and the results are compared with the analytical results. The simulation results are discussed in Section 5.3.

For the experimental validation, we performed various experiments using 


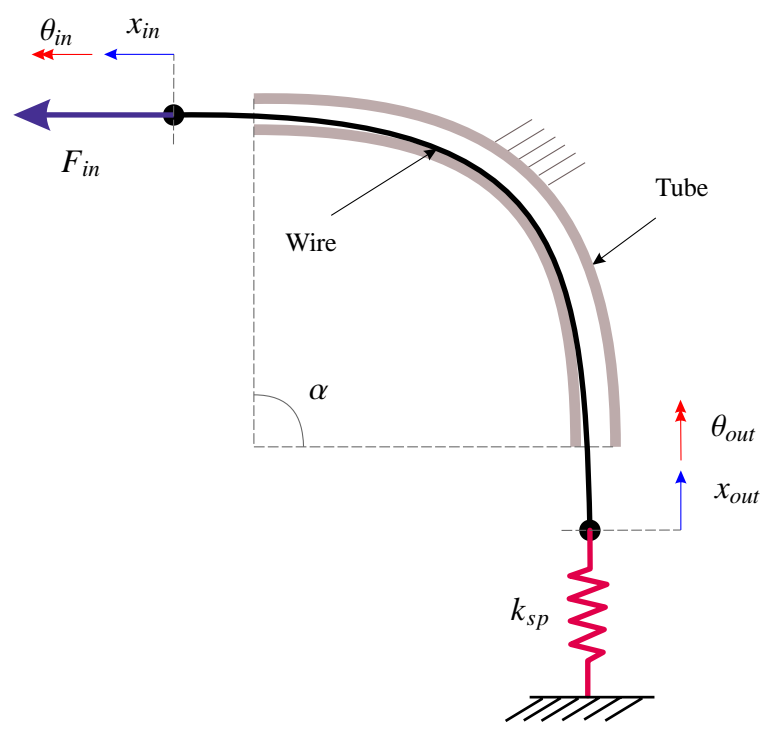

Fig. 5.1: A flexible wire inside a curved rigid tube. The distal end is attached to a spring $k_{s p}$. The input motion is applied at the proximal end.

flexible wire inside a curved rigid tube. An experimental set-up was designed to study the motion and force transmission of a flexible instrument inside a curved rigid tube [30]. The set-up provides the input motion to the proximal end and measures the forces of interaction between the wire and the tube. The experimental set-up is described in Section 5.2.3. The experimental results are discussed later in Section 5.3.

\subsubsection{Analytical Formulation}

The translation motion input at the proximal end results into sliding velocity $v_{a}$ along the axial direction. Likewise, the rotation motion input at the proximal end produces sliding velocity $v_{c}$ along the circumferential direction at the point of contact. If $\omega$ is the angular velocity along the axial direction and $d_{w}$ is the diameter of the instrument, the instantaneous velocity at the point of contact along the circumferential direction is given by $v_{c}=\omega d_{w} / 2$.

Assuming the input motions, translation and rotation, at the proximal end are conveyed uniformly along the length of the wire, the resultant sliding velocity at the point of contact on the tangent plane is given by

$$
\begin{aligned}
v_{t} & =\left[v_{a}{ }^{2}+v_{c}{ }^{2}\right]^{1 / 2} \\
\tan \phi & =v_{c} / v_{a}
\end{aligned}
$$




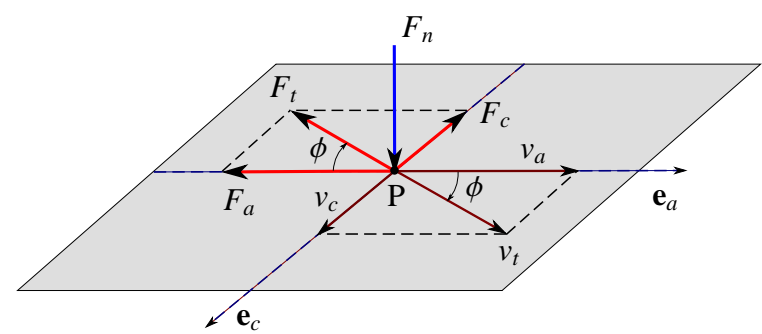

Fig. 5.2: Friction force at contact point $P$. The unit vectors along the axial direction $\mathbf{e}_{a}$ and the circumferential direction $\mathbf{e}_{c}$ constitute the tangent plane at the point of contact. The resultant tangential velocity $v_{t}$ makes an angle $\phi$ with the unit vector $\mathbf{e}_{a}$. The resultant friction force $F_{t}$ acts in the opposite direction of $v_{t}$.

where the angle $\phi$ is the angle the resultant sliding velocity $v_{t}$ makes with the unit vector $\mathbf{e}_{a}$ along the axial direction. The velocity components along the axial and circumferential directions on the tangent plane at the point of contact $\mathrm{P}$ are shown in Fig. 5.2.

\section{Reduction in friction force along the axial direction}

As the friction force acts in the opposite direction of the resultant sliding velocity, the resultant friction force also makes an angle $\phi$ with the unit vector $\mathbf{e}_{a}$ along the axial direction. The friction force components along the axial and circumferential directions are shown in Fig. 5.2. It is evident from the figure that for a given velocity $v_{t}$ the friction force along the axial direction is the maximum for $\phi=0$ and $F_{a}=F_{t}$. On the other hand, the friction force along the axial direction is the minimum for $\phi=\pi / 2$ and $F_{a}=0$. In general, the friction force along the axial direction is given by

$$
F_{a}=F_{t} \cos \phi .
$$

If we assume the Coulomb friction force model, for the given normal reaction force $F_{n}$ acting at the contact point $\mathrm{P}$, the friction force $F_{t}$ is given by

$$
F_{t}=\mu F_{n}
$$

where $\mu$ is the coefficient of friction between the two contacting surfaces.

Therefore, if the translation motion is combined with the rotation motion, the friction force along the axial direction can be rewritten as

$$
F_{a}=\mu F_{n} \cos \phi .
$$


Hence, the effective coefficient of friction $\mu_{\text {eff }}$ along the axial direction in the case of combined motion is

$$
\mu_{\mathrm{eff}}=\mu \cos \phi .
$$

The reduction in the friction force along the axial direction due to the combined motion is given by

$$
\Delta F_{a}=(1-\cos \phi) F_{t} .
$$

Therefore, by combining the motion, the friction force along the axial direction can be reduced. A reduction in the overall friction force along the axial direction also leads to reduced motion hysteresis in translation [55].

\section{Force transmission due to the combined motion}

For the wire in curved tube as shown in Fig. 5.1, the force transmission in the translation motion can be compared with the capstan equation. The wellknown relationship between the output and input forces on the both sides of a flexible string wound around a circular body is given by the capstan equation [23]. If $F_{\text {in }}$ and $F_{\text {out }}$ are the forces on the input and output sides, the capstan equation can be written as

$$
\frac{F_{\text {in }}}{F_{\text {out }}}=e^{ \pm \mu \alpha}
$$

where $\mu$ is the coefficient of friction between the two contacting surfaces, and $\alpha$ is the wrap angle. Here, the sign is positive when the wire is retracting and it is negative when the wire is moving forward.

If there is only translation motion along the axial direction, the logarithm of the force ratio can be written as

$$
\ln \left(\frac{F_{\text {in }}}{F_{\text {out }}}\right)_{\text {trans }}= \pm \mu \alpha .
$$

The absolute value of the logarithm of the force ratio remains constant for a given configuration of the wire and the tube and is equal to $\mu \alpha$. The sign depends on the direction of motion of the wire.

In the case of the combined motion, the logarithm of the force ratio is given by

$$
\ln \left(\frac{F_{\text {in }}}{F_{\text {out }}}\right)_{\text {comb }}= \pm \mu_{\text {eff }} \alpha .
$$


Let us define $\zeta$ as a ratio of the logarithm of the force ratio for the combined motion and the logarithm of the force ratio for the translation motion to compare the effect of the combined motion on the force transmission along the axial direction, that is

$$
\zeta=\frac{\ln \left(\frac{F_{\text {in }}}{F_{\text {out }}}\right)_{\text {comb }}}{\left|\ln \left(\frac{F_{\text {in }}}{F_{\text {out }}}\right)_{\text {trans }}\right|} .
$$

Here, we use the absolute value in the denominator for the comparison. From Eqn. (5.9), Eqn. (5.10) and Eqn. (5.6), Eqn. (5.11) can be rewritten as

$$
\begin{aligned}
\zeta & =\frac{ \pm \mu_{\mathrm{eff} \alpha}}{\mu \alpha} \\
& = \pm \cos \phi
\end{aligned}
$$

The ratio $\zeta$ is used to calculate the force transmission along the axial direction. As $\phi \rightarrow \pi / 2$, the value of $\zeta$ approaches towards 0 . Correspondingly, the force transmission along the axial direction will be the maximum. In this case, the friction force will be acting along the circumferential direction of the wire, and the force transmission along the axial direction will be maximized. However, As $\phi \rightarrow 0$, the value of $\zeta$ approaches towards 1 . The force transmission along the axial direction, in this case, will be the minimum. The friction force will act along the axial direction of the wire, and will limit the force transmission along the axial direction.

For all angles $\phi$, it follows from Eqn. (5.2) and Eqn. (5.13) that $\zeta$ can be expressed in terms of velocity ratio $v_{c} / v_{a}$ as

$$
\zeta= \pm \frac{1}{\sqrt{1+\left(v_{c} / v_{a}\right)^{2}}} .
$$

It is apparent from Eqn. (5.14) that the magnitude of the ratio $\zeta$ decreases with increasing velocity ratio $v_{c} / v_{a}$ and, therefore, the force transmission improves with the increased velocity ratio.

\section{Analytical results}

We analyzed the effect of the combined motion on the force transmission along the axial direction. From Eqn. (5.14), we observe that the ratio $\zeta$ depends on the velocity ratio $v_{c} / v_{a}$. In order to get different velocity ratio, a constant axial velocity is combined with a sinusoidal rotation input at the proximal end. 
Let us consider a guide wire inserted into a curved rigid tube as illustrated in Fig. 5.1. The distal end of the guide wire is attached to a spring with a linear stiffness $k_{\mathrm{sp}}$. The input motion is described at the proximal end both in the translation and rotation. The coefficient of friction between the contacting surfaces is given by $\mu$.

It is assumed that the guide wire is loaded in tension all the time. A constant velocity $v_{\text {in }}$ is defined along the axial direction. A sinusoidal rotation motion of amplitude $A$ and the frequency $f$ is also defined at the proximal end such that the initial angular displacement and the velocity are zero, and is given by

$$
\begin{aligned}
& \theta_{\text {in }}=A \cos (2 \pi f t)-A \\
& \dot{\theta}_{\text {in }}=-2 \pi f A \sin (2 \pi f t) .
\end{aligned}
$$

Therefore, the velocity components along the axial and circumferential directions are given by

$$
\begin{aligned}
& v_{a}= \pm v_{\text {in }} \\
& v_{c}=\dot{\theta}_{\text {in }} d_{w} / 2 .
\end{aligned}
$$

The axial velocity has a constant magnitude, but the sign is reversed to limit the range of the axial motion. It is assumed that the guide wire can freely rotate along the axial direction and the spring does not constrain the guide wire in rotation.

\section{Case study: constant $v_{a}$ with sinusoidal $v_{c}$}

Input motion profile both in translation and rotation are defined to analyze the effect on the ratio $\zeta$. A constant translation velocity $v_{a}=5 \mathrm{~mm} / \mathrm{s}$ is defined along the axial direction. Its direction is reversed every $1 \mathrm{~s}$. A sinusoidal motion is defined in rotation with different amplitudes $A=\pi, 2 \pi$, and $3 \pi$ radians and a frequency $f=1 \mathrm{~Hz}$ (given by Eqn. (5.16)). A guide wire of diameter $d_{w}=0.8 \mathrm{~mm}$ is considered. The corresponding velocities along the circumferential direction $v_{c}$ are calculated (Eqn. (5.18)). Figure 5.3 shows the velocity profiles in the axial and circumferential directions. For $t<1.75 \mathrm{~s}$, there is no rotation motion defined. The velocity along the circumferential direction $v_{c}$ is triggered after $t=1.75 \mathrm{~s}$.

The value of the ratio $\zeta$ is calculated for different values of the axial velocity $v_{a}$ and the circumferential velocity $v_{c}$ using Eqn. (5.14) and shown in Fig. 5.4. For $t<1.75 \mathrm{~s}$, as there is no rotation motion, the value of the ratio $\zeta$ changes 


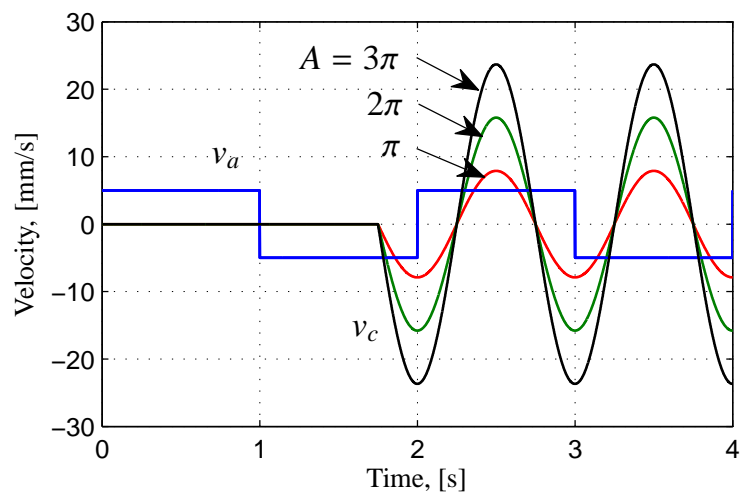

Fig. 5.3: Velocity profile of the input motion in translation and rotation. The velocity along the axial direction $v_{a}$ is combined with the velocity along the circumferential direction $v_{c}$ after $t=1.75 \mathrm{~s}$. The velocities along the circumferential direction are shown for different amplitude $A$ [rad] and frequency $f=1 \mathrm{~Hz}$.

to \pm 1 as the direction of motion changes. However, after $t=1.75 \mathrm{~s}$, the magnitude of the ratio $\zeta$ starts decreasing from 1 as the magnitude of the sinusoidal rotation motion starts increasing. The maximum decrease in the magnitude of the ratio $\zeta$ occurs when the circumferential velocity is maximum for the given axial velocity. As the circumferential velocity approaches zero, the magnitude of the ratio $\zeta$ also approaches towards 1 . In the subsequent periodic behaviour, the frequency of the sine function can be recognized.

Here, we observed that when the circumferential velocity $v_{c}$ is zero, the friction force acts completely along the axial direction and thus limits the force transmission. However, as the magnitude of the circumferential velocity $v_{c}$ increases, the magnitude of the ratio $\zeta$ decreases and it reaches to the minimum for the maximum value of $v_{c}$.

We observed a significant decrease in the friction force in the axial direction if the velocity along the axial direction $v_{a}$ is combined with the velocity along the circumferential direction $v_{c}$. From the chosen case study for the analysis, the ratio $\zeta$ reduces to 0.2 . The reduction in the friction force will increase the force fidelity of a flexible guide wire in a curved rigid tube. The backlash-associated with the friction-can be greatly reduced. In our analytical study, we did not consider the dynamic effects due to the stiffness or the inertia properties of the guide wire. We assumed that the wire is infinitely rigid in translation and rotation. We also assumed that the wire is compliant in bending. Nonetheless, these assumptions simplify the overall problem and help in deriving the basic governing equations.

The stiffness and inertia properties of the guide wire can have a detrimental 


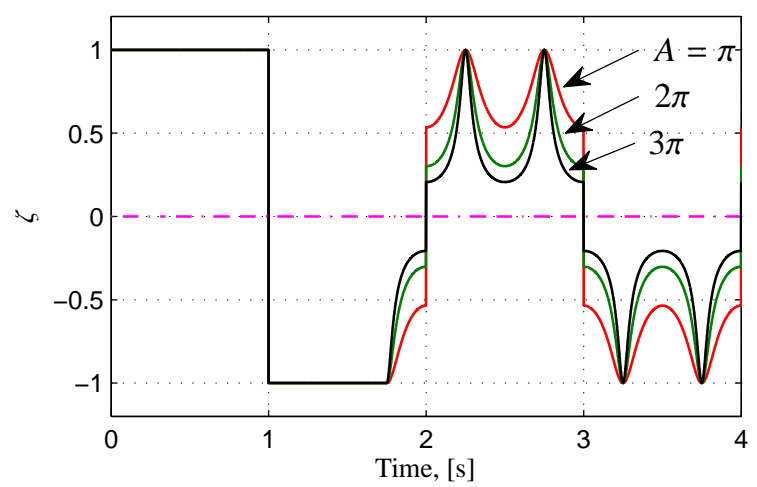

Fig. 5.4: The ratio $\zeta$ for the combined motion as the velocity ratio changes. The sinusoidal rotation motion is combined with the constant axial motion after $t=1.75 \mathrm{~s}$. Several plots of the ratio $\zeta$ are shown for sinusoidal rotation motion of different amplitudes $A$ [rad] and frequency $f=1 \mathrm{~Hz}$.

effect on the force transmission and can pose limitations to the analytical results. Therefore, the analytical results are validated with the flexible multibody model and with the experiments.

\subsubsection{Flexible Multibody Model of an Instrument and a Tube}

We developed a flexible multibody model to study the behavior of a flexible instrument inside a curved rigid tube. The modeling task requires three key components-a finite element model of the instrument, a model of the curved rigid tube, and the contact model to define the interaction between the instrument and the tube.

In general, a flexible instrument deforms mainly in bending and torsional directions due to the loading conditions at the instrument tip, the motion and force input at the proximal end, and the interaction of the instrument with the tube. The flexible instrument is modeled as a series of interconnected flexible beam elements. Each beam element has six deformation modes defined-one mode for the elongation, one mode for the torsion, and four modes for the bending deformations of the element [21,22]. Figure 5.5 shows the flexible multibody model of the instrument and the curved rigid tube. The encircled number, (1), represents the $n^{\text {th }}$ beam element. The nodes are represented by the numbers.

The curved rigid tube is modeled as a circular tube of uniform cross-section. The shape of the tube is defined by the centerline of the tube. The centerline is defined by a straight line, a circular arc, a Bézier curve, or a combination of these. Different geometric shapes are connected together to define the en- 


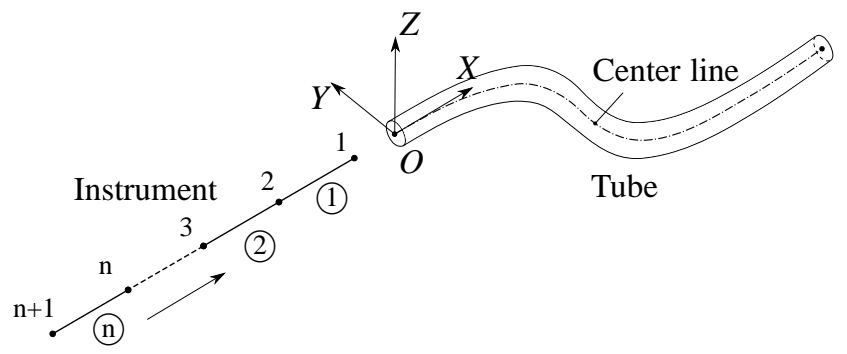

Fig. 5.5: Flexible multibody model of a instrument and of a curved rigid tube

tire length of the curved tube. The contact model includes the interaction in the normal and tangential directions at the various nodes. Wall stiffness and damping are defined to calculate the interaction in the normal direction. Friction between the instrument and the tube is defined to calculate the interaction in the tangential direction. The details of the developed model can be referred to $[26,28,29]$.

A flexible multibody model is set up to validate the analytical results. The effect of the velocity components-along the axial and circumferential directionson the force transmission is further investigated.

\section{Description of the Model}

A flexible instrument is defined by a stainless steel wire of circular crosssection of $0.8 \mathrm{~mm}$ diameter. The undeformed wire is straight and has no precurvature. The entire length of the wire is defined by interconnected spatial beam elements. A curved rigid tube is defined by an arc of radius of curvature $300 \mathrm{~mm}$ and a wrap angle $\alpha=90^{\circ}$. The inner diameter of the tube is $4.0 \mathrm{~mm}$. Two straight tubes of the same inner diameter are defined at the entry and exit of the curved tube. The wire inside the circular section is defined by 10 equal length spatial beam elements. A section of $130 \mathrm{~mm}$ and $200 \mathrm{~mm}$ long wire are defined in the straight sections of the tube at the entry and at the exit respectively. Figure 5.6 shows the configuration of the model.

\section{Simulation for the combined motion}

The model is set up for the simulation. The instrument is assumed straight in the beginning. The initial deformations of the elements are obtained analytically and prescribed in the beginning to place the instrument along the centerline of the defined curved rigid tube. The data used for the model and 


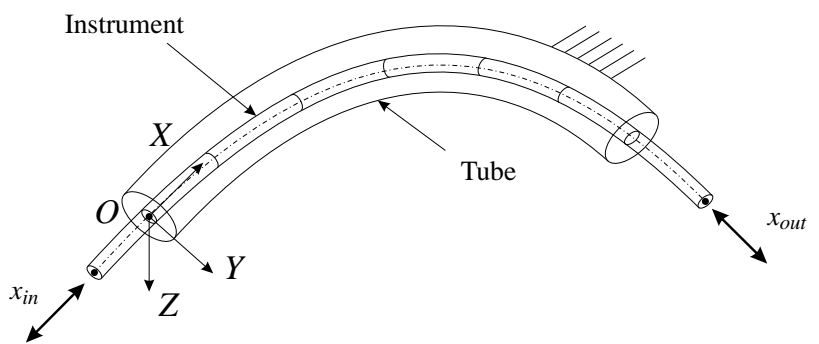

Fig. 5.6: Configuration of the flexible multibody model of the instrument and of the curved rigid tube used for the simulation. The instrument is defined by interconnected spatial beam elements.

Table 5.1: Data used for the simulations

\begin{tabular}{lrr}
\hline Description & Values & Unit \\
\hline Density, $\rho$ & 7.8 & $10^{3} \mathrm{~kg} / \mathrm{m}^{3}$ \\
Young's modulus, $E$ & 200 & $10^{9} \mathrm{~N} / \mathrm{m}^{2}$ \\
Shear modulus, $G$ & 79 & $10^{9} \mathrm{~N} / \mathrm{m}^{2}$ \\
Mass/length, $\rho A$ & 3.92 & $10^{-3} \mathrm{~kg} / \mathrm{m}$ \\
Flexural rigidity, $E I$ & 4.02 & $10^{-3} \mathrm{~N} \mathrm{~m}$ \\
Torsional rigidity, $G J$ & 3.18 & $10^{-3} \mathrm{~N} \mathrm{~m}^{2}$ \\
\hline Size of the transition zone & 0.5 & $\mathrm{~mm}$ \\
Wall stiffness, $k$ & 2.0 & $10^{4} \mathrm{~N} / \mathrm{m}^{2}$ \\
Wall damping, $c_{w}$ & 10.0 & $\mathrm{Ns} / \mathrm{m}^{2}$ \\
Velocity coefficient, $c_{v}$ & 2.0 & $10^{3}(\mathrm{~m} / \mathrm{s})^{-1}$ \\
Friction coefficient, $\mu$ & 0.157 & \\
\hline
\end{tabular}

simulation are given in Table 5.1. The load at the distal end is applied through a linear spring of stiffness $k_{s p}=198.3 \mathrm{~N} / \mathrm{m}$ and an initial extension of $12.5 \mathrm{~mm}$. A lumped mass and inertia are added at the distal end to account for attachments used for measurement (see Section 5.2.3). The mass and inertia properties are given in Table 5.2.

An input motion profile is defined at the proximal end of the instrument in both directions - translation and rotation-as shown in Fig. 5.3 and outlined in Section 5.2.1. In the simulations, the amplitude is $A=2 \pi \mathrm{rad}$, and the frequency is $f=1 \mathrm{~Hz}$. The simulation is performed for $t=6 \mathrm{~s}$. The rotation motion is triggered at $t=1.75 \mathrm{~s}$ so that when the instrument reverses the direction at $t=2 \mathrm{~s}$, the circumferential velocity $v_{c}$ due to rotation reaches to the maximum. 
Table 5.2: Mass properties of the attachment

\begin{tabular}{lrrr}
\hline Parameter & Value & Unit \\
\hline Rotational inertia & $J_{x}$ & 1.36 & $10^{-5} \mathrm{~kg} \mathrm{~m}^{2}$ \\
& $J_{y}$ & 17.47 & $10^{-5} \mathrm{~kg} \mathrm{~m}^{2}$ \\
& $J_{z}$ & 17.67 & $10^{-5} \mathrm{~kg} \mathrm{~m}^{2}$ \\
\hline Mass & $m$ & 0.100 & $\mathrm{~kg}$ \\
\hline
\end{tabular}

The input force $F_{\text {in }}$ and the output force $F_{\text {out }}$ are calculated during the simulation. The input force $F_{\text {in }}$ is the force acting at the proximal end and is equal to the reaction force. The output force $F_{\text {out }}$ is the force acting at the distal end and is equal to the spring force. The extension in the spring $x_{\text {extn }}$ is calculated from the displacement at the distal end. Therefore, the output force $F_{\text {out }}=k_{s p} x_{\text {extn }}$. The force ratio $F_{\text {in }} / F_{\text {out }}$ is calculated for the entire motion range. The ratio $\zeta$ is calculated further using Eqn. (5.11). The details of the simulation results are presented in Section 5.3.

The coefficient of friction $\mu$ between the contacting surfaces is assumed constant. The Coulomb friction force model is used in the simulation. The value of $\mu$ does not change with the magnitude of sliding velocity.

\subsubsection{Experiments}

Various experiments are performed to validate the analytical and simulation results. We used the experimental set-up previously designed to study the motion and force transmission of a flexible instrument inside a curved rigid tube [30]. A brief description of the set-up is given in the following section.

\section{Description of the experimental set-up}

The experimental set-up consists of three basic modules: Actuation module (AM), Force sensing module (FSM), and Tip motion measurement module (T3M). Figure 5.7 shows the experimental set-up, indicating the three modules together with the key components.

The AM provides the actuation in 2-DOFs, i.e. translation and rotation along the axial axis of the instrument at the proximal end. The FSM enables all 6DOFs measurement of forces and torques arising from the interaction of the instrument with the curved rigid tube. The T3M measures the translation and rotation of the distal end of the instrument along the axial direction. The details of the design and the evaluation of the set-up can be referred to [30]. 


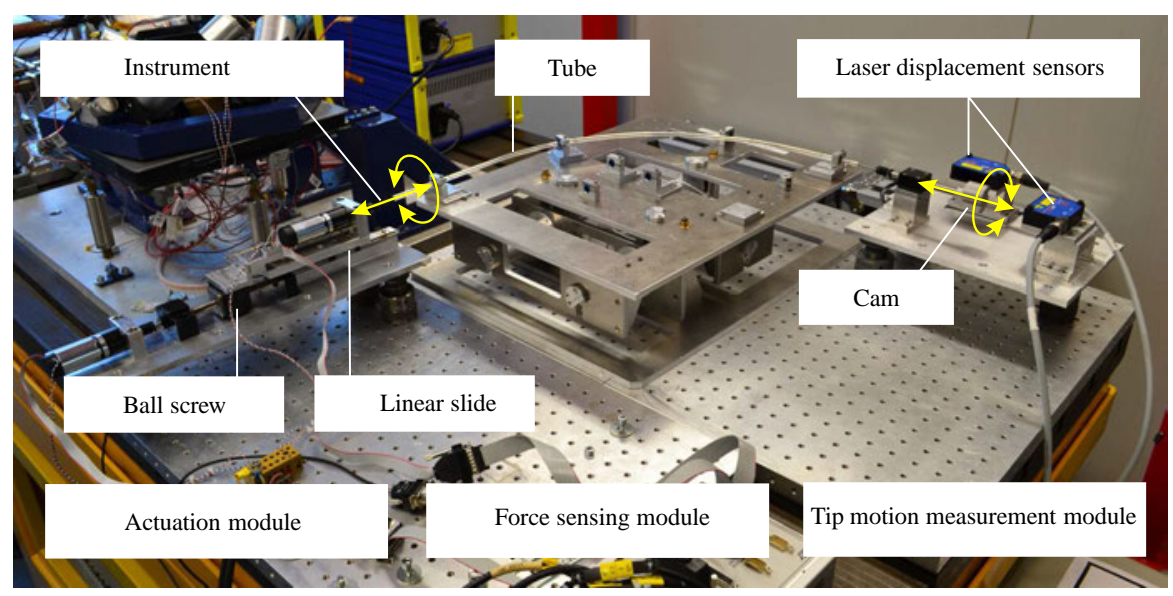

Fig. 5.7: Experimental set-up with 2-DOFs actuation module (AM), 6-DOFs force sensing module (FSM) and 2-DOFs tip motion measurement module (T3M). The AM provides actuation at the proximal end. The FSM measures all the six components of the interaction force between the instrument and the tube. The T3M performs contactless measurement of the distal end motion.

The combined motion is effected through the translation and rotation motion of the AM independently. The motions along the two DOFs are measured by the encoders. The proximal end of the wire is attached to the AM at the rotation axis. The motion of the distal end of the wire is measured via the cam through two laser displacement sensors. The forces acting at the ends of the wire are measured from the reaction forces measured by the FSM.

A force is applied to the distal end along the axial direction through a spring. The spring is attached to the base through a string. The configuration allows the loading of the wire in the axial direction. As the string is extremely compliant in bending and torsion, the rotation motion can be combined with the translation motion without loading the wire in torsion. Figure 5.8 shows the details of the attachment to the distal end.

\section{Configuration of the guide tube and wire}

For the guide tube, we used a stainless steel tube of outer diameter of $6 \mathrm{~mm}$ and inner diameter of $4 \mathrm{~mm}$. A total length of $800 \mathrm{~mm}$ was chosen. The tube was bent to $90^{\circ}$ arc of radius of curvature $300 \mathrm{~mm}$. There were straight sections left at both the ends. The input side of the tube was aligned to the AM and the output side of the tube was aligned to the T3M. The tube was rigidly attached to the top plate of the FSM. 


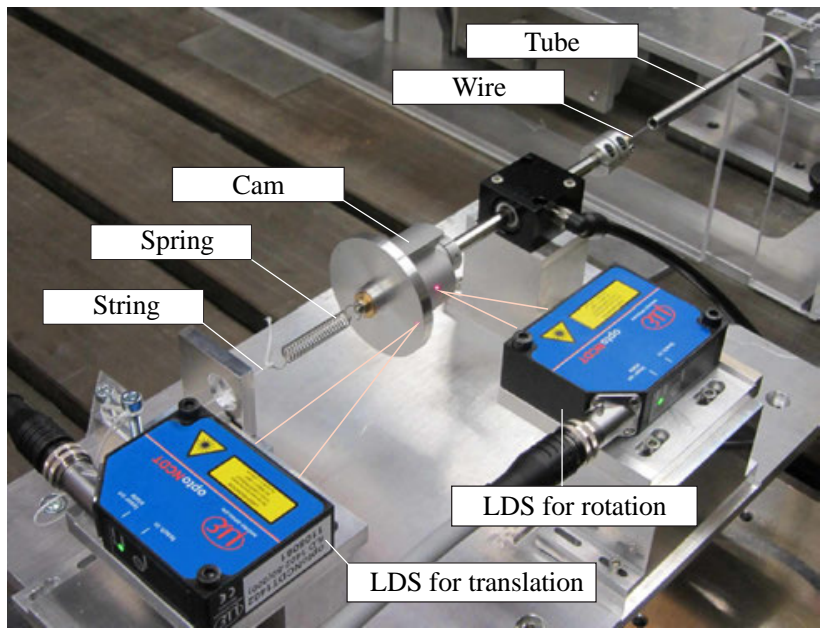

Fig. 5.8: Experimental set-up with the spring attached to the distal end of the wire. One end of the spring is attached to the base through a string allowing loading in axial direction only.

We chose a stainless steel wire of diameter $0.8 \mathrm{~mm}$ and a total length of $880 \mathrm{~mm}$. The wire has no precurvature. The wire is inserted into the tube and the two ends are attached to the AM and the T3M. The axial load is applied through the spring and is attached to the base through a string. The extension of the spring is adjusted so that there is always a tensile load acting on the wire throughout the range of motion in the axial direction.

\subsection{Results}

The effect of the combined motion is studied through various simulations and experiments. The simulation and experimental results are compared with the analytical result. The combined motion results in a reduction in the force transmission in the axial direction. The value of the ratio $\zeta$, defined by Eqn. (5.11), gives an indication of the amount of reduction in the friction force along the axial direction. The combined motion is presented in terms of the velocity ratio $v_{c} / v_{a}$. The value of the ratio $\zeta$ is studied for different values of the velocity ratio. The simulation and experimental results are presented in the following sections.

\subsubsection{Simulation Results}

Simulation results are presented with respect to the given input motion. The input force $F_{\text {in }}$ and output force $F_{\text {out }}$ are calculated and shown in Fig. 5.9. 


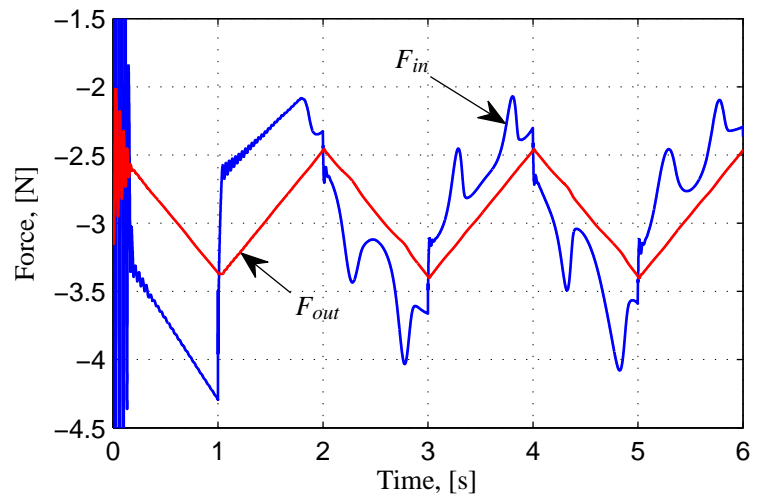

Fig. 5.9: Comparison of the input and output forces with and without rotation motion combined with the translation motion. The sinusoidal rotation motion is combined with the constant axial motion after $t=1.75 \mathrm{~s}$.

We can observe the transients in the beginning. The output force $F_{\text {out }}$ varies linearly as it depends on the extension of the spring. Initially, as there is only translation motion along the axial direction, the friction force will be entirely acting along the axial direction. When the direction of motion changes, the direction of friction force also changes. The corresponding jump in the input force $F_{\text {in }}$ can be observed at $t=1.0 \mathrm{~s}$. The rotation motion is combined with the translation motion after $t=1.75 \mathrm{~s}$. The corresponding changes in the input force $F_{\text {in }}$ can be observed. As the magnitude of the circumferential velocity $v_{c}$ increases, the friction force along the axial direction decreases. Therefore, the difference in the input force $F_{\text {in }}$ and the output force $F_{\text {out }}$ decreases. Inversely, when the magnitude of the circumferential velocity $v_{c}$ approaches zero, the friction force acts entirely along the axial direction. Therefore, the difference between the forces is the maximum.

Effect of the velocity ratio on the ratio $\zeta$ The ratio $\zeta$ is calculated to compare the effect of the combined motion on the force transmission (see Eqn. (5.11)). The logarithm of the force ratio for the combined motion, $\ln \left(F_{\text {in }} / F_{\text {out }}\right)$, is calculated. The magnitude of the logarithm of the force ratio for the axial motion only is $\mu \alpha$. In this case, the value of $\mu$ is 0.157 and $\alpha$ is $\pi / 2$ rad. Figure 5.10 shows the corresponding plot of the ratio $\zeta$. Initially, the value of $\zeta$ is 1.0 as there is no rotation motion combined with the axial motion. However, as the translation motion is combined with the rotation motion, we can observe a decrease in the value of $\zeta$. The analytical value of the ratio $\zeta$ is calculated using Eqn. (5.14) as previously shown in Fig. 5.4 and also included in Fig. 5.10. The 


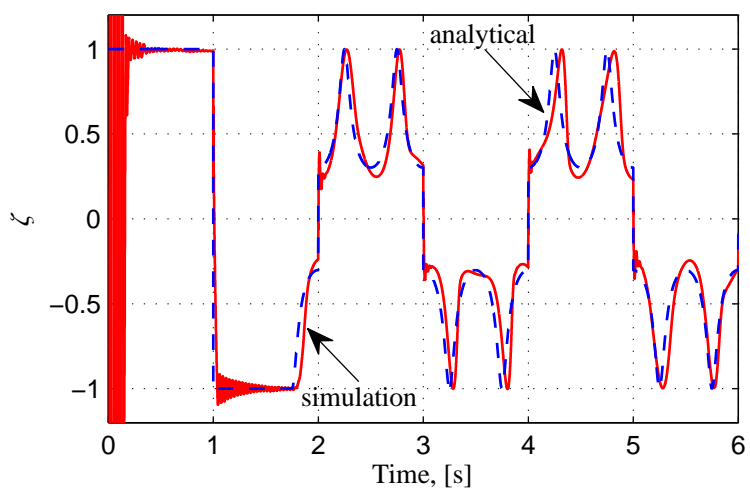

Fig. 5.10: Comparison of the value of the ratio $\zeta$ with and without rotation motion combined with the translation motion. The sinusoidal rotation motion is combined with the constant axial motion after $t=1.75 \mathrm{~s}$. Analytical result is shown by the dashed line (--).

simulation result shows a good correlation with the analytical result.

In order to study the effect of the velocity ratio further, different magnitudes of the sinusoidal rotation motion were combined with the translation motion. The magnitude of the axial velocity $v_{a}$ is $5 \mathrm{~mm} / \mathrm{s}$ for all the cases. The amplitude of the rotation motion is changed. The different values of $A$ used are $0.0,0.5 \pi, 1.0 \pi, 2.0 \pi$ and $3.0 \pi \mathrm{rad}$. The value of the frequency $f$ used is $1 \mathrm{~Hz}$. Figure 5.11 shows the plot of the ratio $\zeta$ for the combined motion for different amplitudes of rotation motion with respect to the input displacement $x_{i n}$ in axial direction. When the amplitude $A$ of the rotation motion is increased from $0.5 \pi$ to $3.0 \pi \mathrm{rad}$, the magnitude of the circumferential velocity $v_{c}$ also increases. As a result the ratio $\zeta$ decreases with increasing the amplitude $A$. The results are compared for one full stroke of motion, i.e. for $4.0 \mathrm{~s} \leq t \leq 6.0 \mathrm{~s}$ for clarity. The value of $\zeta$ remains at 1.0 for no rotation. It can be also observed that the oscillations are damped out quickly for higher values of the amplitude. A lag is also observed in the response.

The simulation results show that as the velocity ratio $v_{c} / v_{a}$ increases, the value of the ratio $\zeta$ decreases. A largest reduction of $80 \%$ in the value of $\zeta$ is observed in the case of a constant axial velocity $v_{a}=5 \mathrm{~mm} / \mathrm{s}$ combined with the sinusoidal rotation input of amplitude $A=3.0 \pi$ rad and frequency $f=1 \mathrm{~Hz}$. In this case, the value of $\zeta$ reduces from 1.0 to 0.2 , which is in agreement with the analytical result.

\subsubsection{Experimental Results}

Various experiments are performed to see the effect of the combined motion on the value of the ratio $\zeta$. Different velocity ratios $v_{c} / v_{a}$ are obtained by com- 


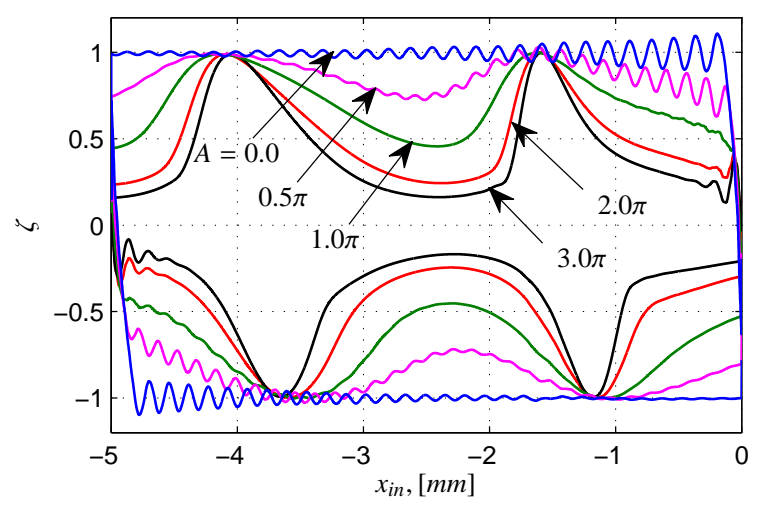

Fig. 5.11: Comparison of the value of the ratio $\zeta$ corresponding to constant axial velocity $v_{a}=5 \mathrm{~mm} / \mathrm{s}$ combined with the sinusoidal rotation input of different amplitudes $A$ [rad] and frequency $f=1 \mathrm{~Hz}$.

bining the constant translation $v_{\text {in }}$ with either the constant rotation input $\omega_{\text {in }}$ or with the sinusoidal rotation input. Both kinds of input motion for rotation are discussed in the following sections with respect to each translation input $v_{i n}$.

\section{Estimation of the value of $\mu$}

An experiment was set up to estimate the value of the coefficient of friction $\mu$ between the stainless steel wire and the tube. The wire is inserted into the curved rigid tube. The distal end is attached to the spring. A constant velocity, $v_{\text {in }}=0.5 \mathrm{~mm} / \mathrm{s}$, to-and-fro motion is given to the proximal end. The forces at the two ends of the wire are calculated.

The value of $\mu$ is calculated from the logarithm of the force ratio. From Eqn. (5.9),

$$
\left|\ln \left(\frac{F_{\text {in }}}{F_{\text {out }}}\right)\right|=\mu \alpha .
$$

The mean value, thus, calculated over the two cycles is 0.247 . For $\alpha=\pi / 2 \mathrm{rad}$, the value of $\mu$ is 0.157 . This is the value of $\mu$ used in the simulation.

\section{Constant translation $v_{\text {in }}$ with constant rotation $\omega_{\text {in }}$}

Experiments are performed for different velocity ratios by combining a constant translation input $v_{i n}$ with constant rotation input $\omega_{i n}$. The constant velocity input of $0.25,0.50$ and $1.0 \mathrm{~mm} / \mathrm{s}$ in translation are used for the experiments. The translation motion is combined with the constant angular velocity of 0 , $\pi / 3, \pi / 2$ and $\pi \mathrm{rad} / \mathrm{s}$. 
Case 1: $v_{\text {in }}=0.25 \mathrm{~mm} / \mathrm{s} \quad$ A constant velocity $v_{\text {in }}$ of $0.25 \mathrm{~mm} / \mathrm{s}$ is given to the proximal end of the wire. The motion is reversed after $20 \mathrm{~s}$ and the cycle is repeated. Initially, there is no rotational motion combined with the axial motion. The input and output forces are measured and the logarithm of the force ratio, $\ln \left(F_{\text {in }} / F_{\text {out }}\right)_{\text {trans }}$, is calculated. The experiment is repeated with the same axial motion combined with the rotational speed $\omega_{i n}=\pi / 2 \mathrm{rad} / \mathrm{s}$. The rotation motion is reversed after $10 \mathrm{~s}$ and the cycle is repeated. The input and output forces are measured and the logarithm of the force ratio, $\ln \left(F_{\text {in }} / F_{\text {out }}\right)_{\text {comb }}$, is calculated for the combined motion.

The ratio $\zeta$ is calculated using Eqn. (5.11) where the denominator is obtained from the mean value of $\left|\ln \left(F_{\text {in }} / F_{\text {out }}\right)_{\text {trans }}\right|$. Figure 5.12(a) shows the plot of the ratio $\zeta$ with respect to the input displacement. Here, we observed that when the translation motion is combined with the rotation motion, the value of the ratio $\zeta$ is reduced considerably. For a translation speed of $v_{i n}=0.25 \mathrm{~mm} / \mathrm{s}$ and rotation speed of $\pi / 2 \mathrm{rad} / \mathrm{s}$, the calculated value of angle $\phi$ is $68.3^{\circ}$. The value of the ratio $\zeta$ for the combined motion is also compared with the analytical result, $\cos \phi=0.37$. As the rotation motion changes direction every $10 \mathrm{~s}$, we observe an increase in the value of $\zeta$ at the end of stroke and also halfway. This is expected as there is no rotation momentarily and, therefore, the value of $\zeta$ approaches towards 1.0. However, the value of $\zeta$ reduces as soon as the rotation commences.

The value of $\zeta$ is calculated for another value of the angular rotation $\omega_{\text {in }}=$ $\pi / 3 \mathrm{rad} / \mathrm{s}$ combined with the axial motion $v_{i n}$. The value of $\zeta$ is also compared with the corresponding value of the analytical value, $\cos \phi$. The results are summarized in Tab. 5.3. The reduction in the ratio $\zeta$ with respect to the velocity ratio is observed in the experiments, although the experimental reduction is somewhat smaller than the analytical values. The rms values of $\zeta$ are also calculated for different velocity ratios and shown in Tab. 5.3. The rms values are relatively high due to velocity reversals in the rotational direction. The sudden change in the velocity also excites the higher modes and leads to oscillations, which is reflected in the rms values.

Case 2: $v_{\text {in }}=0.5 \mathrm{~mm} / \mathrm{s}$ In this case, a constant input velocity $v_{i n}=$ $0.5 \mathrm{~mm} / \mathrm{s}$ is given to the proximal end. The motion is reversed after $10 \mathrm{~s}$ and repeated. The translation motion is combined with different rotational speed $\omega_{i n}-0, \pi / 2$ and $\pi \mathrm{rad} / \mathrm{s}$. The rotation motion is reversed after every $5 \mathrm{~s}$. Figure 5.12(b) shows the plot of the ratio $\zeta$ for rotation input of $\omega_{\text {in }}=\pi \mathrm{rad} / \mathrm{s}$ and translation input of $v_{i n}=0.5 \mathrm{~mm} / \mathrm{s}$ with respect to the input displacement $x_{i n}$. 


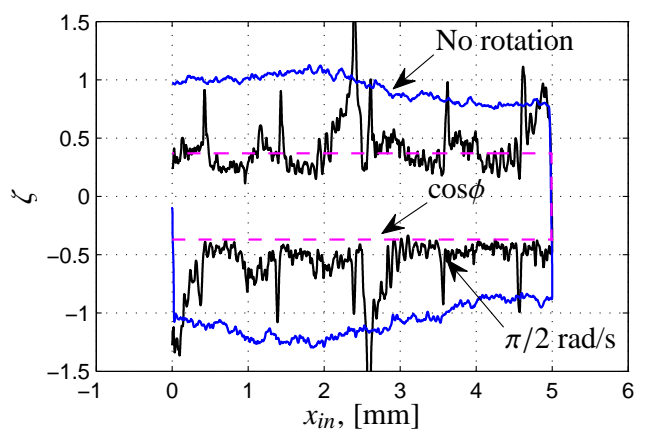

(a) Case 1: $v_{i n}=0.25 \mathrm{~mm} / \mathrm{s}$

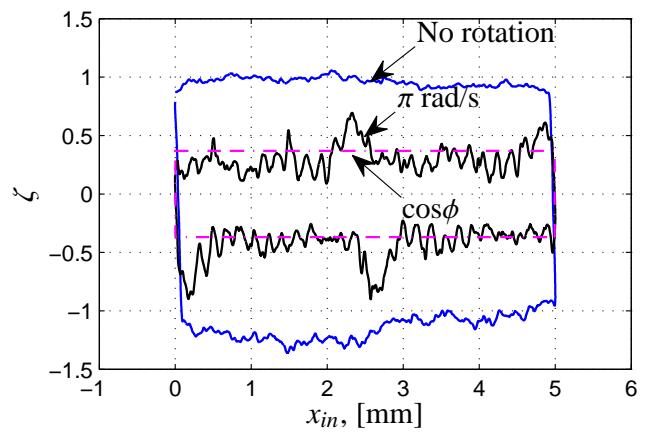

(b) Case 2: $v_{\text {in }}=0.5 \mathrm{~mm} / \mathrm{s}$

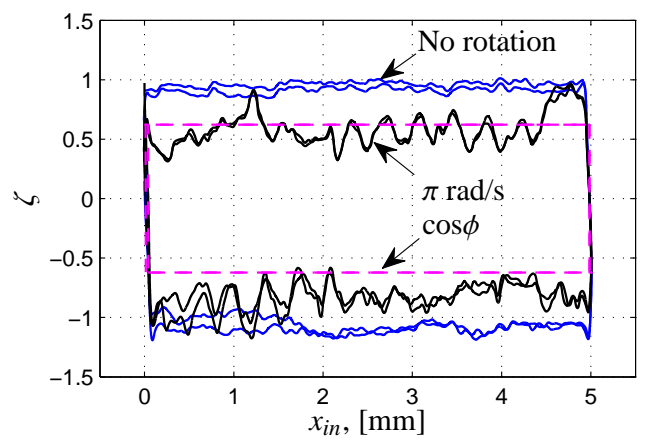

(c) Case 3: $v_{i n}=1.0 \mathrm{~mm} / \mathrm{s}$

Fig. 5.12: Comparison of the ratio $\zeta$ for the combined motion-a rotational speed $\omega_{\text {in }}$ with a translation motion input $v_{i n}$. The corresponding analytical values of the ratio $\zeta=\cos \phi$ for the combined motion are also shown. 
Table 5.3: Comparison of the ratio $\zeta$ for constant $v_{\text {in }}$ combined with constant $\omega_{\text {in }}$

\begin{tabular}{c|c|c|c|c}
\hline & & \multicolumn{3}{|c}{$\zeta$} \\
\hline Translation & Rotation & Analytical & \multicolumn{2}{|c}{ Experimental } \\
\cline { 3 - 5 }$v_{\text {in }},[\mathrm{mm} / \mathrm{s}]$ & $\omega_{\text {in }},[\mathrm{rad} / \mathrm{s}]$ & $\cos \phi$ & mean & $\mathrm{rms}$ \\
\hline \multirow{3}{*}{0.25} & 0 & 1.0 & 1.0 & 0.15 \\
& $\pi / 3$ & 0.51 & 0.71 & 0.24 \\
& $\pi / 2$ & 0.37 & 0.51 & 0.24 \\
\hline \multirow{3}{*}{0.5} & 0 & 1.0 & 1.0 & 0.14 \\
& $\pi / 2$ & 0.62 & 0.48 & 0.13 \\
& $\pi$ & 0.37 & 0.36 & 0.14 \\
\hline \multirow{3}{*}{1.0} & 0 & 1.0 & 1.0 & 0.11 \\
& $\pi / 2$ & 0.85 & 0.95 & 0.20 \\
& $\pi$ & 0.62 & 0.70 & 0.18 \\
\hline
\end{tabular}

The analytical value of the ratio $\zeta$ is also shown for comparison, which is equal to $\cos \phi$.

Again, the combined motion results into a reduction in the value of the $\zeta$. The value of $\zeta$ reduced to 0.36 as compared to the theoretical value of 0.37 for $v_{\text {in }}=0.5 \mathrm{~mm} / \mathrm{s}$ and $\omega_{\text {in }}=\pi \mathrm{rad} / \mathrm{s}$. As the rotation motion corresponding to $\omega_{\text {in }}=\pi \mathrm{rad} / \mathrm{s}$ reverses after $5 \mathrm{~s}$, there is no rotation momentarily. As a result, the value of the ratio $\zeta$ approaches towards 1 at the end of stroke and halfway.

The value of the $\zeta$ is calculated for another value of the angular rotation $\omega_{i n}=\pi / 2 \mathrm{rad} / \mathrm{s}$ combined with the axial motion $v_{i n}$. The results are summarized in Tab. 5.3. The reduction in the ratio $\zeta$ with respect to the velocity ratio is observed. The rms values of $\zeta$ are of the order of 0.15 (see Tab. 5.3).

Case 3: $v_{\text {in }}=1.0 \mathrm{~mm} / \mathrm{s}$ The translation speed $v_{\text {in }}$ is changed to $1.0 \mathrm{~mm} / \mathrm{s}$. The translation motion is combined with different values of rotational speed $\omega_{\text {in }}-0, \pi / 2$ and $\pi \mathrm{rad} / \mathrm{s}$. Both the motions are reversed at the same time after every $5 \mathrm{~s}$. Figure 5.12(c) shows the plot of the ratio $\zeta$ for the rotation input of $\omega_{i n}=\pi \mathrm{rad} / \mathrm{s}$ and translation input of $v_{\text {in }}=1.0 \mathrm{~mm} / \mathrm{s}$. The plot the ratio $\zeta$ is compared for no rotation motion combined with the translation input. We observe the reduction in the value of $\zeta$ as the translation motion is combined with the rotation motion. The analytical value of the ratio $\zeta$ for the given velocity ratio $v_{c} / v_{a}$ is also shown. The value of $\zeta$ reduced to 0.70 as compared to the theoretical value of $\cos \phi=0.62$ for $v_{\text {in }}=1.0 \mathrm{~mm} / \mathrm{s}$ and $\omega_{\text {in }}=\pi \mathrm{rad} / \mathrm{s}$.

The value of the $\zeta$ is calculated for another value of the angular rotation $\omega_{i n}=\pi / 2 \mathrm{rad} / \mathrm{s}$ combined with the axial motion $v_{i n}$. The results are summa- 
rized in Tab. 5.3. The reduction in the ratio $\zeta$ with respect to the velocity ratio is observed. The rms values of $\zeta$ are of the order of 0.20 (see Tab. 5.3).

The translation and rotation inputs are abruptly changing the direction of motion. As a result, we see transient vibrations in both the directions. The velocities in translation and rotation along the wire suffer from oscillations; and they are no more constant as the input motion. The ratio $\zeta$ depends on the velocity ratio at the individual contact points and, therefore, we observe oscillations in the experimental results as shown in Fig. 5.12. This is also reflected in the rms values of $\zeta$ as shown in Tab. 5.3. Nevertheless, the effect of the combined motion on the ratio $\zeta$ is observed in all the cases as hypothesized. The experimental results show that the value of the ratio $\zeta$ decreases as the velocity ratio $v_{c} / v_{a}$ increases as shown in Tab. 5.3.

\section{Constant translation $v_{\text {in }}$ with sinusoidal rotation input}

In this case, the constant velocity input $v_{i n}$ is combined with the sinusoidal rotation input. The sinusoidal rotation input provides a wide range of velocity ratios in one experiment. Moreover, the dynamic effects will be less excited due to a smoother rotation input as compared to the constant rotation input where the direction of rotation changes abruptly.

Case 1: $v_{\text {in }}=0.25 \mathrm{~mm} / \mathrm{s}$ A constant translation input $v_{\text {in }}=0.25 \mathrm{~mm} / \mathrm{s}$ is combined with a sinusoidal rotation input of amplitude $A=2 \pi$ rad and frequency $f=0.1 \mathrm{~Hz}$. The translation motion is reversed after $20 \mathrm{~s}$ and repeated.

The input and output forces are measured and the logarithm of the force ratio, $\ln \left(F_{\text {in }} / F_{\text {out }}\right)$, is calculated. The ratio $\zeta$ is calculated for the entire motion range of $40 \mathrm{~s}$. The analytical value of the ratio $\zeta$ is calculated using Eqn. (5.14) from the input translation motion $v_{i n}$ and the circumferential velocity $v_{c}$ corresponding to the rotation input $\omega_{i n}$. Figure 5.13 shows the plot of the ratio $\zeta$ for the entire motion range. The experimental result showed a similar qualitative behavior as predicted by the analytical solution. There is a large reduction in the value of the ratio $\zeta$ as the rotational speed approaches to the maximum. As the rotational speed approaches towards zero, the value of the ratio $\zeta$ increases to the maximum. The plots corresponding to no rotation motion are also shown for the comparison.

A lag is observed in the response as compared with the analytical value. A lag in the response is expected due to static and dynamic effects. Friction and the stiffness leads to virtual play. The system has finite inertia and limited stiffness, which leads to some lag. However, we try to minimize the dynamic 


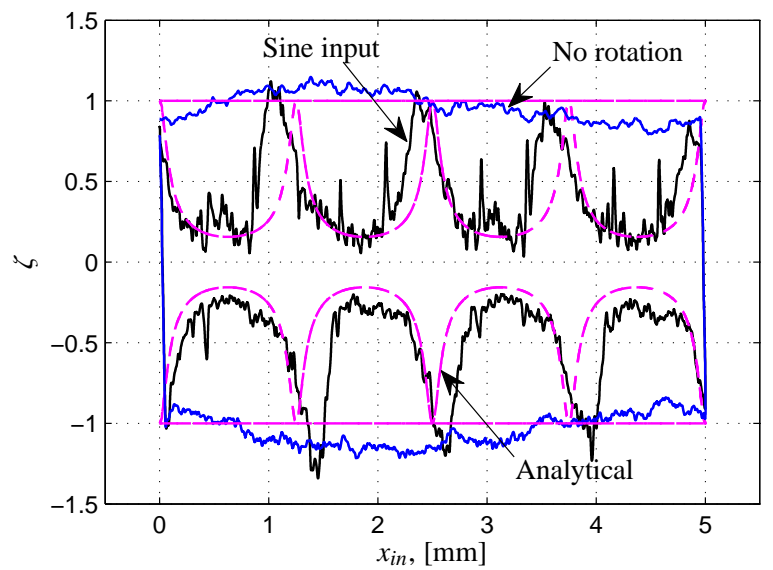

Fig. 5.13: Comparison of the ratio $\zeta$ for the translation motion input $v_{\text {in }}=0.25 \mathrm{~mm} / \mathrm{s}$ combined with the sinusoidal rotational input of amplitude $A=2 \pi$ rad and frequency $f=0.1 \mathrm{~Hz}$. The corresponding analytical value of the ratio $\zeta$ is also shown.

effects by reducing the actuation frequency well below the fundamental frequency (of the order of $2 \mathrm{~Hz}$ ). As we observe from Eqn. (5.14), the ratio $\zeta$ is dependent on the velocity ratio $v_{c} / v_{a}$. The velocities along the length of the instrument lag as compared with the input velocities. Therefore, the instantaneous value of angle $\phi$ and, hence, the analytical value of the ratio $\zeta$ will be different. Since we provided a sinusoidal rotation input and the response depends on the velocity inputs, we applied a Fourier fit of a fundamental frequency of same magnitude as the rotation input. Moreover, we limited the number of harmonics to capture the overall characteristics but not the noise. A Fourier fit is applied to the experimental value of $\zeta$ [37]. The Fourier series is expressed as

$$
\zeta_{f i t}=a_{0}+\sum_{i=1}^{n} a_{i} \cos (n \omega t)+b_{i} \sin (n \omega t) .
$$

Here, the fundamental frequency $\omega=2 \pi f$ and the number of harmonics in the series $n=2$ is used. The fit is performed separately for both forward and reverse stroke of translation motion. The absolute value of the ratio $\zeta$ is analyzed for both the cases. The lag $\delta t$ is obtained by comparing the maximum values of $\zeta$ from the fitted and analytical plots in time. Figure 5.14 shows the fitted curve with the experimental plot of $\zeta$ with respect to the input displacement $x_{i n}$ for both forward and reverse stroke of motion. The analytical plots have been adjusted in time using the obtained lag and are also shown in Fig. 5.14.

The overall reduction in the value of $\zeta$ is obtained from the maximum and 


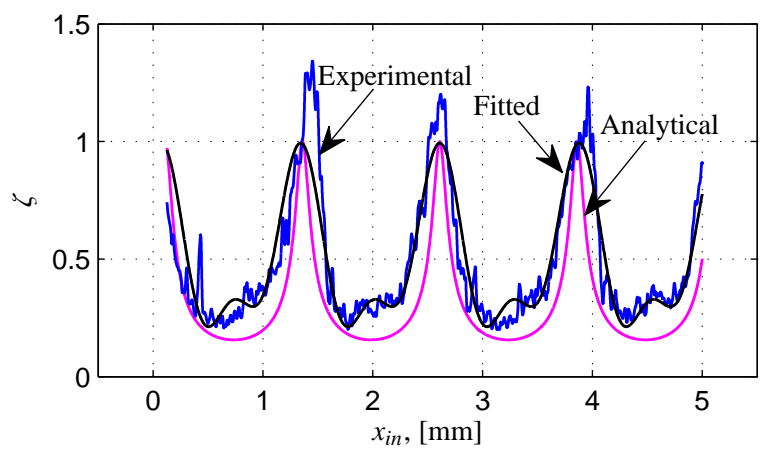

(a) Forward

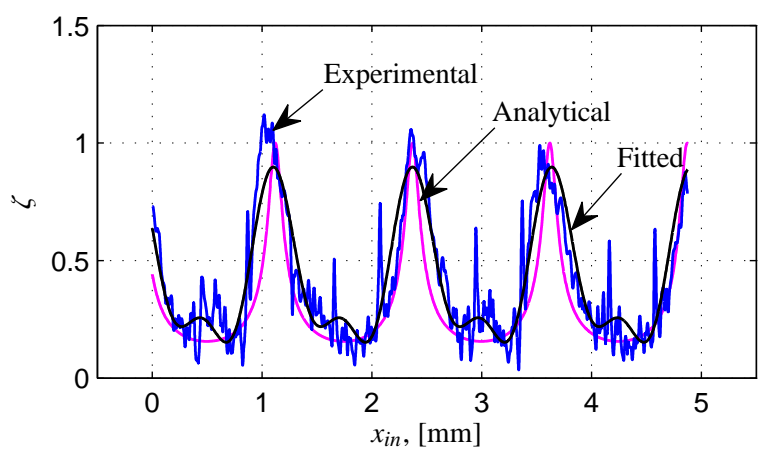

(b) Reverse

Fig. 5.14: Comparison of the ratio $\zeta$ for a translation motion input $v_{i n}=0.25 \mathrm{~mm} / \mathrm{s}$ combined with a sinusoidal rotational input of amplitude $A=2 \pi \mathrm{rad}$ and frequency $f=0.1 \mathrm{~Hz}$. A Fourier fit to the experimental plot and the corresponding shifted analytical plot are also shown. 
minimum values of the fitted curve $\zeta_{f i t}$ and compared with the analytical result. The maximum value corresponds to when there is only translation motion and the minimum value corresponds to the translation motion combined motion with the maximum rotational speed. The results are shown in Tab. 5.4. There is a large reduction in $\zeta$ due to the combined motion. The maximum reduction achieved in this case is $78 \%$ in forward stroke and $75 \%$ in reverse stroke compared to the theoretical reduction of $84 \%$.

The deviation in the ratio $\zeta$ is calculated from the fitted curve as well as from the analytical curve. In this case, the analytical plot has been already shifted according to the lag $\delta t$. The lag is expressed as a non-dimensional parameter $\delta t / T$, where $T=1 / f$. The error in the ratio $\zeta$ is calculated from both the plots. The experimental results deviated by $17 \%$ and $9 \%$ in the forward and reverse stroke of motion as compared to the analytical results. The deviation is due to static and dynamic effects related to friction, inertia and stiffness. The effective frictional force is also not constant as it depends on the instantaneous velocity ratio. Nonetheless, we observed a large reduction in the ratio $\zeta$ if we combine the translation motion with the rotation motion.

Case 2a: $v_{i n}=0.5 \mathrm{~mm} / \mathrm{s} \quad$ Similar experiments are performed with a translation input $v_{\text {in }}=0.5 \mathrm{~mm} / \mathrm{s}$ combined with a sinusoidal rotation input of amplitude $A=2 \pi \mathrm{rad}$ and frequency $f=0.1 \mathrm{~Hz}$. The translation motion is reversed after $10 \mathrm{~s}$. Figure 5.15 shows the plot of the ratio $\zeta$ with respect to the input displacement $x_{i n}$. The experimental and analytical results are compared for the combined motion as well as for no rotation. We observed similar reduction in the value of the ratio $\zeta$ for the combined motion as expected from the analytical result. A Fourier fit with $f=0.1 \mathrm{~Hz}$ and $n=2$ is applied to the experimental data separately for both forward and reverse stroke of translation motion. The measurements were taken for 2 cycles of motion. The lag is obtained by comparing the fitted and analytical plots. The analytical plot is shifted accordingly. Figure 5.16 shows the different plots-experimental, fitted and shifted analytical一for $v_{i n}=0.5 \mathrm{~mm} / \mathrm{s}$ combined with a sinusoidal rotational input of amplitude $A=2 \pi$ rad and frequency $f=0.1 \mathrm{~Hz}$. Different plots are shown only for the forward stroke of motion.

We observed a reduction of $63 \%$ in the value of $\zeta$ in both forward and reverse stroke of motion as compared to $70 \%$ analytically as shown in Tab. 5.4. A mean deviation of $18 \%$ and $9 \%$ is observed in forward and reverse stroke of motion from the analytical solution. A reduction in $\zeta$ is observed for the combined motion in accordance with the analytical result. 


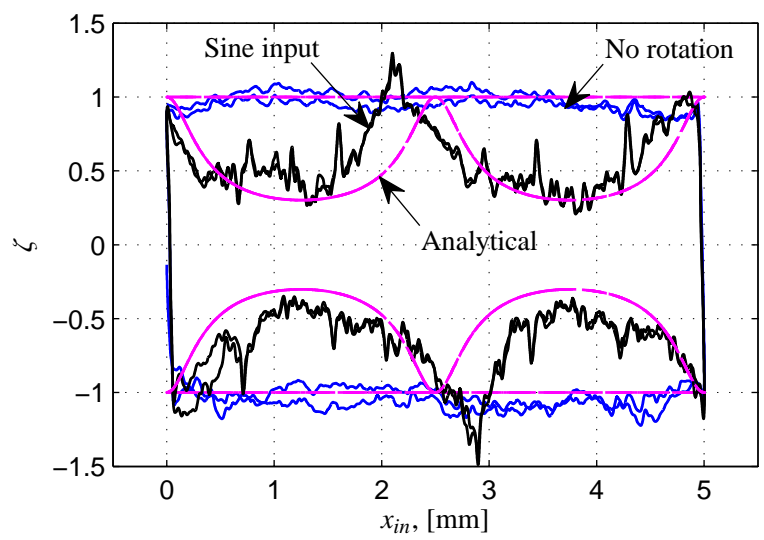

Fig. 5.15: Comparison of the ratio $\zeta$ for the translation motion input $v_{i n}=0.5 \mathrm{~mm} / \mathrm{s}$ combined with the sinusoidal rotational input of amplitude $A=2 \pi$ rad and frequency $f=0.1 \mathrm{~Hz}$. The corresponding analytical value of the ratio $\zeta$ is also shown.

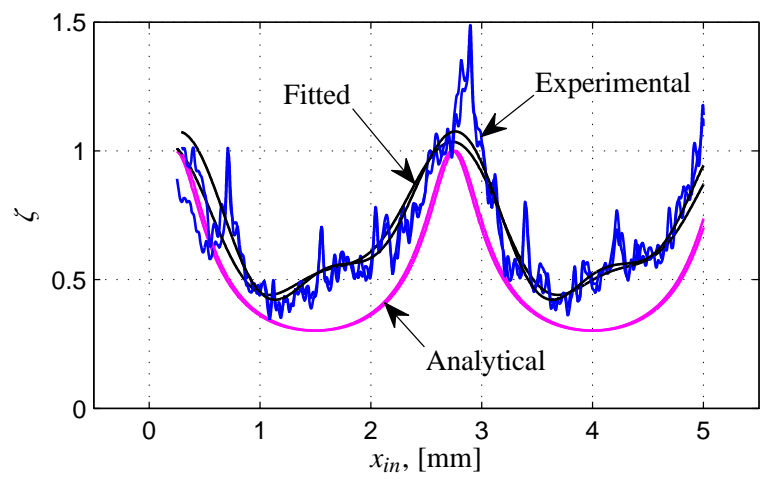

Fig. 5.16: Comparison of the ratio $\zeta$ for a translation motion input $v_{\text {in }}=0.5 \mathrm{~mm} / \mathrm{s}$ combined with a sinusoidal rotational input of amplitude $A=2 \pi \operatorname{rad}$ and frequency $f=0.1 \mathrm{~Hz}$. A Fourier fit to the experimental plot and the corresponding shifted analytical plot are also shown. 
Table 5.4: Value of $\zeta$ for constant $v_{i n}$ combined with sinusoidal rotational input of amplitude $A$ and frequency $f$

\begin{tabular}{|c|c|c|c|c|c|c|c|c|c|}
\hline \multirow{3}{*}{$\begin{array}{c}v_{\text {in }} \\
(\mathrm{mm} / \mathrm{s})\end{array}$} & \multirow{3}{*}{$\begin{array}{c}A \\
(\mathrm{rad})\end{array}$} & \multirow{3}{*}{$\begin{array}{c}f \\
(\mathrm{~Hz})\end{array}$} & \multirow{3}{*}{ Direction } & \multicolumn{6}{|c|}{ Value of $\zeta$} \\
\hline & & & & \multicolumn{2}{|c|}{ Analytical } & \multicolumn{4}{|c|}{ Experimental } \\
\hline & & & & $\min$ & $\Delta \zeta$ & $\max$ & $\min$ & $\Delta \zeta$ & $\overline{\delta t / T}$ \\
\hline \multirow{2}{*}{0.25} & \multirow{2}{*}{$2 \pi$} & \multirow{2}{*}{0.1} & Forward & 0.16 & 0.84 & 0.99 & 0.21 & 0.78 & -0.04 \\
\hline & & & Reve & 0.16 & 0.84 & 0.90 & 0.15 & 0.75 & -0.05 \\
\hline \multirow{8}{*}{0.50} & \multirow{4}{*}{$2 \pi$} & \multirow{4}{*}{0.1} & orward & 0.30 & 0.70 & 1.04 & 0.44 & 0.60 & -0.05 \\
\hline & & & & & & 1.08 & 0.42 & 0.66 & -0.05 \\
\hline & & & Reve & 0.30 & 0.70 & 0.99 & 0.35 & 0.64 & -0.06 \\
\hline & & & & & & 0.98 & 0.36 & 0.62 & -0.06 \\
\hline & \multirow{4}{*}{$3 \pi$} & \multirow{4}{*}{0.2} & Fo & 0.11 & 089 & 1.14 & 0.19 & 0.95 & -0.04 \\
\hline & & & & & & 1.14 & 0.20 & 0.94 & -0.04 \\
\hline & & & & 011 & 080 & 0.99 & 0.15 & 0.84 & -0.05 \\
\hline & & & & 0.11 & & 0.99 & 0.14 & 0.85 & -0.05 \\
\hline \multirow{4}{*}{1.0} & \multirow{4}{*}{$3 \pi$} & \multirow{4}{*}{0.2} & & 021 & 07 & 1.03 & 0.32 & $\mid 0.71$ & -0.03 \\
\hline & & & u & 0.21 & 0.19 & 1.09 & 0.33 & 0.76 & -0.03 \\
\hline & & & e & 021 & 079 & 0.94 & 0.26 & 0.68 & -0.05 \\
\hline & & & & & & 0.94 & 0.27 & 0.67 & -0.0 \\
\hline
\end{tabular}




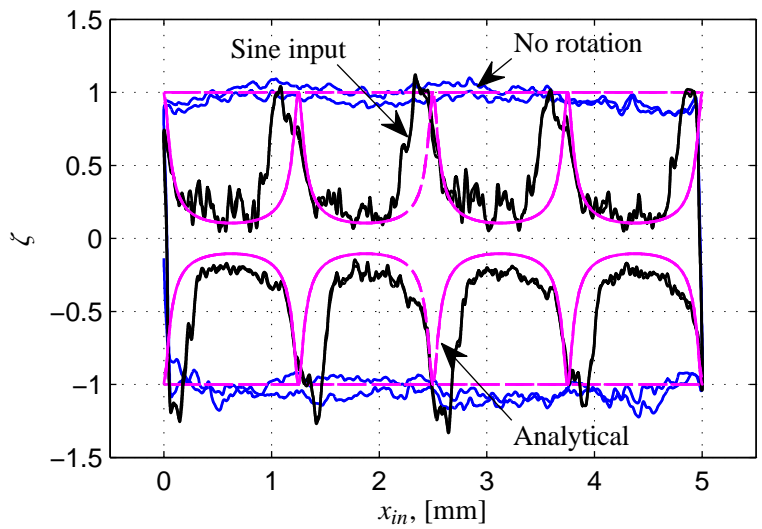

Fig. 5.17: Comparison of the ratio $\zeta$ for the translation motion input $v_{i n}=0.5 \mathrm{~mm} / \mathrm{s}$ combined with the sinusoidal rotational input of amplitude $A=3 \pi \mathrm{rad}$ and frequency $f=0.2 \mathrm{~Hz}$. The corresponding analytical value of the ratio $\zeta$ is also shown.

Case 2b: $v_{\text {in }}=0.5 \mathbf{~ m m} / \mathbf{s}$ In this case, we combined the translation input $v_{\text {in }}=0.5 \mathrm{~mm} / \mathrm{s}$ with a sinusoidal rotation input of amplitude $A=3 \pi \mathrm{rad}$ and frequency $f=0.2 \mathrm{~Hz}$. The amplitude and frequency of the rotation input are increased to get a higher velocity ratio $v_{c} / v_{a}$ and, thus, to achieve a higher reduction in $\zeta$. Figure 5.17 shows the plot of the ratio $\zeta$ with respect to the input displacement $x_{i n}$. We observed a further reduction in the value of the ratio $\zeta$ as compared to Fig. 5.15. Here, a Fourier fit with $f=0.2 \mathrm{~Hz}$ and $n=3$ is applied to the experimental value of $\zeta$. The analytical plot is shifted by the lag. The results are compared separately for the forward and reverse stroke of translation input for 2 cycles. Figure 5.18 shows the different plots of the ratio $\zeta$ for the forward stroke of motion.

We obtained an increased reduction of $95 \%$ and $85 \%$ in $\zeta$ in the forward and reverse stroke of tranlation input as compared to analytical reduction of $89 \%$ as shown in Tab. 5.4. We observed a mean deviation of $22 \%$ and $14 \%$ in $\zeta$ in the forward and reverse stroke of motion as compared to the analytical solution. The deviation is larger as compared to the previous case. However, the overall reduction in $\zeta$ is observed for both forward and reverse stroke of motion in accordance with the analytical solution.

Case 3: $v_{\text {in }}=1.0 \mathrm{~mm} / \mathrm{s}$ In this case, the translation input motion is increased to $v_{\text {in }}=1.0 \mathrm{~mm} / \mathrm{s}$. The direction of motion changes after $5 \mathrm{~s}$. The rotation input is given as a sinusoidal input of amplitude $A=3 \pi$ rad and frequency $f=0.2 \mathrm{~Hz}$. Figure 5.19 shows the plot of the ratio $\zeta$ with respect to 


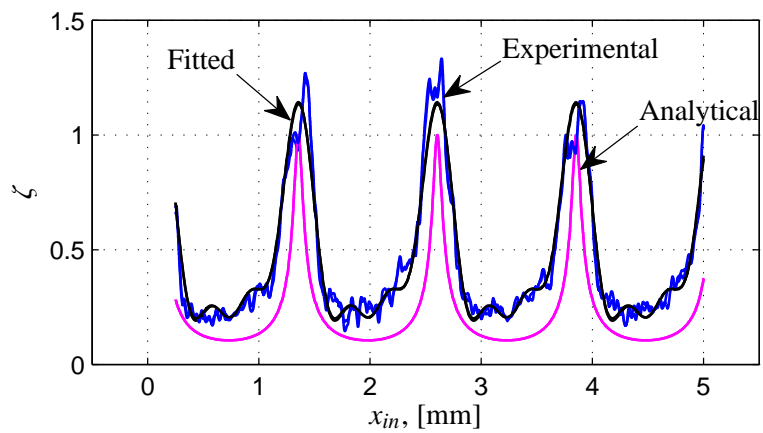

Fig. 5.18: Comparison of the ratio $\zeta$ for a translation motion input $v_{i n}=0.5 \mathrm{~mm} / \mathrm{s} \mathrm{combined}$ with a sinusoidal rotational input of amplitude $A=3 \pi$ rad and frequency $f=0.2 \mathrm{~Hz}$. A Fourier fit to the experimental plot and the shifted analytical plot are also shown.

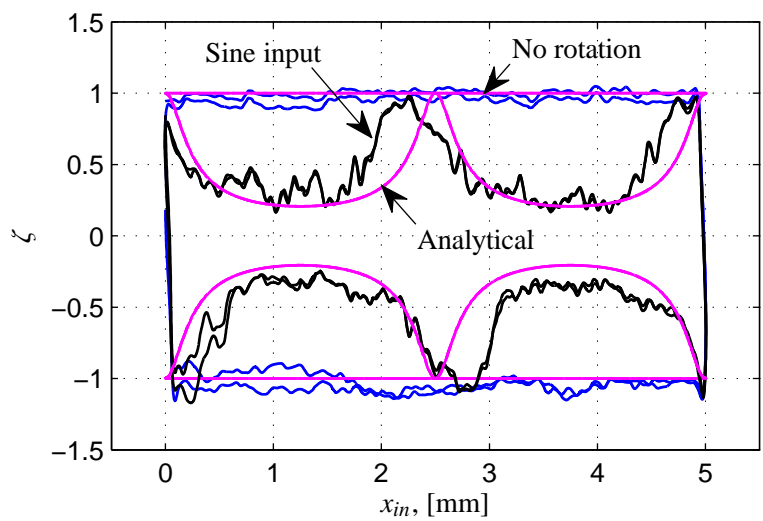

Fig. 5.19: Comparison of the ratio $\zeta$ for the translation motion input $v_{i n}=1.0 \mathrm{~mm} / \mathrm{s} \mathrm{combined}$ with the sinusoidal rotational input of amplitude $A=3 \pi \mathrm{rad}$ and frequency $f=0.2 \mathrm{~Hz}$. The corresponding analytical value of the ratio $\zeta$ is also shown.

the input displacement $x_{i n}$. The experimental results show a reduction in $\zeta$ as expected from the analytical results. A lag is also observed as in the previous cases. A Fourier fit with $f=0.2 \mathrm{~Hz}$ and $n=3$ is applied separately for the forward and reverse stroke of translation input for 2 cycles. Figure 5.20 shows the experimental, fitted and the analytical shifted plots for $\zeta$ for the forward stroke of motion.

We obtained a reduction of $73 \%$ and $67 \%$ in $\zeta$ for the forward and reverse stroke of translation motion as compared to analytical reduction of $79 \%$ as shown in Tab. 5.4. A mean deviation of $16 \%$ and $7 \%$ is observed in the forward and reverse stroke of motion as compared with the analytical solution. 


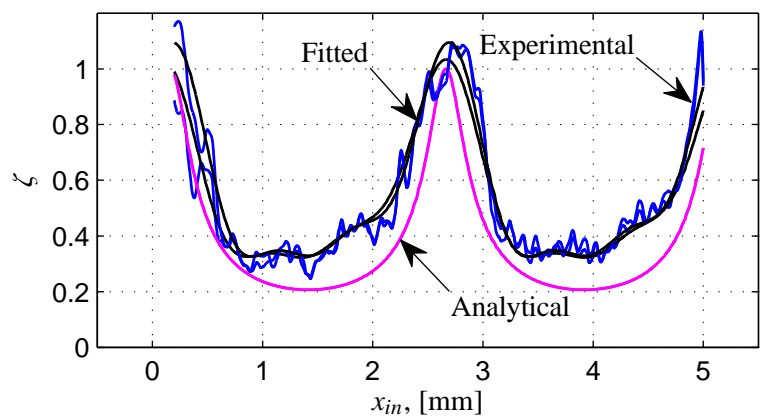

Fig. 5.20: Comparison of the ratio $\zeta$ for a translation motion input $v_{i n}=1.0 \mathrm{~mm} / \mathrm{s}$ combined with a sinusoidal rotational input of amplitude $A=3 \pi \mathrm{rad}$ and frequency $f=0.2 \mathrm{~Hz}$. A Fourier fit to the experimental plot and the corresponding analytical plot are also shown.

\subsection{Discussion}

Assumptions are made to simplify the analytical model and use it as a tool to estimate the improvement in the force transmission due to the combined motion. We assumed uniform contact and motion transmission along the length of the flexible instrument. However, as the wire is flexible in bending and rotation, the motion at the distal end and all the intermediate contact points will not be same as the input motion at the proximal end. In this study, we compared the simulation and experimental results with the analytical result with respect to the input motion both in translation and rotation. The analytical formulation assumes that the wire is fully compliant in bending and rigid in axial direction and torsion. Nonetheless, the analytical formulation provides the basis of the study and can be used as a guiding principle for further study.

Friction, inertia and the compliance of the instrument leads to deviation from the analytical formulation. The effect of these parameters are observed in the experiments. The flexible multibody can be useful to further investigate the effect of combined motion on various kinematic and dynamic behaviour.

Friction between the instrument and the tube is another challenge. The value of $\mu$ is not constant and varies over a range. Therefore, we estimated the value of $\mu$ for each set of experiments. Nonetheless, we are keen on overall reduction in $\zeta$ over a cycle, the absolute value of the friction force is not required.

The reduction in $\zeta$ leads to improved force transmission along the axial direction. The application is not only limited to the robotic surgical application, but can be also applied to many mechanical transmission problems using flexible wires through tubes. 


\subsection{Conclusion}

In this paper, we presented how the force transmission of a flexible instrument inside a tube is improved along the axial direction by combining the translation motion input with rotational motion input. An analytical model is derived to calculate the reduction in friction force. The ratio $\zeta$ is defined to measure the reduction in the friction force along the axial direction due to the combined motion. We set up a flexible multibody model of an instrument inside a curved rigid tube and compared the simulation results with the analytical solution. The simulation result showed a reduction of $80 \%$ in the value of $\zeta$ in accordance with the analytical result for the given velocity ratio $v_{c} / v_{a}$.

We showed the reduction in the ratio $\zeta$ through several experiments combining different constant translation input $v_{\text {in }}$ with constant and sinusoidal rotation input with different amplitudes and frequencies. A maximum reduction of $84 \%$ is obtained against a reduction of $89 \%$ calculated analytically. Static and dynamic effects lead to a deviation from the analytical solution. The reduction in the friction force ultimately results into improved force transmission. The developed analytical model offers a simple though effective estimate of the amount of reduction in friction force in axial direction. The knowledge of force transmission with a combination of motions can be used to increase the force fidelity of a flexible instrument in applications like robotic surgery with a flexible instrument. The developed insight can also be useful in designing mechanical transmission using flexible wires, cables and tubes.

\section{Acknowledgment}

This research is funded with the TeleFLEX project, number PID07038, by the Dutch Department of Economic Affairs, Agriculture and Innovation and the Province of Overijssel, within the Pieken in de Delta (PIDON) initiative. 



\section{Conclusions AND RECOMMENDATIONS}

\subsection{Conclusions}

The overall objectives of the research are restated as:

Characterization of static and dynamic behaviour of a flexible surgical instrument inside a curved rigid tube-both for translation and rotation motion input; develop a 2-D and 3-D flexible multibody model; design a dedicated experimental set-up for the actuation and measurement of motion and interaction forces; and to derive strategies to improve motion and force fidelity of the instrument.

The objectives and the conclusions of the individual chapters are recapitulated in the following sections. The overall conclusion is discussed thereafter in Section 6.1.5. The recommendations for the future work are summarized in Section 6.2. 


\subsubsection{2-D Flexible Multibody Modelling}

The objective of Chapter 2 is:

Develop a 2-D flexible multibody model of an instrument to study its sliding behaviour inside a curved rigid tube.

The focus is to develop a computational model for a planar case which incorporates not only the geometry but also the necessary mechanical properties of the system and the contact model. The model should be able to determine the characteristic behaviour during insertion and for fine manipulation of the instrument in translation.

A 2-D flexible multibody model of a flexible instrument constrained inside a curved rigid tube was developed. A static friction based contact model was implemented. The simulations showed the forces acting at different nodes on the instrument during insertion inside a curved tube of different shapes. The simulation results for fine manipulation showed motion hysteresis and stickslip behaviour. The coefficient of friction has a large influence on the motion hysteresis whereas the bending rigidity of the instrument has little influence.

\subsubsection{Design and Evaluation of an Experimental Set-up}

The objective of Chapter 3 is:

Design and evaluation of an experimental set-up for the validation of the developed flexible multibody model and characterization of flexible instruments for surgical application.

The design specifications were chosen with respect to a typical surgical scenario — with a flexible instrument inside an endoscope-where the surgeon controls the instrument from its proximal end. The experimental set-up consists of three basic modules-Actuation module (AM), Force sensing module (FSM), and Tip motion measurement module (T3M).

The 2-DOFs AM provides the actuation in axial and twist direction from the proximal end of the flexible instrument independently with a resolution of $0.1 \mu \mathrm{m}$ and $0.005 \mathrm{deg}$ respectively. The 2-DOFs T3M achieves contactless measurement of the distal end motion in axial and twist direction via an air bearing guided cam. A measurement resolution of $15 \mu \mathrm{m}$ and $0.15 \mathrm{deg}$ are achieved in translation and rotation. The 6-DOFs FSM measures the force and moment exerted by the flexible instrument on the curved tube. The force resolution of $9 \mathrm{mN}$ and the force and moment error of $0.8 \%$ and $2.5 \%$ respectively are achieved by the FSM. The three modules of the set-up conform to 
the design objectives and hence were suited for the validation of the developed model of the flexible instrument inside a curved rigid tube.

\subsubsection{3-D Modelling and Experimental Validation}

The objective of Chapter 4 is:

Set up a 3-D flexible multibody model of a surgical instrument to study its translational and rotational behavior in a 3-D environment, and validate the model with experimental results.

A 3-D flexible multibody model of the instrument was set up. The 2-D model was able to characterize the instrument behaviour only in translation and for only a planar tube (Chapter 2). The developed 3-D model is able to characterize the instrument behaviour in both translation and rotation, and for any 3-D tube geometry. The contact model is redefined with respect to 3-D tubes of different geometry. The effect of various parameters in motion and force transmission was studied through various simulations in translation and rotation.

The simulations show the stick-slip behavior and motion hysteresis when the input velocity changes direction. However, the magnitude of stick-slip is greatly reduced when the distal end is preloaded in tension. The motion hysteresis is reduced from $2.8 \mathrm{~mm}$ to $0.6 \mathrm{~mm}$. The ratio of the input force at the proximal end to the spring load at the distal end is compared with the capstan equation. The simulation results show a deviation of $1.8 \%$ in the estimation of input force. The simulations for rotational motion show a motion hysteresis of the order of 0.4 degree for the unloaded case. The motion hysteresis in rotation increases considerably with different flexural rigidity along the two principal axes and with a pre-curvature. A difference in the flexural rigidity along the principal axes and a pre-curvature result in a whipping action.

Experiments are performed to validate the developed model for motion and force transmission in translation and rotation using the designed experimental set-up (Chapter 3). The set-up is also used to estimate the coefficient of friction $\mu$. Measurement of the input force shows a deviation of the order of $1 \%$ from the analytical solution obtained from the capstan equation. The measured output rotation of the distal end with respect to the input rotation of the proximal end is in agreement with the simulation result. The developed 3-D model of a flexible instrument in a curved rigid tube is used to study the effect of various parameters for different geometric configurations for both in translation and rotation. 


\subsubsection{Improved Force Transmission}

The objective of Chapter 5 is:

Improve the force transmission of the flexible instrument along the axial direction through a curved rigid tube by combining the translation input motion with rotational motion.

An analytical model is derived to calculate the reduction in friction force and, thus, the improvement in the force transmission along the axial direction by combining the translation motion input with a rotational motion input. The ratio $\zeta$ is defined to measure the reduction in the friction force along the axial direction due to the combined motion. A flexible multibody model of an instrument inside a curved rigid tube was set up and the simulation results are compared with the analytical solution. The simulation result showed a reduction of $80 \%$ in the value of $\zeta$ in accordance with the analytical result for the given velocity ratio $v_{c} / v_{a}$.

Several experiments were performed with different combinations of constant translation input $v_{\text {in }}$ with constant and sinusoidal rotation input with different amplitudes and frequencies. A reduction of $84 \%$ is obtained against a reduction of $89 \%$ calculated analytically. Static and dynamic effects lead to a deviation from the analytical solution. The reduction in the friction force ultimately results into improved force transmission. The developed analytical model offers a simple though effective estimate of the amount of reduction in friction force in axial direction. The knowledge of force transmission with a combination of motions can be used to increase the force fidelity of a flexible instrument in applications like robotic surgery with a flexible instrument. The developed insight can also be useful in designing mechanical transmission using flexible wires, cables and tubes.

\subsubsection{Overall Conclusion}

In this thesis, we highlighted the increased use of a flexible endoscope and instruments to perform advanced surgical procedures. We characterized the static and dynamic behaviour of a flexible instrument using developed computational models and experiments. We developed a 2-D and 3-D flexible multibody model of a flexible instrument inside a curved rigid tube (Chapter 2 and Chapter 4 respectively). We studied the motion and force transmission characteristics of a flexible wire in a curved rigid tube using the 2-D and 3-D model. For a planar case, the 2-D model can be used to characterize the instrument behaviour in any complex-shaped tube in translation. However, the 3-D model 
will be needed for the rotational behaviour as well as for a complex-shaped tube in 3-D space. We showed the effect of friction and bending stiffness on motion hysteresis (Chapter 2). We also showed the effect of different flexural rigidities $\left(E I_{y} \neq E I_{z}\right)$ along the principal axes of the wire and of the pre-curvature on the whipping action which is highly undesired and unsafe for surgical application (Chapter 4). The force transmission characteristics were compared with the capstan equation and with the experimental results. We designed an experimental set-up and evaluated with respect to the design specifications required for model validation and characterization of the instrument (Chapter 3). The set-up enabled us to measure all the six components of the interaction force between the instrument and the curved rigid tube. Different combinations of motion in translation and rotation can be given to the proximal end of the wire and the output motion of the distal end can be measured simultaneously. We used the set-up and performed several experiments with different tubes and different dimensions of flexible wires. This led us to the research of combined input motions to improve force transmission. We developed the analytical model; and used the developed 3-D model and the experimental set-up to validate the analytical result. The force transmission of a flexible instrument has improved considerably by combining the translation with the rotational input motion (Chapter 5).

Therefore, we can conclude that we achieved the objectives of the research stated in the beginning of this thesis. We can use the developed model to predict the motion and force transmission characteristics of a flexible instrument for the given mechanical properties of the instrument, contact parameters and the geometry of the endoscope. Inversely, we can also calculate or recommend the desired mechanical properties of the flexible instrument for given motion and force transmission characteristics of the instrument. The developed strategy to alleviate frictional force and, thus, to increase force transmission, can be implemented in the overall control structure to bring more transparency into the system. The set-up can be used to measure the motion and forces at the proximal and distal ends of the instrument to further characterize the instrument behaviour. A better insight into a flexible instrument behaviour inside an endoscope leads to its successful and safe introduction in a master-slave robotic system. This also brings more confidence in using flexible devices for clinical usage. 


\subsection{Recommendations}

We highlighted some pertinent questions towards introducing flexible endoscopic systems for advanced surgical procedures in Chapter 1. The future research directions are summarized with respect to limitations with computational modelling, characterization of actual or developed instruments for advanced surgical procedures and improvement in the experimental set-up in the following sections.

\subsubsection{Modelling and Simulation}

A good computational model has to be accurate and fast. There are two key areas where the future research can focus towards achieving an accurate and comprehensive model for flexible endoscopic surgical system and its interaction with the tissues.

- Flexible endoscope and interaction with tissues. A computational model is required to develop insight how a flexible endoscope interacts with the surrounding organs and how the motion of the endoscope shaftinsertion and twist - facilitates proper navigation and avoids looping inside the body. Moreover, the interaction of the instrument tip with the tissues will also exert forces on the endoscope and can deform the overall geometry. An advanced model should be able to include all these features and can be used to develop more insight in handling and carrying out surgical interventions.

- Computational time. The simulations for rotational input were computationally very heavy and were taking hours and even days in some cases. Friction is one of the problems, particularly, near the zero motion range. Ways to reduce the overall computational time are necessary without affecting the accuracy. This will be desired as we want to use the model to predict the behaviour under more complex scenarios like when the endoscope is fully inside the colon. The computational time will be critical when all the components of the model are there and we want to simulate the individual behaviour.

\subsubsection{Design of a Flexible Instrument}

The instrument has a very critical role in accomplishing a surgical task successfully and safely. It has to be flexible but, at the same time, it has to be rigid enough to transmit the motion and force required to accomplish the task at the 
distal end. The advanced surgical procedures entail new design requirements for the instrument. It is also equally important to know how these mechanical properties affect the overall performance. Integrating these instruments with the robotic technology poses new requirements. Therefore, future directions can be proposed with respect to the design and integration of flexible instruments for advanced surgical procedures as follows:

- Measurement of mechanical properties of an instrument. The model of the instrument requires mechanical properties of the actual instrument. An average and equivalent mechanical property can be obtained through various experiments. An FEM model can be also investigated incorporating all the construction details of the instrument. The same can be applied to the flexible endoscope. Estimation of physical parameters like friction and damping is also very challenging, but they are necessary components of the modelling to get sufficiently good result.

- Develop a building block to use in system model. A system block can be identified consisting of a curved tube in one direction with radius of curvature and angle of wrap. A known characteristic behaviour of a flexible instrument inside a curved tube consisting of one bend leads to define parameters of a transfer block in system definition. The entire complex shape of the tube can be broken down into several curved tubes of single bends and the system behaviour can be analyzed. This can be useful in model-based control design.

\subsubsection{Improvement in the Experimental set-up}

The developed experimental set-up fulfills the requirement necessary for the model validation. However, the design of the set-up can be further improved to include accurate actuation and measurement. The following recommendations are made to improve the experimental set-up for future applications:

- The AM used geared motors for actuation in translation and rotation. This introduces backlash. Moreover, due to a high gear ratio, the motor is able to overcome any fluctuation in the load. However, it is not desired in a real surgical application as the motor does not see any change in the load and can be dangerous. Therefore, a direct drive is preferred.

- The FSM can be re-calibrated using proper calibration techniques to improve the accuracy further. 
- The cam used in the T3M has large rotational inertia together with the shaft used for the attachment. Due to the limited rotational stiffness of the slender wire, the natural frequency is of the order of $1-2 \mathrm{~Hz}$, depending on the diameter and length of the wire. The cam and the attachment can be redesigned to reduce the inertia.

\subsubsection{Other Applications}

In medical science, the developed model can be used for catheter insertion. The model can be further developed to include catheter interaction with the blood and artery walls.

Cable-driven system can be analyzed using the developed model. Many systems, these days, employ cable or wire transmission to actuate end manipulator from the base. Flexible or rigid tubes are used to guide these cables. The motion and force transmission of the cables are greatly reduced due to friction and clearance.

There are industrial endoscopic systems also which are used for inspection and maintenance particularly in the aerospace industry. New tools can be developed to access and maintain things in the complex and hard to reach spaces. 


\section{REFERENCES}

[1] R. G. K. M. Aarts and J. B. Jonker. Dynamic simulation of planar flexible link manipulators using adaptive modal integration. Multibody System Dynamics, 7(1):31-50, 2002.

[2] R. G. K. M. Aarts, J. P. Meijaard, and J. B. Jonker. Flexible multibody modelling for exact constraint design of compliant mechanisms. Multibody System Dynamics, 27(1):119-133, 2012.

[3] R. A. Beasley and R. D. Howe. Increasing accuracy in image-guided robotic surgery through tip tracking and model-based flexion correction. Robotics, IEEE Transactions on, 25(2):292-302, 2009.

[4] G. Berci and K. A. Forde. History of endoscopy. Surgical Endoscopy, 14(1):5-15, 2000.

[5] D. L. Blanding. Exact Constraint: Machine Design Using Kinematic Principles. ASME Press, New York, 1999.

[6] D. B. Camarillo, T. M. Krummel, and J. K. Salisbury. Robotic technology in surgery: Past, present, and future. The American Journal of Surgery, 188(Supplement 1):2-15, 2004.

[7] R. Chamberlain and S. Sakpal. A Comprehensive Review of SingleIncision Laparoscopic Surgery (SILS) and Natural Orifice Transluminal Endoscopic Surgery (NOTES) Techniques for Cholecystectomy. Journal of Gastrointestinal Surgery, 13(9):1733-1740, Sept. 2009.

[8] J. Dankelman, C. A. Grimbergen, and H. G. Stassen. Engineering for Patient Safety: Issues in Minimally Invasive Procedures. LEA's Human Error and Safety Series. Lawrence Erlbaum Associates, Inc., Mahwah, New Jersey 07430, 2005. 
[9] B. R. Dewey. Computer Graphics for Engineers. Harper \& Row, Publishers, New York, 1988.

[10] DuPont Corporation. KEVLAR ${ }^{\circledR}$ technical guide. http://www2 . dupont. com, May 2011.

[11] J. D. Foley, A. van Dam, S. K. Feiner, and J. F. Hughes. Computer Graphics: Principles and Practice. Addison-Wesley Publishing Company, Inc., Reading, Massachusetts, 2nd edition, 1990.

[12] M. Geradin and A. Cardona. Kinematics and dynamics of rigid and flexible mechanisms using finite elements and quaternion algebra. Сотрutational Mechanics, 4(2):115-135, 1988.

[13] J. M. Gere and S. P. Timoshenko. Mechanics of MATERIALS. Stanley Thornes (Publishers) Ltd, United Kingdom, 4th SI edition, 1999.

[14] P. Gomes. Surgical robotics: Reviewing the past, analysing the present, imagining the future. Robotics and Computer-Integrated Manufacturing, 27(2):261-266, 2011. Translational Research - Where Engineering Meets Medicine.

[15] W. B. J. Hakvoort, J. P. Meijaard, R. G. K. M. Aarts, J. B. Jonker, and J. M. Zwikker. Modeling a coriolis mass flow meter for shape optimization. In A. Mikkola and W. Schiehlen, editors, The $1^{\text {st }}$ Joint International Conference on Multibody System Dynamics, pages 1-10, Lappeenranta, Finland, May 25-27 2010. Lappeenranta University of Technology.

[16] C. G. Heyd. The evolution of modern surgery. The American Journal of Surgery, 51(1):54-65, 1941.

[17] Honeywell Sensing \& Control, Columbus, OH 43228. Miniature Load Cell, 2010.

[18] HUMUSOFT ${ }^{\circledR}$, Czech Republic. MF624, Multifunction I/O Card, User's Manual, 2010.

[19] J. D. Jansen. Nonlinear Dynamics of Oilwell Drillstrings. $\mathrm{PhD}$ thesis, Technische Universiteit Delft, the Netherlands, 1993.

[20] B. Jonker. A Finite Element Dynamic Analysis of Flexible Spatial Mechanisms and Manipulators. PhD thesis, Delft University of Technology, Delft, The Netherlands, 1988. 
[21] J. B. Jonker and J. P. Meijaard. Multibody Systems Handbook, chapter SPACAR - computer program for dynamic analysis of flexible spatial mechanisms and manipulators, pages 123-143. Springer-Verlag, Berlin, 1990.

[22] J. B. Jonker and J. P. Meijaard. Deformation modes and dual stress resultants of spatial beam elements in large deflection multibody system analyses. In Proceedings of the $2^{\text {nd }}$ Joint International Conference on Multibody System Dynamics, pages 1-10, Stuttgart, Germany, May, 19June, 12012.

[23] J. H. Jung, N. Pan, and T. J. Kang. Capstan equation including bending rigidity and non-linear frictional behavior. Mechanism and Machine Theory, 43(6):661 - 675, 2008.

[24] V. Karimyan, M. Sodergren, J. Clark, G.-Z. Yang, and A. Darzi. Navigation systems and platforms in natural orifice translumenal endoscopic surgery (notes). International Journal of Surgery, 7(4):297 - 304, 2009.

[25] J. P. Khatait. Motion and Force Transmission of a Flexible Instrument Inside a Curved Endoscope. PhD thesis, University of Twente, Netherlands, 2013.

[26] J. P. Khatait, D. M. Brouwer, R. G. K. M. Aarts, and J. L. Herder. Modeling of a flexible instrument to study its sliding behavior inside a curved endoscope. Journal of Computational and Nonlinear Dynamics, 8(3):(10 pages), 2012. 031002 (Oct 30, 2012).

[27] J. P. Khatait, D. M. Brouwer, R. G. K. M. Aarts, and J. L. Herder. Test set-up to study the behavior of a flexible instrument in a bent tube. In the ASME 2012 Design of Medical Devices Conference, DMD2012, Minneapolis, Minnesota, USA, April 10-12 2012. American Society of Mechanical Engineers, New York.

[28] J. P. Khatait, D. M. Brouwer, J. P. Meijaard, R. G. K. M. Aarts, and J. L. Herder. 3-D multibody modeling of a flexible surgical instrument inside an endoscope. In the ASME 2012 International Mechanical Engineering Congress $\mathcal{E}$ Exposition, IMECE2012, Houston, Texas, USA, November 9-15 2012. American Society of Mechanical Engineers, New York.

[29] J. P. Khatait, D. M. Brouwer, J. P. Meijaard, R. G. K. M. Aarts, and J. L. Herder. Flexible multibody modeling of a surgical instrument inside an 
endoscope. Journal of Computational and Nonlinear Dynamics, 2013. draft submitted, under review.

[30] J. P. Khatait, D. M. Brouwer, H. M. J. R. Soemers, R. G. K. M. Aarts, and J. L. Herder. Design of an experimental set-up to study the behavior of a flexible surgical instrument inside an endoscope. Journal of Medical Devices, 7(3):(12 pages), 2013. 031004(Jul 03, 2013).

[31] J. P. Khatait, M. Krijnen, J. P. Meijaard, R. G. K. M. Aarts, D. M. Brouwer, and J. L. Herder. Modelling and simulation of a flexible endoscopic surgical instrument in a tube. ASME Conference Proceedings, 2011(54884):557-566, 2011.

[32] A. R. Lanfranco, A. E. Castellanos, J. P. Desai, and W. C. Meyers. Robotic surgery: a current perspective. Annals of surgery, 239(1):14 21, January 2004.

[33] Laparoscopic Surgery. Laparoscopy in gynecology. http://wwW . laparoscopy. am, April 2013.

[34] M. J. Mack. Minimally invasive and robotic surgery. JAMA: the journal of the American Medical Association, 285(5):568-572, February 2001.

[35] The MathWorks, Inc., 3 Apple Hill Drive, Natick, MA 01760-2098. Signal Processing Toolbox ${ }^{T M}$ Users Guide, 2010.

[36] The MathWorks, Inc., 3 Apple Hill Drive, Natick, MA 01760-2098. xPC Target $^{T M}$ Users Guide, 2010.

[37] The MathWorks, Inc., 3 Apple Hill Drive, Natick, MA 01760-2098. Curve Fitting Toolbox ${ }^{T M}$ Users Guide, 2013.

[38] Maxon Motor Ag, 6072 Sachseln, Switzerland. High Precision Drives and Systems, 09/10 edition, 2010.

[39] Mayo Foundation for Medical Education and Research (MFMER). Upper endoscopy. http://www.mayoclinic.com/health/medical/ IM04428, May 2013.

[40] J. P. Meijaard. Validation of flexible beam elements in dynamics programs. Nonlinear Dynamics, 9:21-36, 1996. 
[41] J. P. Meijaard. Application of Runge-Kutta-Rosenbrock methods to the analysis of flexible multibody systems. Multibody System Dynamics, 10:263-288, 2003. 10.1023/A:1025990403660.

[42] J. P. Meijaard, R. G. K. M. Aarts, and D. M. Brouwer. Modelling and simulation of the motion of a slender beam in a tube. In The $2^{\text {nd }}$ Joint International Conference on Multibody System Dynamics, Stuttgart, Germany, May 29-June 12012.

[43] O. A. J. Meijden and M. P. Schijven. The value of haptic feedback in conventional and robot-assisted minimal invasive surgery and virtual reality training: a current review. Surgical Endoscopy, 23(6):1180-1190, 2009.

[44] Micro-Epsilon Messtechnik, 94496 Ortenburg, Germany. Laser Sensors for Displacement and Position, 2010.

[45] MISUMI Europa GmbH, Katharina-Paulus-Straße 6, D-65824 Schwalbach, Germany. Mechanical Components for Assembly Automation, 2010 .

[46] A. M. Okamura. Haptic feedback in robot-assisted minimally invasive surgery. Current Opinion in Urology, 19(1):102-107, January 2009.

[47] A. M. Okamura, C. Simone, and M. D. OLeary. Force modeling for needle insertion into soft tissue. In IEEE TRANSACTIONS ON BIOMEDICAL ENGINEERING, volume 51, OCTOBER 2004.

[48] Olympus America Inc. Endoscopy - A Backgrounder. http:// www.olympusamerica.com/presspass/press_pass_cut/msg_ landing. asp, May 2013.

[49] Olympus America Inc. Olympus endotherapy. http://www. olympusamerica.com/presspass/press_pass_cut/msg_ pressone.asp, May 2013.

[50] M. Rosen and J. Ponsky. Minimally invasive surgery. Endoscopy, 33(4):358-366, 2001.

[51] I. Rutkow. Origins of modern surgery. In J. Norton, P. Barie, R. Bollinger, A. Chang, S. Lowry, S. Mulvihill, H. Pass, and R. Thompson, editors, Surgery, pages 3-19. Springer New York, 2008. 
[52] P. Schellekens, N. Rosielle, H. Vermeulen, M. Vermeulen, S. Wetzels, and W. Pril. Design for precision: Current status and trends. CIRP Annals - Manufacturing Technology, 47(2):557 - 586, 1998.

[53] L. F. Shampine and M. K. Gordon. Computer solution of ordinary differential equations: the initial value problem. W. H. Freeman and Company, San Francisco, CA, 1975.

[54] A. H. Slocum. Precision Machine Design. Society of Manufacturing Engineers, Dearborn, Michigan 48121, 1992.

[55] H. M. J. R. Soemers. Design Principles for Precision Mechanisms. TPoint Print, Enschede, Netherlands, 2010. ISBN 978-90-365-3103-0.

[56] I. M. Stuart. Capstan equation for strings with rigidity. British Journal of Applied Physics, 12(10):559-562, 1961.

[57] L. L. Swanström, Y. Khajanchee, and M. A. Abbas. Natural Orifice Transluminal Endoscopic Surgery: The Future of Gastrointestinal Surgery. The Permanente Journal, 12(2):42-47, Spring 2008.

[58] L. L. Swanström, R. Kozarek, P. J. Pasricha, S. Gross, D. Birkett, P.-O. Park, V. Saadat, R. Ewers, and P. Swain. Development of a new access device for transgastric surgery. Journal of Gastrointestinal Surgery, 9(8):1129-1137, 2005.

[59] L. L. Swanström, M. Whiteford, and Y. Khajanchee. Developing essential tools to enable transgastric surgery. Surgical Endoscopy, 22(3):600604, 2008.

[60] H. Ten Hoff. Scanning Mechanisms for Intravascular Ultrasound Imaging: a flexible approach. PhD thesis, Erasmus Universiteit Rotterdam, the Netherlands, 1993.

[61] G. Tholey, J. P. Desai, and A. E. Castellanos. Force feedback plays a significant role in minimally invasive surgery: Results and analysis. Annals of Surgery, 241(1):102-109, January 2005.

[62] A. L. Trejos, R. V. Patel, and M. D. Naish. Force sensing and its application in minimally invasive surgery and therapy: A survey. Proceedings of the Institution of Mechanical Engineers, Part C: Journal of Mechanical Engineering Science, 224(7):1435-1454, July 12010. 
[63] K. v. d. Werff and J. B. Jonker. Dynamics of flexible mechanisms. In E. J. Haug, editor, Computer aided analysis and optimization of mechanical system dynamics, NATO ASI series. Series F ; vol. 9, pages 381-400. Springer-Verlag, 1984. 



\section{The Planar Beam Element}

Figure A.1 shows a planar beam element in an $x y$ inertial coordinate system. It consists of two nodes, $p$ and $q$, defined at the either end of the beam. The configuration of the beam element is described by the position vectors, $\boldsymbol{x}^{p}$ and $\boldsymbol{x}^{q}$, of the end nodes, $p$ and $q$, and the angular orientation of the orthonormal triads, $\left(\boldsymbol{n}_{x}, \boldsymbol{n}_{y}\right)$, rigidly attached to the element nodes [63]. The rotation part of the motion of the beam element is described by the planar rotation matrices $\boldsymbol{R}^{p}$ and $\boldsymbol{R}^{q}$, defined by

$$
\boldsymbol{R}^{p}=\left[\begin{array}{rr}
\cos \phi^{p} & -\sin \phi^{p} \\
\sin \phi^{p} & \cos \phi^{p}
\end{array}\right], \quad \boldsymbol{R}^{q}=\left[\begin{array}{rr}
\cos \phi^{q} & -\sin \phi^{q} \\
\sin \phi^{q} & \cos \phi^{q}
\end{array}\right] .
$$

The nodal coordinates of the beam element are four Cartesian coordinates $\left(x^{p}\right.$, $\left.y^{p}\right),\left(x^{q}, y^{q}\right)$ describing the position of the beam in the $(x, y)$-coordinate system and two rotation angles $\phi^{p}$ and $\phi^{q}$ representing the angular orientation of the base vectors $\left(\boldsymbol{R}^{p} \boldsymbol{n}_{x}, \boldsymbol{R}^{p} \boldsymbol{n}_{y}\right)$ and $\left(\boldsymbol{R}^{q} \boldsymbol{n}_{x}, \boldsymbol{R}^{q} \boldsymbol{n}_{y}\right)$ at the nodes $p$ and $q$ respectively. Hence the vector of nodal coordinates is given by

$$
\boldsymbol{x}_{\text {beam }}^{(k)}=\left[\begin{array}{lllllll}
x^{p}, & y^{p}, & \phi^{p} & \mid & x^{q}, & y^{q}, & \phi^{q}
\end{array}\right]^{T} .
$$

There are three deformation modes defined for the planar beam. The deformation $\epsilon_{1}^{(k)}$ represents the elongation of the element, and the other two deformation modes, $\epsilon_{2}^{(k)}$ and $\epsilon_{3}^{(k)}$, are associated with the flexible deformation of the beam element. They are defined as $[1,21,63]$ :

elongation:

$$
\begin{array}{rlrr}
\epsilon_{1}^{(k)} & =\mathcal{D}_{1}^{(k)}\left(\boldsymbol{x}^{(k)}\right) & = & l^{(k)}-l_{0}^{(k)}, \\
\epsilon_{2}^{(k)} & =\mathcal{D}_{2}^{(k)}\left(\boldsymbol{x}^{(k)}\right) & = & -\left(\boldsymbol{R}^{p} \boldsymbol{n}_{y}, \boldsymbol{l}^{(k)}\right), \\
\epsilon_{3}^{(k)} & =\mathcal{D}_{3}^{(k)}\left(\boldsymbol{x}^{(k)}\right) & = & \left(\boldsymbol{R}^{q} \boldsymbol{n}_{y}, \boldsymbol{l}^{(k)}\right),
\end{array}
$$




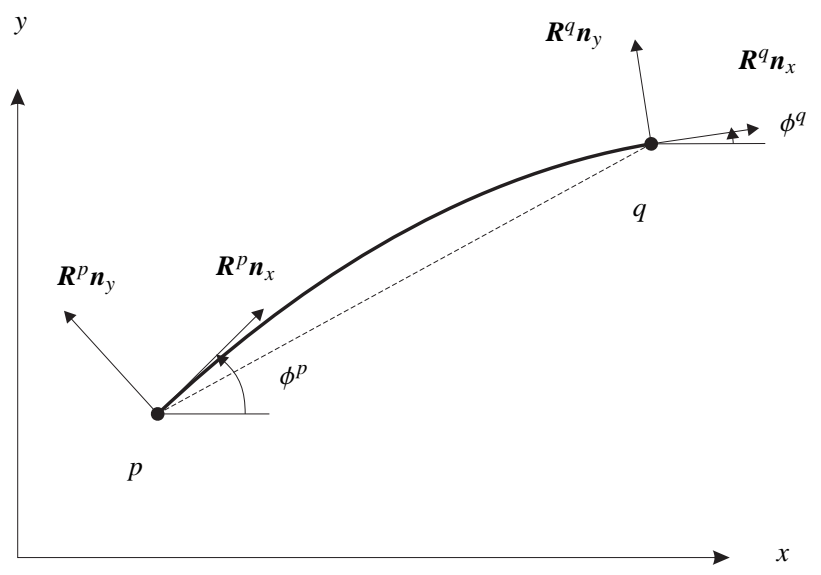

Fig. A.1: The planar flexible beam element

where $l_{0}^{(k)}$ is the reference length of the element, $l^{(k)}$ is the length of the vector $\boldsymbol{l}^{(k)}$, and the vector $\boldsymbol{l}^{(k)}$ is defined by

$$
\boldsymbol{l}^{(k)}=\boldsymbol{x}^{q}-\boldsymbol{x}^{p}=\left[x^{q}-x^{p}, y^{q}-y^{p}\right]^{T} .
$$

The operator $(\boldsymbol{a}, \boldsymbol{b})$ denotes the inner product of vectors $\boldsymbol{a}$ and $\boldsymbol{b}$. 


\section{SUMMARY}

Flexible instruments are increasingly used to perform advanced and complex surgical procedures either manually or robotically. The success of flexible instruments in surgery requires high motion and force fidelity, and controllability of the tip. However, the friction and the limited stiffness of such instruments limit the motion and force transmission of the instrument. The implementation of flexible instruments in surgery necessitates characterization of the flexible instruments, development of proper tools to understand the effect of various mechanical parameters on the overall performance, and an improvement in motion and force fidelity.

Therefore, the objective of this research is stated as: Characterization of static and dynamic behaviour of a flexible surgical instrument inside a curved rigid tube - both for translation and rotation motion input; develop a 3-D flexible multibody model; design a dedicated experimental set-up for the actuation and measurement of motion and interaction forces; and derive strategies to improve motion and force fidelity of the instrument. We developed a 2-D flexible multibody model and studied the sliding behaviour of the instrument with respect to the coefficient of friction and the bending stiffness of the instrument. Later, We developed a general 3-D flexible multibody model to study the static and dynamic behaviour of the instrument both in translation and rotation. The developed model is able to demonstrate the characteristic behaviour of the flexible instrument for a given set of parameters. The simulation results showed a deviation of $1.8 \%$ in the estimation of input force compared with the analytical capstan equation. The simulation results showed the motion hysteresis in translation and rotation. The onset of whipping motion in rotation is also observed due to a pre-curvature and a difference in the flexural rigidity along the principal axes of the instrument.

An experimental set-up is designed to provide the input motion both in translation and rotation from the proximal end, to measure the distal end motion without contact, and to measure the interaction forces between the instrument and the endoscope. The set-up is evaluated with respect to the design specifi- 
cations. The achieved specifications allow measurement of the characteristic behaviour of the instrument inside a curved rigid tube and the validation of the flexible multibody model. We performed various experiments to validate the developed model and also to characterize the instrument behaviour.

We derived strategies to improve motion and force fidelity of the instrument. The force fidelity is increased by combining the motion. Analytical model is presented and validated with the modelling and experimental results. A reduction in the friction force increases the force fidelity of the instrument. 


\section{ACKNOWLEDGEMENTS}

The years—spent during this $\mathrm{PhD}$ research - have been incredible and magnificent. I have accomplished the biggest and the most earnest dream of my life. I would like to thank everyone who helped me directly or indirectly in my pursuit of higher learning and in achieving the highest accolade of higher education and scientific research.

Firstly, I would like to thank my mentors and supervisors-Dannis Brouwer, Ronald Aarts, Herman Soemers and Just Herder. Dannis and Herman, many thanks for entrusting me with the responsibility and giving me your full support and confidence in performing my research. I am privileged to learn the basics of precision machine design under your tutelage. Dannis, thanks for being my daily supervisor. We discussed heartily in almost every possible topics. You allowed me to explore new ideas, but also pulled me back whenever I was veering away from my main focus. Thanks for giving me an opportunity to teach some of your lectures. Ronald, I learned a lot from your expertise in modelling and control. Thanks for your insightful comments. Just, thanks for being my promoter. You took me under your supervision mid-way. I was hugely benefited from your knowledge and experience in managing $\mathrm{PhD}$ research. You quickly streamlined my research, and my thesis has started taking shape. Thanks for that extra push and encouragement.

Secondly, I would like to thank the members of my Graduation CommitteeProf. S. Mukherjee, Prof. P. Breedveld, Prof. S. Stramigioli and Prof. F. van Houten-for taking out your precious time to evaluate my thesis and travelling here for my defence. I express my gratitude for all your effort and kindness. I appreciate your kind words and valuable suggestions.

Furthermore, I would like to thank my colleagues from Mechanical Automation group. I would like to thank Prof. Ben Jonker for your immense contribution towards flexible multibody modelling. Ben, thanks for your inquisitiveness and your interest in my work. I was immensely benefited from your knowledge and expertise in multibody modelling. Jaap, I really appreciate your help and support in using SPACAR. Johannes, thanks for teaching me some basics of PID control and sharing some useful tips. Martina, thanks for your support for all the official work. I kept bothering you with the Dutch 
documents and you always helped me with the right information. You helped me quickly settle in the Netherlands with my family. Leo, thanks for your readiness to help. I really appreciate your contribution towards the realization of the set-up. I would like to thank Tjeerd and Steven for all the discussions and help related to modelling and SPACAR. Ger, thanks for your help in setting up the XPC target. Thanks to Wouter, Dirk, Daniel, Bert, Johann, Paul, Volkert, Niya and Michiel for lively discussions on various topics during the coffee breaks. Thanks for the pleasant time we had in the office and outside. Daniel, thanks for being one of my paranymphs. Marieke, I would like to thank you for your contribution towards 2-D flexible multibody modelling and simulation, and wish you great success in your career.

I would like to thank my colleagues from the TeleFLEX project. Rob, Martin, Jeroen and Michel, thanks for your company. We visited most of the hospitals in the Netherlands together and interviewed the members of the project user-group. It was a great learning experience from me. Stefano and Ivo, thanks for your enthusiasm and vision for surgical robotics.

I would like to thank my Indian friends here at UT. I really appreciate ISA in bringing Indian people together and organizing various activities. I appreciate AADHAAR for doing a great job in contributing to the society by doing several projects in rural India. Pramod, thanks for introducing me and entrusting me the responsibility. I am successfully running a knowledge centre in my village. Special thanks to-Vishakha-Pramod, Meenakshi-Chandu, SangeetaVijay, Aabha-Digvijay, Chetna-Amrish, Ruchi-Jai, Pallavi-Pandu, ChaithanyaShodhan, Gayathri-Ganesh, Sampada-Omkar, Ann-Ravi, Shruti-Abhinendra, Sablika-Himanshu, Priyanki-Ramen, Neeru-Hanumant and Nupur-for all the little joy we shared together. Hanumant Singh, thanks for being a good friend and for agreeing to be my paranymph. I would also like to thank the badminton club DIOK for having great time. I visited many badminton clubs in the region and thoroughly enjoyed it.

Finally, I would like to thank my parents, brothers, sisters, and my relatives in India for all the love and encouragement. I really miss you all. I express my love to my wife Neelam for your wholehearted and unwavering support. My little daughter Bhairavi, you are the inspiration and the real joy of our life. You made my stay pleasant and full of surprises.

Above all, I wish to remember and pay my obeisance and reverence to The Almighty for His graciousness. Thanks for everything! 


\section{About the Author}

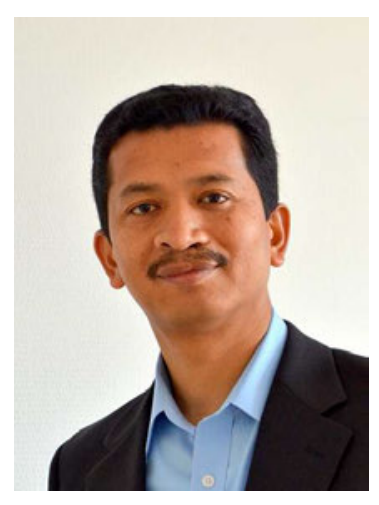

Jitendra Prasad Khatait was born on September 21, 1977 in a small village, Narayan Garh, of West Champaran district in Bihar, INDIA. He did his primary schooling at the village school. He got admission to Navodaya Vidyalaya in 1986 for secondary and senior secondary education through a district-wide competition. He spent next 7 years there under the guidance of his teachers.

Jitendra received his Bachelor of Engineering degree in Mechanical Engineering from Indian Institute of Technology Roorkee (then University of Roorkee), India (1996-2000). He graduated with First division with Honours. He was awarded with Chancellor's Gold Medal. He worked briefly as a Mechanical Engineer in Daewoo Motors, India (2000-2001).

He continued his further education and received his Master of Technology degree in Mechanical Design from Indian Institute of Technology Delhi, India (2001-2002). He was awarded with Padmashri Manmohan Suri Project Award for the best innovative project.

To further enhance his knowledge on Mechatronics, he went to Singapore to pursue Master of Technological Design program in Mechatronics jointly by National University of Singapore and Eindhoven University of Technology, Netherlands (2003-2005). He did his research internship in Singapore Institute of Manufacturing Technology, A-STAR, Singapore. Later, he continued working there as a Research Engineer in Mechatronics group (2005-2008). He worked on several projects related to precision machine design. He worked extensively with the air bearings.

Finally, he decided to move to Enschede, Netherlands to pursue his Ph.D. research at University of Twente, Netherlands (2009-2013). His research focus was on mechanical design of surgical robotic system using a flexible endoscope and instruments. 
His research interests are in precision machine design, medical devices, flexible surgical instrument, and medical robotics.

His other interests include painting, running, playing sports, etc. He is running a knowledge centre in his village to provide a good learning environment to the village kids. 

Flexible instruments are increasingly used to perform advanced and complex surgical procedures either manually or robotically. The success of flexible instruments in surgery requires high motion and force fidelity, and controllability of the tip. However, the friction and the limited stiffness of such instruments limit their motion and force transmission. The implementation of flexible instruments in surgery necessitates their characterization, the development of proper tools to understand the effect of various mechanical parameters on their overall performance, and an improvement in motion and force fidelity. Therefore, this thesis describes 2-D and 3-D flexible multibody models of a flexible surgical instrument inside a curved endoscope, and covers the design and evaluation of a dedicated experimental set-up. The thesis also includes the evaluation of the models with the analytical and experimental results, and describes the strategy to improve force transmission along the axial direction due to the combined motion input at the proximal end of the instrument.
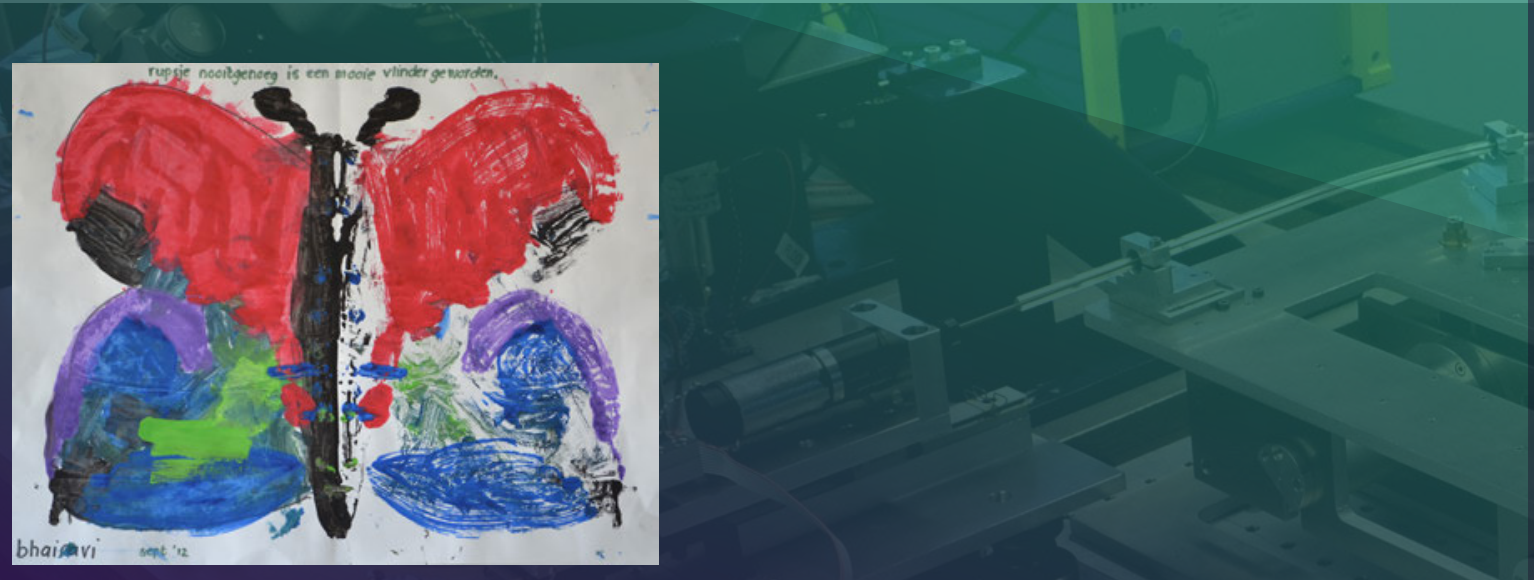